\title{
STED Microscopy with Scanning Fields Below the Diffraction Limit
}

\author{
Dissertation for the award of the degree \\ "Doctor rerum naturalium" \\ within the doctoral program IMPRS-PBCS \\ of the Georg-August University School of Science (GAUSS)
}

submitted by

Fabian Göttfert

born in Zürich, 14.12.1986

Göttingen, 2015 


\section{Thesis Committee}

Stefan Hell, Department of NanoBiophotonics, Max Planck Institute for Biophysical Chemistry, Göttingen

Sarah Köster, Institute for X-Ray Physics / Nanoscale Imaging of Cellular Dynamics, Georg-August-Universität Göttingen

Erwin Neher, Professor emeritus, Max Planck Institute for Biophysical Chemistry

\section{Members of the Examination Board}

Referee: $\quad$ Stefan Hell

2nd Referee: Sarah Köster

\section{Further members of the Examination Board}

Erwin Neher

Alex Egner, Optical Nanoscopy, Laser-Laboratory Göttingen

Katrin Willig, Nanoscale Microscopy and Molecular Physiology of the Brain, Max Planck Institute of Experimental Medicine, Göttingen

Tobias Moser, Institute for Auditory Neuroscience, University Medical Center Göttingen

Oral examination: 01.12.2015 


\begin{abstract}
Since its development, STED microscopy has been used extensively for imaging biological samples. To label structures of interest, fluorescent proteins and organic dyes are used almost exclusively. However, these labels undergo irreversible photobleaching reactions when illuminated, which limits the available signal and ultimately the feasible resolution.

This thesis presents a new approach to increase the signal yield in STED microscopy and related techniques. In these methods, the area that contributes to the fluorescence signal is much smaller than the illuminating laser foci. Thus, the irradiation dose per dye molecule can be significantly reduced by confining the image size to below the illuminated area. The probability of a photobleaching event during one image frame is reduced and more signal can be acquired.
\end{abstract}

A STED microscope specialized for small scan areas was built. As only few sample points are imaged, the frame acquisition time is in the order of ten milliseconds. High scan frequencies would lead to oscillations in mechanical beam scanners. Therefore, it was necessary to use electro-optic beam deflectors to guarantee accurate positioning of the laser foci. A software was developed to select the desired scan-area positions from an overview image, which is acquired with a piezo-scanning stage. The viability of this approach was demonstrated on a variety of samples. It was shown on the dye Atto647N that the bleaching rate scales approximately linear with the STED intensity. Thus, the profit gained by reducing the scan area increases with the used STED laser power. In the limit of zero STED power, the photon yield of a fluorophore would be unchanged. Furthermore, the structural details in the focus area would not be resolvable. When increasing the resolution with STED to $20 \mathrm{~nm}$, however, the reduction of the scan area to $70 \times 70 \mathrm{~nm}^{2}$ yields 100 times more signal as compared to a regular scan size. This makes the developed method especially useful for investigating structures smaller than 200 nanometers. As an example, the nucleoporins NUP98 and NUP93 were imaged at a resolution below $20 \mathrm{~nm}$, revealing their arrangement in the nuclear pore. 



\section{Contents}

1. Introduction 1

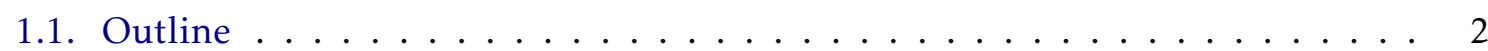

1.2. Optical Microscopy . . . . . . . . . . . . . . . . . . 3

1.2.1. Image Formation . . . . . . . . . . . . . 3

1.2.2. Extending the Diffraction Barrier . . . . . . . . . . . . . . 4

1.2.3. Breaking the Diffraction Barrier . . . . . . . . . . . . 5

1.3. STED Microscopy . . . . . . . . . . . . . . . . . . . . . 7

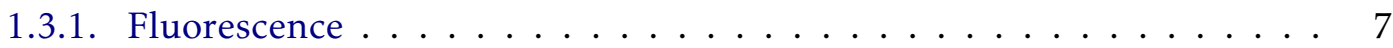

1.3.2. Stimulated Emission . . . . . . . . . . . . . . . . . 9

1.3.3. Breaking the Diffraction Barrier with STED . . . . . . . . . . . . 9

1.4. Motivation . . . . . . . . . . . . . . . . . . 11

1.4.1. Two-Color STED . . . . . . . . . . . . . . . . . . 11

1.4.2. Scanning a Field of View Below the Diffraction Limit . . . . . . . . . . . 12

2. Methods 15

3. Two-Color STED Microscopy 17

3.1. Setup Design . . . . . . . . . . . . . . . . . 17

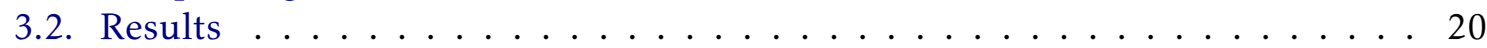

3.2.1. Channel Crosstalk . . . . . . . . . . . . . . . . 20

3.2.2. Co-localization Accuracy . . . . . . . . . . . . . . . . . . 21

3.2.3. Resolution . . . . . . . . . . . . . . . . . 22

3.2.4. Flourescence Correlation Spectroscopy (FCS) . . . . . . . . . . . . . 22

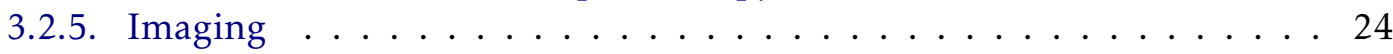

4. Scanning Field of Views Below the Diffraction Limit 27

4.1. Design of the Experiment . . . . . . . . . . . . . . . . . 27

4.1.1. Beam Scanning . . . . . . . . . . . . . . . . . 27

4.1.2. Electro-Optic Deflectors . . . . . . . . . . . . . . . 28

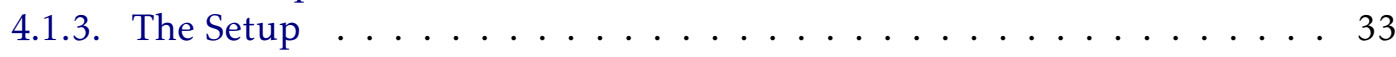

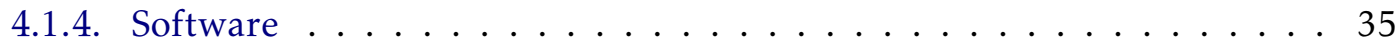

4.2. Bleaching in STED Microscopy . . . . . . . . . . . . . . . . 41

4.2.1. Considerations . . . . . . . . . . . . . . . . . 41

4.2.2. Modeling the Bleaching Behavior . . . . . . . . . . . . . . . 42

4.2.3. Analysis of the Bleaching Data . . . . . . . . . . . . . . 45

4.2.4. Fitting the Model . . . . . . . . . . . . . . . . . . . 46

4.2.5. Application to STED Measurements . . . . . . . . . . . . . . 50

4.3. Application Examples . . . . . . . . . . . . . . . . . . 52

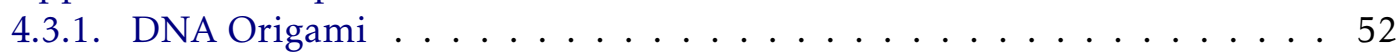

4.3.2. Nanobodies . . . . . . . . . . . . . . . . . . . 55 


\section{Contents}

5. Discussion $\quad 59$

$\begin{array}{ll}\text { Bibliography } & 65\end{array}$

A. Appendix $\mathbf{7 3}$

A.1. Resolution of the Two-Color Setup . . . . . . . . . . . . . 73

A.2. DNA Origami . . . . . . . . . . . . . . . 74

A.3. Single Molecule Bleaching . . . . . . . . . . . . . . 76

A.4. Nanobodies . . . . . . . . . . . . . . . . . . . . . 82

A.5. Published Articles . . . . . . . . . . . . . . . . . . . . 83 


\section{List of Abbreviations}

\begin{tabular}{|c|c|}
\hline $\mathrm{APD}$ & avalanche photo diode \\
\hline a.u. & arbitrary units \\
\hline CL & cylinder lens \\
\hline $\mathrm{CW}$ & continuous wave \\
\hline FCS & fluorescence correlation spectroscopy \\
\hline FOV & field of view \\
\hline FWHM & full width at half maximum \\
\hline ISC & intersystem crossing \\
\hline NA & numerical aperture \\
\hline NPC & nuclear pore complex \\
\hline NUP & nucleoporin \\
\hline PBS & phosphate-buffered saline \\
\hline PSF & point spread function \\
\hline RESOLFT & reversible saturable/switchable optical fluorescence transitions \\
\hline SNR & signal-to-noise ratio \\
\hline STED & stimulated emission depletion \\
\hline TL & telescope lens \\
\hline
\end{tabular}





\section{Introduction}

The history of scientific discoveries is closely interweaved with the development of new tools to observe and manipulate our environment. This relationship is especially noticeable in the development of optics: Today light, or rather electromagnetic radiation, is used in almost all fields of science as a tool and sampling method. Although mirrors and the ray properties of light have been known for millennia, the oldest written record of a lens is only 1000 years old [1]. It took several centuries until the invention of the microscope and telescope in the late 16th century. What then followed was an unprecedented series of scientific advances in astronomy and biology. Every increase in the quality of the lenses and lens systems led to new discoveries. Until the middle of the 19th century, lenses were produced by trial and error without any fundamental understanding of their working principle. In the late $1860 \mathrm{~s}$ Carl Zeiss asked Ernst Abbe to develop a theory of microscopic imaging [2]. One of his most fundamental discoveries was that the resolution of an imaging system is limited by the diffraction of light, known as the diffraction limit:

$$
d=\frac{\lambda}{2 n \sin \alpha}
$$

This formula states that two small objects separated by a distance smaller than $d$ cannot be identified as individual objects by a microscope operating with light at wavelength $\lambda$ and a lens with the so called numerical aperture NA $=n \sin \alpha$, where $n$ is the refractive index of the sample medium and $\alpha$ the half-opening angle of the objective (Figure 1.1). Abbe's theory provided the insight necessary to build microscopes better than ever before, soon approaching the diffraction limit.

There exist other versions of the resolution limit, differing from (1.1) by a constant factor, such as the Rayleigh criterion or Sparrow's resolution limit, estimating the minimal distance at which two objects can still be separated. To a certain degree this minimal distance is dependent on the application and the object's shape [3]. With the above equation, the resolution of a standard light microscope is limited to $>160 \mathrm{~nm}$ when operating at $500 \mathrm{~nm}$ wavelength and using an immersion oil objective with a numerical aperture of 1.4. Methods extending the diffraction barrier are discussed in section 1.2.2.

The diffraction barrier is a physical limit for any imaging system that relies on focusing waves. Several techniques employ the shorter wavelengths of X-ray or electron beams to achieve atomic-scale resolution [4]. Other imaging systems use small probes in close proximity to the sample to either avoid the diffraction by measuring the optical near field [5] or to measure other parameters like the force acting on the probe [6] and density of electronic states [7].

Although these methods can provide resolution down to the atomic scale, far-field light microscopy is still widely used. One reason certainly is the comparatively simple setup and easy sample preparation for a standard light microscope. For many applications, especially when investigating biological structures, the light microscope offers possibilities that are denied by other methods. The minimally invasive nature of light allows imaging inside living 


\section{INTRODUCTION}

organisms without disturbing their environment. In fluorescence microscopy, proteins are tagged with specific fluorescent labels to study their organization and interaction in space and time with very high contrast. Specialized fluorescence microscopy methods can measure the interaction of molecules at the nanometer scale [8] using Förster resonance energy transfer, and with microsecond time resolution by analyzing fluctuations in the fluorescence signal $[9,10]$.

Combining these advantages with diffraction-unlimited resolution has become possible with the development of Stimulated Emission Depletion (STED) microscopy by Stefan Hell, first proposed by Hell and Wichmann in 1994 [11]. It was the first far-field microscopy method to break the diffraction barrier, i.e. its resolution is not limited by the numerical aperture of the objective or the wavelength used. Based on fluorescence microscopy techniques, STED microscopy uses a laser to selectively switch off fluorescent molecules through stimulated emission. The attainable resolution in its most common implementation is given by [12]:

$$
d_{\mathrm{STED}}=\frac{d_{\mathrm{c}}}{\sqrt{1+d_{\mathrm{c}}^{2} a^{2} \frac{I}{I_{0}}}} \stackrel{\stackrel{I}{I_{0}} \gg 1 / d_{\mathrm{c}}^{2} a^{2}}{\longrightarrow} \frac{1}{a \sqrt{\frac{I}{I_{0}}}}
$$

where $d_{\mathrm{c}}$ is the diffraction-limited resolution of equation (1.1), $a$ is an implementationdependent factor with dimension $\left[\mathrm{m}^{-1}\right], I$ is the intensity of the laser inducing stimulated emission, measured at the maximum position of the intensity pattern, and $I_{0}$, often called saturation intensity, is a normalization factor for $I$ depending on the fluorescent dye and wavelength used. The resolution $d_{\text {STED }}$ approaches 0 for high laser intensities, yet is ultimately limited by the dye molecule's size. An experimental resolution $<3 \mathrm{~nm}$ has been reached in experiments with fluorescent defects in diamond [13]. In biological samples, a resolution below $20 \mathrm{~nm}$ is feasible. Above equation is described in more detail in section 1.3.3.

\subsection{Outline}

Chapter 1 will give a brief overview of the concepts involved in super-resolution microscopy, focusing on STED microscopy in particular. The thesis projects are introduced at the end of this chapter. The methods used for sample preparations are described in Chapter 2.

Within this thesis, two implementations of a STED microscope were realized: Chapter 3 presents a setup designed for two-color imaging with red and far red organic dyes. Chapter 4 is the main part of this thesis. A new concept to increase the signal yield in STED microscopy has been successfully realized: Confining the field of view to areas below the diffraction limit reduces the bleaching per acquired frame drastically and allows measurements with a previously unattainable level of detail.

The results are discussed in Chapter 5, concluding with an outlook for future applications and improvements. 


\subsection{Optical Microscopy}

\subsubsection{Image Formation}

Far field microscopes use lenses to produce a virtual or real image of a sample, which is then observed by the eye or a detector. While it is possible to use a single lens to produce a magnified image, modern microscopes use lens systems. Figure 1.1 depicts the basic operating principle of a microscope using a camera as detector and an infinity-corrected objective: The sample is located in the objective's focal plane. It may be illuminated through the objective or by other means. The light signal is collected and collimated by the objective lens, then focused by the projection lens onto the detector. The magnification $m$ of the microscope is given by the relation of the focal lengths $f$ of the objective and projection lenses:

$$
m=\frac{f_{\mathrm{P}}}{f_{\mathrm{O}}}
$$

However, since light propagates as a wave, it is diffracted by the aperture of the objective and projection lenses, resulting in a blurred image. The diffraction at circular apertures was first derived by Airy [14] in 1835 for telescopes. The image of a point light source in the sample plane will be a so called Airy pattern, as depicted in Figure 1.1b. The intensity exhibits a central maximum, then drops to zero and forms smaller side maxima. The area surrounded by the first intensity zero is called Airy disk. Its diameter is

$$
d_{\text {Airy }}=1.22 \frac{\lambda}{n \sin \alpha}
$$

where $\lambda$ is the light wavelength, $n$ the refractive index of the sample medium, and $\alpha$ half

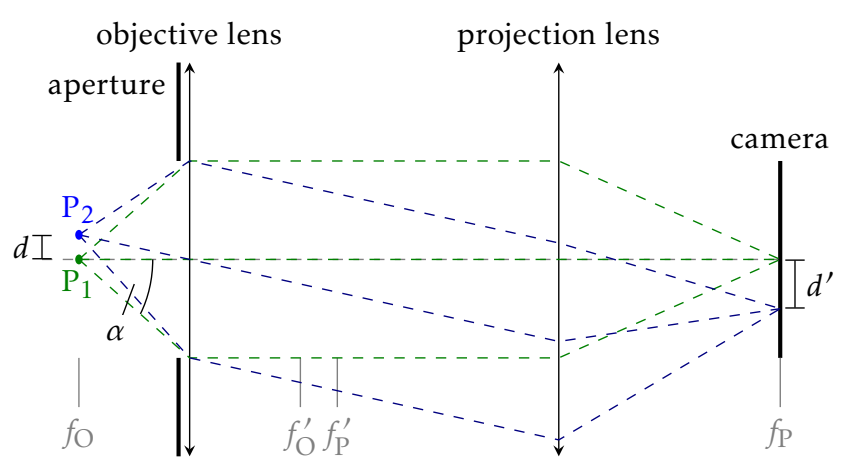

(a)

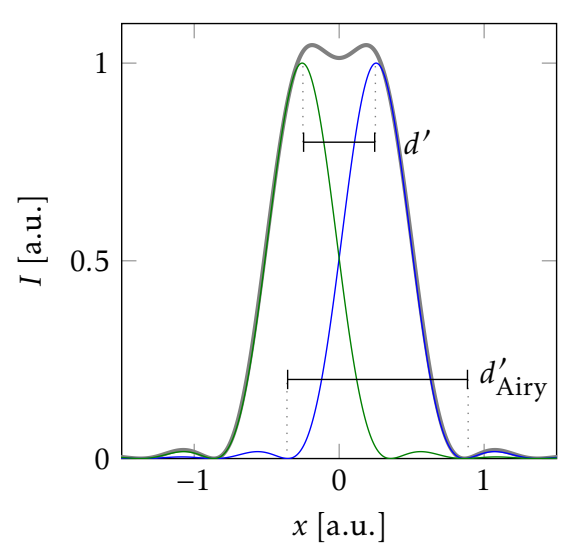

(b)

Figure 1.1. (a): Working principle of a wide field microscope with infinity-corrected objective. The sample points $\mathrm{P}_{1}$ and $\mathrm{P}_{2}$ in the objective's focal plane are imaged onto a camera by the objective and projection lenses. The magnification is $d^{\prime} / d=f_{\mathrm{P}} / f_{\mathrm{O}}$. Due to diffraction, a sample point is imaged as intensity distribution (b). The minimal width of the distribution is limited by the wavelengths used for imaging, the half opening angle $\alpha$ of the objective and the refractive index $n$ of the sample medium (equation (1.3)). When the light emitted by the sample is incoherent, the measured intensity (gray line) is the sum of several point distributions (colored lines). If the sample points are too close they become indistinguishable in the image. 


\section{INTRODUCTION}

the opening angle of the objective. The size of the Airy disk on the detector scales with the magnification $m$ of the microscope.

Two sample points are imaged as two Airy patterns. The more the images overlap, the harder it is to detect them as separate sample points. The Rayleigh criterion is an empirical threshold, stating that two points can be separated when the distance between their intensity maxima in the image is larger than the radius of the Airy disk. Since the Airy pattern is inconvenient to handle mathematically and the size of the Airy disk is hard to measure experimentally, the central intensity maximum is usually approximated as a Gaussian distribution of similar height and full width at half maximum (FWHM). The FWHM $=0.51 \lambda / n \sin \alpha$ serves as a useful, easy to measure resolution criterion. Figure 1.1b shows the calculated intensity distribution in the image of two points separated by the FWHM.

\section{The Point Spread Function}

Airy's diffraction pattern and Abbe's resolution limit model a microscope based on ideal lenses. Real systems today employ complex combinations of lens types and materials to reduce aberrations from the ideal to a minimum. One of the most general properties that characterize the performance of a microscope is the point spread function (PSF). The PSF is the image of an idealized point in the sample. In case of fluorescence microscopy, the PSF can be measured on a single isolated fluorophore or small ( $\ll$ resolution) labeled structure. The PSF includes all aberrations inherent to the imaging system and would be the Airy pattern for an ideal microscope.

The knowledge of the PSF is important when analyzing images, as it provides information on possible imaging artifacts. As the image is the sample structure convolved with the PSF, the PSF can be used to estimate the sample structure using deconvolution algorithms on the image [15].

In super-resolution microscopy the PSF is one of the most important measures of the imaging performance. Its size is no longer limited by diffraction and provides a good approximation for the resolution. However, the PSF cannot account for aberrations caused by the sample itself. Imaging heterogeneous samples such as tissue can lower the performance of any microscope drastically.

\subsubsection{Extending the Diffraction Barrier}

Confocal Microscope: Most STED setups are based on a confocal microscope. Instead of a camera acquiring a two-dimensional image, a pinhole is installed in the image plane and the transmitted light is detected by a single detector. The sample area that is imaged on the pinhole is illuminated with a focused laser beam. An image is acquired by scanning either the sample or the beam path and mapping the resulting signal to their position. When scanning the sample, the signal is proportional to the product of the illumination and detection function. In its ideal case for diffraction-limited illumination and a pinhole much smaller than the Airy disk, the image PSF is narrowed by a factor up to $\sqrt{2}$, thus increasing the resolution. However, this limit is difficult to achieve in practice [16]. The biggest advantage of confocal microscopy today is its sectioning capability in the z-direction: The focused illumination and spatially filtered detection strongly reduce the signal from out-of-focus planes, yielding an axial resolution of typically $>500 \mathrm{~nm}$. In contrast, the whole sample is illuminated in conventional camera based microscopes and out-of-focus signal leads to a strong 
blurred background in the image.

Increasing the NA: The numerical aperture $n \sin \alpha$ of the objective is the other defining figure limiting the resolution, next to the wavelength. The opening angle of present oil immersion objectives is $\approx 70^{\circ}$, therefore $\sin \alpha \approx 0.94$. Thus, better objectives will not improve the resolution significantly. The refractive index $n$ of the immersion and mounting medium has to be matched to the objective lens, otherwise aberrations decrease the imaging performance. The numerical aperture of present objective lenses is practically limited to $<1.5$.

However, the NA can be increased further by utilizing two opposing objectives to illuminate the sample or detect signal. The objectives are used as interferometers, resulting in a considerably higher axial resolution of better than $100 \mathrm{~nm}$. As the sample needs to be transparent, these techniques are solely used in fluorescence microscopy. This principle has been implemented in 4Pi microscopy [17] and $\mathrm{I}^{5} \mathrm{M}[18]$.

Increasing the Frequency Space: In case of a two-dimensional sample in the focal plane, the light field in the objective's back focal plane is the Fourier transformation of the sample signal. The aperture of the lens acts as a low pass filter for the spatial frequencies, thus limiting the resolution. The available frequency space can be extended by illuminating the sample with a structured pattern. By shifting its orientation, one can increase the accessible frequencies in the Fourier space by a factor of two, resulting in doubled resolution [19]. The frequencies of the illumination pattern are again limited by the wavelength and diffraction. However, the resolution can be further improved by exploiting a nonlinear response of the sample, thus increasing the spacial frequencies introduced by the illumination pattern [20, 21].

Deconvolution Prior knowledge of the imaging system and the sample can be used to estimate the sample structure from the blurred image. With sufficient information, structural features much smaller than the actual resolution may be identified [22]. In fluorescence microscopy, deconvolution is especially helpful to remove imaging artifacts [23].

\subsubsection{Breaking the Diffraction Barrier}

The methods described above all extend the resolution beyond Abbe's theoretical limit. However, with the exception of nonlinear structured illumination, their resolution is again limited by the objective's NA and the wavelengths used.

In contrast, super-resolution methods theoretically provide molecular resolution. They avoid the diffraction limit by precisely localizing the origin of the measured signal. Today, two concepts for far field super-resolution exist and are applied successfully in biological imaging:

- Targeted super-resolution methods aim to predefine the sampling area to a size below the diffraction limit. Reversible saturable/switchable optical (fluorescence) transitions (RESOLFT) are the core mechanisms to distinguish between signal emitted inside and outside the targeted area [24].

In the context of this thesis, the transition of the fluorophore from the bright $S_{1}$ to the dark $S_{0}$ state is induced by stimulated emission. A laser with a 'doughnut' focus profile is used to switch off excited fluorescent molecules (see Figure 1.5 and Figure 1.6b of the 


\section{INTRODUCTION}

next section). As the central intensity of the doughnut is zero, the fluorophores in the vicinity of the center will remain unperturbed. The measured signal originates only from the narrow, sub-diffraction sized central region.

The image is acquired by scanning the sampling area and measuring the signal strength for each position. The resolution is limited by the applicable power of the localization laser.

- Stochastic super-resolution methods localize single molecules by imaging them individually and calculating their position by fitting the image with the PSF. In order to image only one molecule per Airy unit at a time, most fluorophores are kept in a dark, non fluorescent state. Individual molecules switch to the bright fluorescent state stochastically, are localized and transit to a dark state $[25,26,27]$. The image acquisition period needs to be long enough to ensure most of the molecules have switched to the bright state at least once.

The image is reconstructed from the positions measured for each fluorophore. The resolution is limited by the number of photons detected per molecule.

Both concepts rely on a switching mechanism in the fluorescent probe that allows to separate two molecules in close proximity, yielding in principle molecular resolution. Fluorophores with high photostability are required, to acquire enough signal in the stochastic methods and to survive many switching cycles in the targeted approach. As super-resolution is a relatively recent development, current research includes the development of the fluorescent dyes, labeling techniques, microscopy techniques and their applications. 


\subsection{STED Microscopy}

Stimulated Emission Depletion (STED) microscopy exploits the properties of fluorescent molecules to increase the imaging resolution theoretically to a molecular level. This section introduces the mechanisms of fluorescence, stimulated emission and how they are utilized in STED microscopy.

\subsubsection{Fluorescence}

Fluorescence is the emission of light by a substance following excitation by irradiation. It occurs when the electronic structure of the material exhibits distinct energy levels: Electrons are excited to a higher state by absorbing a photon and emit a photon upon relaxation to the lower states.

In fluorescence microscopy, the fluorophore is usually an organic molecule or a fluorescent protein: The electronic wave functions of the individual atoms partly overlap, leading to a so-called $\pi$-bond where the electrons are delocalized over several atoms. The pattern of the potential energy and the confinement of the electron wave function to the molecule causes the formation of discrete electronic states. Vibrational and rotational states of the molecule affect the energy of the electronic states, leading to a broadening of the states into bands.

An electron in the ground state $S_{0}$ can generally be excited to any vibrational level of the excited state, provided that the energy difference matches the photon's energy. After excitation, the molecule will relax to the lower vibrational levels within a few picoseconds, returning to a thermal equilibrium with its environment. The excited electron can relax to the ground state by emitting a fluorescence photon, or radiationless via internal conversion. The probability of a radiative decay after excitation is called quantum yield. The lifetime of the excited electronic state is in the order of $1 \mathrm{~ns}$. The absorption and emission probabilities for photons with a certain energy follow the Franck-Condon principle, leading to the shape of the long decay of the excitation and emission spectra (Figure 1.2).

As energy is lost during the fluorescence cycle through the molecule's vibrational relaxation, the emitted photons usually have a lower energy than the excitation photons (Stokes Shift). This property is used in fluorescence microscopy to gain high contrast images of the labeled structure by blocking the excitation wavelength before detecting the signal.

Figure 1.2 shows the structure and spectrum of Star Red, a dye often used in the course of this thesis. The details of fluorescence photophysics can be better described when modeling the transitions in a Jablonski diagram (Figure 1.3): In addition to the singlet electronic states,
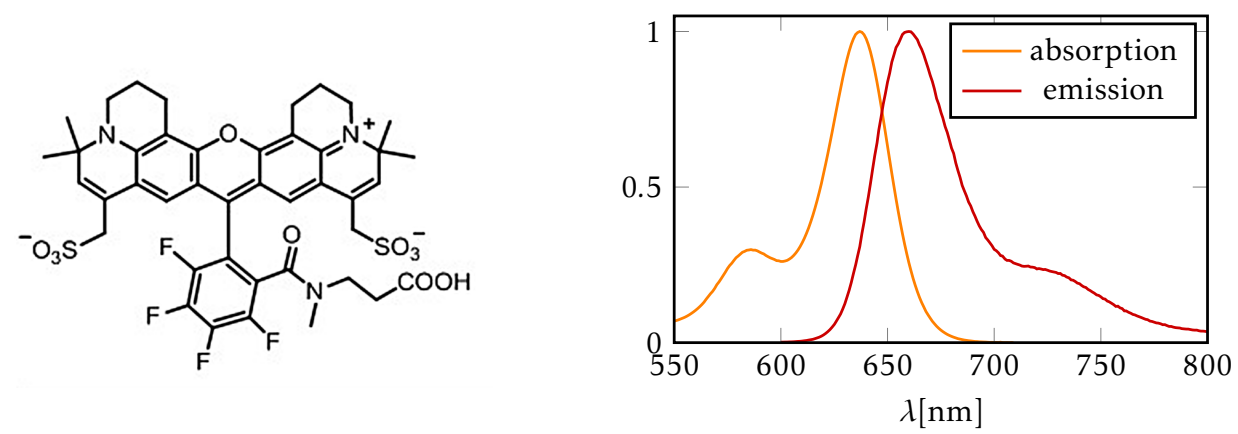

Figure 1.2. Structure [28] and spectrum of Star Red, a dye often used in STED microscopy. 


\section{INTRODUCTION}

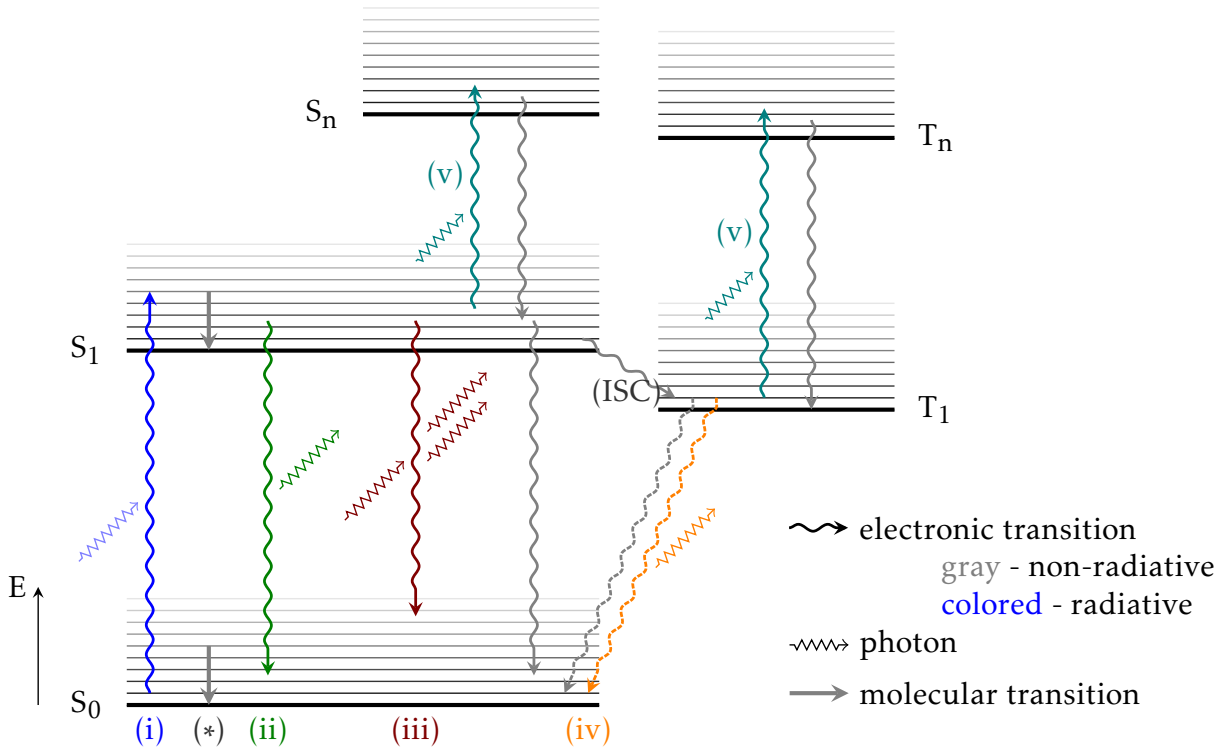

Figure 1.3. Jablonski diagram of a fluorescent molecule. The valence electrons of the molecule form discrete energy levels S (singlet) and T (triplet). At room temperature, vibrational and rotational states of the molecule broaden the levels into quasi continuous energy bands, leading to broad excitation and emission spectra. Transitions between states may be radiative, involving absorption or emission of a photon, or non-radiative through internal conversion. In the equilibrium at room temperature, the electrons are in the ground state $\mathrm{S}_{0}$. They can be excited to higher singlet states by absorbing an incoming photon and may transition to the triplet state by intersystem crossing (ISC).

The most important radiative transitions are: (i): Excitation from the ground state $S_{0}$ to $S_{1}$ through absorption of a photon; (ii): Spontaneous emission of a photon and transition $\mathrm{S}_{1} \rightarrow$ $\mathrm{S}_{0}$ (fluorescence); (iii): Stimulated emission of a photon and transition $\mathrm{S}_{1} \rightarrow \mathrm{S}_{0}$ induced by an incoming photon; (iv): Spontaneous emission of a photon and transition $\mathrm{T}_{1} \rightarrow \mathrm{S}_{0}$ (phosphorescence); (v): Excitation to higher states by absorption of a photon.

Only transitions considered in the model of section 4.2.2 are included in this figure. See main text for details.

triplet states exist in almost all dyes. Transitions between singlet and triplet states are called intersystem crossing (ISC) and occur through a spin flip of an electron, so the system has a total electronic spin of 1 and multiplicity 3 . These transitions are symmetry forbidden, yet strong spin-orbit coupling of the electrons can yield hight triplet quantum yields above 0.5 [29]. The triplet state is usually undesired and most of the used dyes have a yield $\ll 1 \%$. As the triplet state is usually energetically lower than the singlet, the reverse transition $T_{i} \rightarrow S_{i}$ is improbable, yet occurs for some dyes [30]. As the transition $\mathrm{T} \rightarrow \mathrm{S}$ is symmetry forbidden, the lifetime of the $T_{1}$ is considerably longer than that of the singlet, depending strongly on the environmental conditions. In solution it is typically several microseconds.

When the electron is excited to higher states $S_{n}$ and $T_{n}$, the relaxation is usually fast ( $\left.\ll 1 \mathrm{~ns}\right)$ and non-radiative (Kasha's rule [31]).

The complex mechanism of fluorescence and the direct exposure of the fluorophore to the environment can lead to a strong dependency of the fluorescent properties (e.g. lifetime, spectrum, quantum yield) to the environment. This can be utilized to sense local conditions, for example the $\mathrm{pH}$ or calcium concentration [32]. 
Alas, fluorescent molecules are also prone to photobleaching - the irreversible loss of fluorescence induced by irradiation. The mechanisms vary greatly between dyes and are extremely sensitive to the molecular environment and hard to determine experimentally. Photobleaching is discussed in more detail in Section 5.

\subsubsection{Stimulated Emission}

Stimulated emission is a fundamental property of the interaction of electrons with electromagnetic fields, in addition to absorption and spontaneous emission of a photon. An electron in the excited state can be stimulated to emit a photon by an incoming photon, thus loosing energy. The emitted photon has the same properties (wavelength, phase, momentum, polarization) as the photon inducing the emission, which is not affected by the process. A condition for stimulated emission is the existence of the lower electronic energy level.

In STED microscopy, the wavelength used for stimulated emission is red-shifted with respect to the absorption spectrum to avoid undesired excitation. Considering a dye population of $N$ excited molecules, the rate of stimulated emission is:

$$
\frac{\mathrm{d} N}{\mathrm{~d} t}=-\sigma_{\mathrm{stim}} I N
$$

where $I$ is the intensity of stimulating radiation and $\sigma_{\text {stim }}$ the cross-section for stimulated emission. $\sigma_{\text {stim }}$ primarily depends on the material and wavelengths used.

Therefore, the number of excited dye molecules decreases exponentially:

$$
N(t)=N\left(t_{0}\right) e^{-\left(\sigma_{\text {stim }} I t\right)}
$$

\subsubsection{Breaking the Diffraction Barrier with STED}

The steps to increase the resolution of a laser scanning fluorescence microscope with stimulated emission depletion are outlined in Figure 1.5. Here, only the implementation relevant for this thesis is discussed. Variants include the use of CW lasers [33] or parallelized excitation and STED [34], but they all rely on the same principles.

Typically, two laser beams are necessary to realize a STED microscope: The excitation laser is focused on to the sample and excites the fluorophores in a diffraction-limited area. The STED laser is focused on the same position and it forces the excited fluorophores to the ground state by stimulated emission.

Excitation of electrons from the ground state by the STED laser is negligible, as the equilibrium population of the high vibrational states is low and the STED photon energy is not sufficient to excite molecules from the lower vibrational states (see also Figure 1.3). It is possible that electrons de-excited by stimulated emission are re-excited by the STED laser before the molecule relaxes to the lower states. However, the vibrational relaxation takes place within $<1$ ps and high intensities would be required for considerable re-excitation. Pulsed lasers are more efficient for STED, as the fluorophores are first excited, then the STED laser quenches the fluorescence and the residual fluorescence signal is measured afterwards. Theoretically, a STED pulse length of $\approx 30 \mathrm{ps}$ is a good compromise between STED efficiency and avoiding re-excitation [35]. Longer pulses are usually used in practice, as laser sources with sufficiently short pulses are rather complex, the high peak intensities may induce two-photon absorption and the pulse synchronization on the picosecond scale 


\section{InTRODUCTION}

is technically challenging. In this thesis, the STED pulse length is fixed by the used laser to $1.2 \mathrm{~ns}$. As this is comparable to the fluorescence lifetime of the used dyes, time-gated detection is necessary to optimize the imaging performance (see Chapter 3 ).

Prior to entering the objective, the STED laser radiation passes a wavefront shaping device, leading to a specific intensity pattern in the focus. In this thesis a combination of a $2 \pi$ vortex phase plate and $\lambda / 4$ waveplate produces a 'doughnut' shaped focus with zero central intensity (Figures 1.4, 1.5 \& 1.6b ). The intensity distribution $I^{\prime}$ close to the center can be approximated with a parabola:

$$
I^{\prime}(r)=4 I a^{2} r^{2}
$$

where $I$ is the intensity at the maximum of the pattern, $a$ the steepness of the parabola and $r$ the distance from the center in the focal plane. The STED intensity rises quadratically with distance to the doughnut center and quenching of the fluorescence scales exponentially with the intensity, leaving only a small area of fluorophores close to the doughnut zero in the excited state. The resolution is defined as the full width at half maximum of the remaining fluorescent spot and depends on the applied STED laser power [12]:

$$
d_{\mathrm{STED}}=\frac{d_{\mathrm{c}}}{\sqrt{1+d_{\mathrm{c}}^{2} a^{2} \frac{I}{I_{0}}}}
$$

where $d_{c}$ is the FWHM of the diffraction-limited confocal spot and $I_{0}$ the STED intensity at which spontaneous and stimulated emission are equally probable. The above equation is valid for pulsed lasers with short pulses compared to the fluorescence lifetime. The resolution in the $z$-direction can be increased with different phase patterns [36].

The image is acquired by scanning the sample or laser beams and measuring the fluorescence for each position.
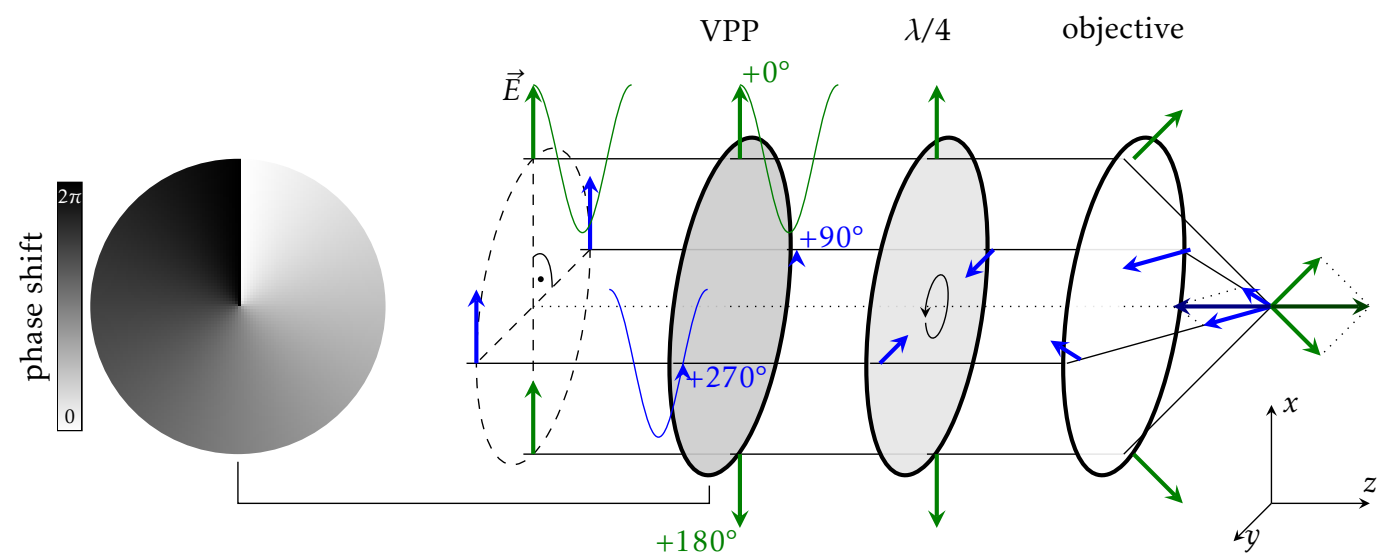

Figure 1.4. Creation of the central intensity zero in the STED 'doughnut'. The beam passes a vortex phase plate (VPP) which retards the beam by a phase of $0-2 \pi$, depending on the angular position. A $\lambda / 4$ waveplate circularizes the polarization. When the beam is focused, the components of the electromagnetic field cancel out. The colored arrows indicate the orientation and relative phase of the electric field vectors. 

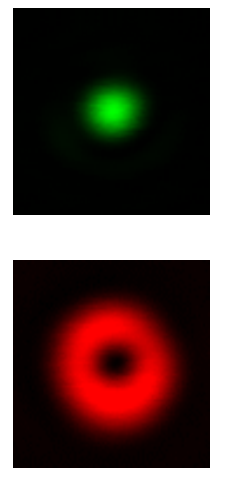

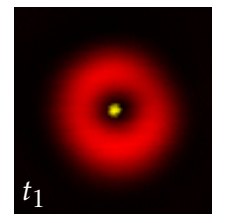

(iii)

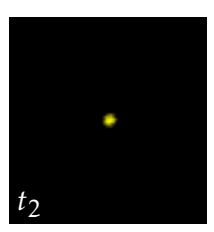

(ii) excitation laser

$\square$ excited molecules

STED laser

Figure 1.5. Steps leading to sub-diffraction resolution in STED microscopy: The molecules are excited with the excitation laser (i). The STED laser forces the excited molecules to the ground state by stimulated emission (iii). The remaining fluorescence (ii) originates from a sub-diffraction sized spot. It is collected by the objective and detected. The molecular processes for (i-iii) are outlined in Figure 1.3.

\subsection{Motivation}

\subsubsection{Two-Color STED}

Cellular processes rarely involve only one protein, there are a cascade of specialized molecules interacting with each other. It is therefore desirable to know their relative location, instead of investigating each one individually.

Several approaches exist to separate two structures labeled with different dyes. The first implementation of a two-color STED microscope used dyes with very little spectral overlap [37] and two pairs of STED and excitation lasers optimized for the respective dye. The biggest drawback of this approach is that the STED laser used for the blue-shifted dye will inevitably bleach the red-shifted dye, as it lies in its absorption spectrum. This cannot be avoided, as the absorption spectrum of fluorescent molecules extends far into bluer wavelengths. The imaging therefore needs to be frame-sequential and cannot be repeated for the red-shifted dye. Thermal drift and other error sources have to be minimized and compromise accurate co-alignment. A co-localization accuracy better than a few nanometer is experimentally not feasible when using multiple doughnuts [38, 39].

A solution to this problem is to use a second dye with a large Stokes shift and a similar emission spectrum [40], allowing to use a single STED laser. The color separation is achieved by using two excitation wavelengths, yet still requires unmixing of the image color channels, as both dyes are excited by the blue-shifted excitation laser. Using spectral unmixing, dyes with even closer spectra can be used with only one excitation and STED laser [41]. Spectral unmixing, however, gets increasingly difficult when the structures of interest co-localize and great care has to be taken in the analysis.

Other dye properties have been used for multi-color STED microscopy: Photoswitchable fluorescent proteins with a similar spectrum yet opposite switching behaviour allow a sequential imaging using the same lasers [42]. Fluorescence lifetime can be used for imaging two dyes with a very similar spectrum simultaneously [38].

However, separating dyes by their spectrum has proven to be the most robust and flexible method. Several dyes covering the whole visible wavelength range have been shown to be 


\section{INTRODUCTION}

applicable for STED microscopy. Organic dyes with emission spectrum in the red and near infrared region are relatively photostable. At the same time, the absorption cross section for the STED wavelengths above $700 \mathrm{~nm}$ is very low in most biological samples, allowing high powers to be applied.

The goal was therefore to apply the concepts of pulse-interleaved excitation/detection to minimize the spectral crosstalk, and the use of a single laser for STED on spectrally distinct red and far-red dyes $[38,43]$ to build a two-color STED microscope.

\subsubsection{Scanning a Field of View Below the Diffraction Limit}

Ultimately, the accuracy of every experiment is limited by its signal-to-noise ratio. In fluorescence microscopy, this limit is defined by the number of photons available from each fluorophore. While the equations for the resolution (1.1),(1.4) provide a good estimate, the actual resolution is lowered by signal noise. In imaging, the shot noise of the arriving photons limits the signal-to-noise ratio to

$$
\mathrm{SNR}=\sqrt{N}
$$

where $N$ is the number of measured photons. The image of two close sample points is the addition of both PSFs (Figure 1.1b). The Rayleigh criterion defines points as resolved when the intensity drops by $\approx 25 \%$ in-between the peaks. To reliably see the intensity drop in the signal, the SNR has to be considerably higher than 4, requiring a peak intensity of well above 16 photons in the image.

Which leads directly to another important point in imaging: The sampling size. One data point in the image is the integrated signal of a certain area in the sample. When the sampling size is below the resolution, the signal of a fluorophore spreads over many pixels, lowering the SNR. Choosing a sampling size above the resolution will not resolve the signal drop inbetween two adjacent molecules. The pixel size should therefore be at least $2.4 \times$ smaller than the resolution [44].

The above mentioned points pose a fundamental challenge for point scanning super-resolution methods: The resolution is usually increased with the intensity of the localizing laser pattern. High intensities increase photobleaching, limiting the available fluorescence signal. Additionally, the sampling size has to be reduced along with the resolution, resulting in longer irradiation times of the same area. A two-fold improvement in the resolution will cause a four-fold longer imaging and irradiation time. As the PSF size is narrowed, fewer fluorophores contribute to the measured signal, and eventually the sampling time has to be increased. Finding the optimal combination of resolution, pixel size, sampling time and laser powers for a specific sample is the everyday challenge a microscopist has to face.

As the available signal is always limited by photobleaching, strategies have been developed to reduce it. Photobleaching is a chemical reaction of the dye with its surroundings and can be reduced by using appropriate sample buffers [45]. However, this is not always applicable, for example when measuring in living cells. Technical strategies primarily developed for STED microscopy reduce the bleaching by allowing dark state relaxation of the molecule (D-Rex, [46]) and reducing the state transition cycles (RESCue, [47]).

D-Rex reduces the bleaching caused by higher order excitation from long-lived triplet or dark states, the rate of the excitation cycles is slowed down enough to ensure that the fluorophores can relax to their ground state. The method used to be connected to very low 
laser pulse repetition rates and accordingly long acquisition times, yet has been improved recently by implementing extremely fast beam scanning $[48,49,50]$.

RESCue reduces the total switching cycles of a dye by conditionally switching off the laser irradiation during the signal acquisition of one pixel. A designated electronics module registers the number of photons acquired while imaging and blocks the laser irradiation either (a) when a certain threshold is not reached within a specified time interval, i.e. there is no dye in the sampled volume; or (b) when a certain threshold is exceeded, i.e. enough signal has been acquired to extrapolate the brightness. Because the sampling volume is only a fraction of the irradiated volume, switching the lasers off for individual pixels avoids bleaching of the surrounding area.

Limiting the field of view to an area smaller than the irradiating laser foci is another way to reduce the switching cycles of the fluorophores. The idea is illustrated in Figure 1.6: While the excitation and STED laser foci are several hundred nanometer in diameter, many biological structures are in the order of $100 \mathrm{~nm}$ in size. The large scale distribution of the labeled protein can often be imaged satisfactory with confocal resolution or low STED power, then spots of interest can be selected and the organization on the nanoscale is investigated on a small area not exceeding $\approx 200 \mathrm{~nm}$. Selectively scanning an area smaller than the laser foci avoids switching off the fluorophores in that area when scanning over adjacent sample points in a normal scan. For sufficiently small areas, irradiation with the high-intensity doughnut maxima is also avoided.

Implementing this idea into a STED microscope and characterizing its properties will be the main part of this thesis.

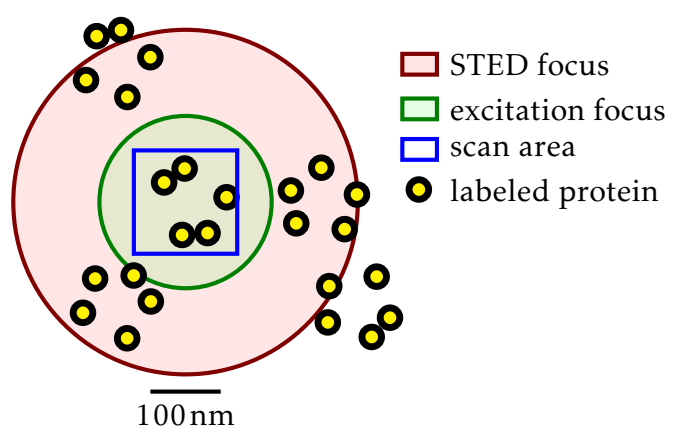

(a)

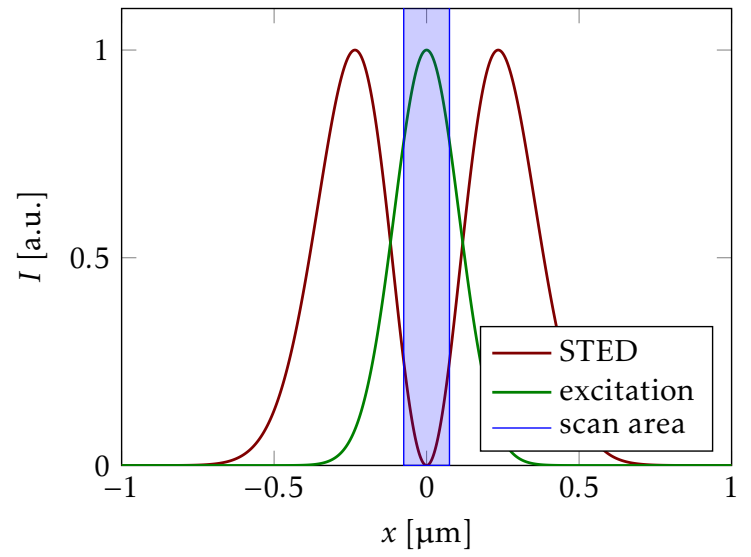

(b)

Figure 1.6. Scanning only a field of view smaller than the laser foci reduces the irradiation of the imaged dyes. (a): Schematic of the scan field in the sample. (b): Scan field in relation to the laser intensities. 



\section{Methods}

\section{Fluorescent Dyes}

The following fluorescent dyes were used in the course of this thesis:

$\begin{array}{lll}\text { Dye } & \begin{array}{l}\text { Absorption / } \\ \text { emission maximum [nm] }\end{array} & \text { Manufacturer } \\ & & \\ \text { Atto 647N } & 647 / 670 & \text { ATTO-TEC, Siegen, Germany } \\ \text { Atto 590 } & 594 / 624 & \\ \text { Atto 594 } & 594 / 627 & \\ \text { Star 635P } & 634 / 654 & \text { Abberior, Göttingen, Germany } \\ \text { Star Red } & 637 / 660 & \\ \text { (KK114) } & & \\ \text { Star 580 } & 584 / 607 & \end{array}$

\section{Single Molecule Sample Preparation}

Prior to the sample preparation, the cover glasses were sonicated in 2\% Hellmanex III (Hellma Analytics, Müllheim, Germany) solution for 30 minutes to remove possibly fluorescent contaminations.

The samples for the single molecule measurements were prepared following a modified version of the protocol described in $[51,52,53]$ : A sample chamber was prepared as depicted in Figure 2.1. The chamber was filled with $30 \mu \mathrm{L}$ biotin-conjugated bovine serum albumin (BSA) solution $(0.5 \mathrm{mg} / \mathrm{mL}$, Sigma Aldrich, St. Louis, MI, USA) and incubated for $5 \mathrm{~min}$ -

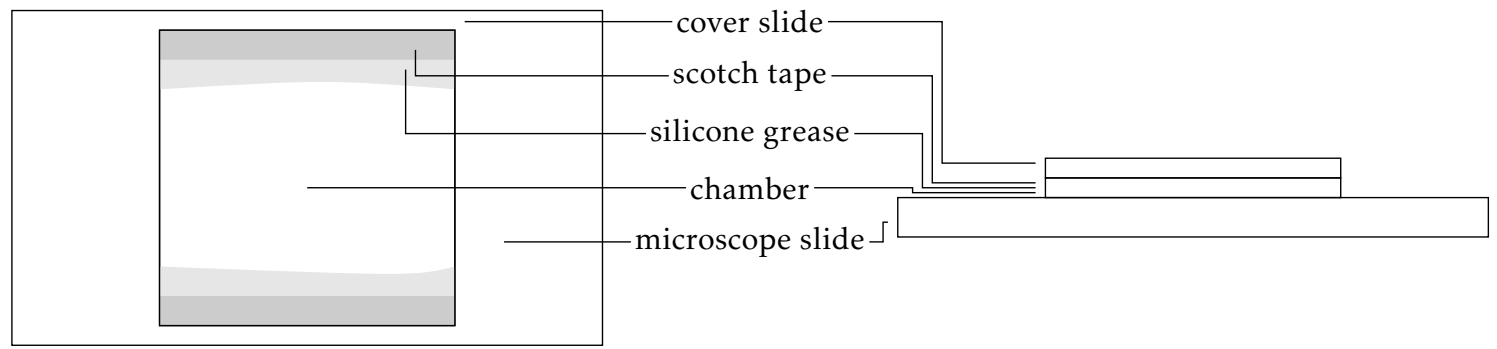

Figure 2.1. Preparation of the single molecule sample. Double-faced scotch tape was used to hold the coverslip and provide a spacing between the microscope slide. Before mounting the coverslip, silicone grease was spread next to the scotch tape to avoid diffusion of glue and other chemicals into the sample chamber, as well as to prevent drying of the sample. Solutions were added to the chamber by pipetting them to one of the open sides and removing the fluids on the other side with a paper wipe. After the sample preparation the sides were sealed with silicone grease. 


\section{Methods}

utes, then washed with $200 \mu \mathrm{L}$ PBS. The PBS was replaced with $30 \mu \mathrm{L}$ streptavidin solution $(0.5 \mathrm{mg} / \mathrm{mL}$, Roche Diagnostics, Mannheim, Germany), followed by another washing step. The biotin-labeled BSA attaches to the cover glass surface. Streptavidin has four binding sites with a high affinity to biotin. It binds to the immobilized biotin molecules on the glass, thus providing an anchor for the biotin tagged dsDNA added in the next step. The sample was then checked for undesired fluorescent molecules on the cover glass surface. The chamber was flushed with $30 \mu \mathrm{L}$ labeled dsDNA solution (10 pmol, IBA, Göttingen, Germany). The double-stranded DNA consisted of 31 base pairs where the $5^{\prime}$ ends of the strands were tagged with one Atto647N molecule and biotin respectively. The biotin couples to the streptavidin, immobilizing the DNA construct. If the density of immobilized dyes was too small, more dye-DNA solution could be added, followed by a washing step. In principle, 3 DNA strands can couple to one immobilized streptavidin. Yet the low concentration of the dye solution makes it unlikely to have more than one DNA molecule per streptavidin. The sample was sealed with a silicone based grease (high vacuum grease, Dow Corning, Midland, MI, USA). It was chosen for its inert properties and high hydrophobicity. Other sealants tested (two component epoxies, two component silicone rubber) did not result in the desired sealing performance or disturbed the sample behavior.

\section{Nuclear Pore Samples}

The single-color and two-color gp210 / Pan-FG samples were prepared by Christian Wurm and Ellen Rothermel. The primary antibodies were provided by Volker Cordes from the Research Group Nuclear Architecture, Max Planck Institute for Biophysical Chemistry (Göttingen, Germany). Xenopus laevis X177 and A6 cells were fixed with formaldehyde and treated with Triton $\mathrm{X}$ and bovine serum albumin before incubation with the primary antibodies. Commercial secondary antibodies (Dianova, Hamburg, Germany) labeled with above mentioned dyes are used to detect the primary antibodies. The samples were mounted in Mowiol. The detailed sample preparation can be found in [54].

The nanobody samples were prepared by Tino Pleiner from the Department of Cellular Logistics, Max Planck Institute for Biophysical Chemistry. The production and labeling protocol of the nanobodies is currently in review for publication [55]. Xenpus laevis XL117 were permeabilized with digitonin, followed by incubation with 1-10 nmol solution of labeled nanobodies. The cells were fixed with formaldehyde and mounted in Mowiol. The detailed preparation protocol will be available in the publication.

\section{Fluorescent Beads}

The samples with fluorescent beads were prepared by diluting the stock solution of $20 \mathrm{~nm}$ crimson FluoSpheres (Invitrogen / Thermo Fisher Scientific, Waltham, MA, USA) by 1:10 000, followed by sonication. A coverslip was coated with poly-L-lysine, washed and incubated with the bead solution for a few minutes. The coverslip was mounted with Mowiol on the object slide.

\section{Area Sizes}

One of the most important parameters of the experiments in this thesis is the scan area size. If the area size is given in units of micrometer or nanometer, the scan area is a square with the indicated side length. 


\section{Two-Color STED Microscopy}

Imaging separate structures in a cell simultaneously is a key tool to understand their interaction. STED microscopy offers the technique to do so with a resolution below $30 \mathrm{~nm}$, yet the laser wavelengths and dyes used have to be matched. Channel crosstalk and co-localization accuracy are major limitations to the information the images can provide.

In this chapter, a two-color STED microscope is presented that was designed to provide superior resolution in two color channels with minimal crosstalk and maximal co-localization accuracy.

\subsection{Setup Design}

The layout of the setup is sketched in Figure 3.1 and the most important components are listed in Table 3.1. The configuration described here is the most recent, it was repeatedly modified in details (excitation laser model, APD model, filter wavelengths, ...).

The STED laser (ELP-5-775-DG, IPG Photonics Corporation, Oxford, MA, USA) is a frequencydoubled fiber laser providing reliable performance. It emits pulses of $1.2 \mathrm{~ns}$ length at a wavelength of $775 \mathrm{~nm}$ and $20 \mathrm{MHz}$ repetition rate. To achieve the typical doughnut shaped focus, the STED laser passes a $2 \pi$ vortex phase plate (RPC Photonics, Rochester, NY, USA) and is then aligned with the other beam paths on the main axis. Before entering the objective, its polarization is circularized with a $\lambda / 4$ waveplate. The quality of the circular polarization is essential for the quality of the central intensity zero. The $\lambda / 4$ waveplate is therefore mounted on an additional horizontal rotation mount to correct chromatic dependence of the retardation by angle tuning.

For efficient super-resolution, the excitation pulses need to be much shorter than the STED pulse length and their arrival time in the sample must be synchronized with the STED pulses. They are generated by laser diodes at a wavelength of $640 \mathrm{~nm}$ (LDH-D-C 640, PicoQuant, Berlin) and 594nm (custom built, [56]) with pulse lengths $\leq 100 \mathrm{ps}$. The STED pulse timing has a jitter of $>1 \mathrm{~ns}$, thus it was necessary to trigger the excitation pulses directly to the according STED pulse. A photodiode with constant fraction discriminator measured the arrival time of the STED pulses and provided the trigger signal for the excitation. To compensate the delay between trigger pulse arrival and emission of the corresponding excitation pulse, the STED beam was coupled into a $30 \mathrm{~m}$ long, polarization-maintaining single-mode fiber. The coarse adjustment of the pulse arrival times at the objective was controlled via cable and beam path lengths, the fine tuning via a (home built) electronic delay unit. The unit featured two independent delay channels for adjusting the pulse timing, as well as a frequency divider. The incoming $20 \mathrm{MHz}$ signal was converted to $10 \mathrm{MHz}$ where the rising and falling signal edge was triggered by successive input pulses. The two excitation lasers were triggered with the rising/falling edge of the signal, as was the time gate (see below), resulting in a pulse-interleaved excitation and detection scheme. This scheme allowed quasisimultaneous two-color imaging with low channel crosstalk [57] (see also Section 3.2.1). 


\section{Two-Color STED Microscopy}

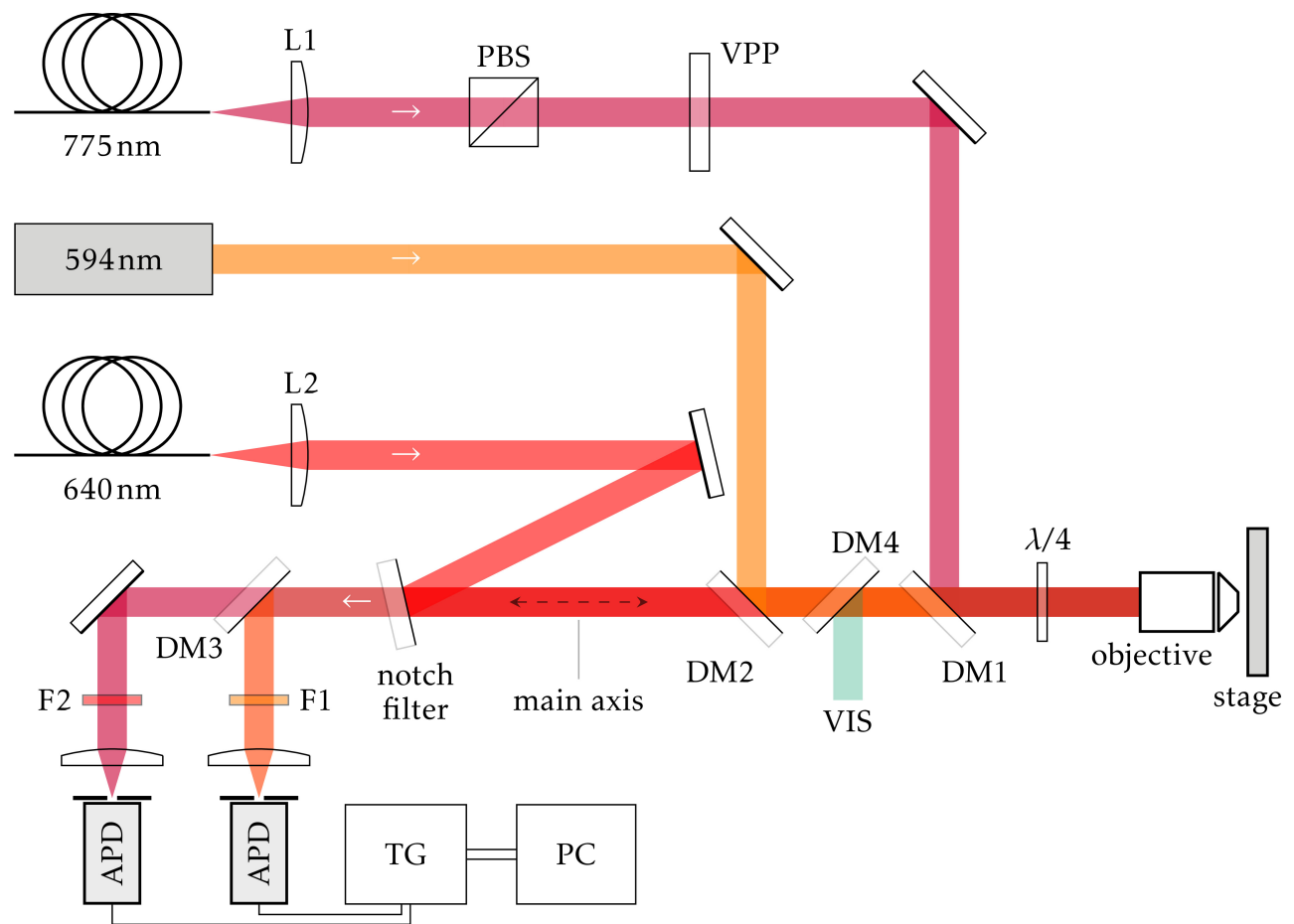

Figure 3.1. Outline of the two-color STED microscope. The setup is based on a pulsed STED laser with a wavelength of $775 \mathrm{~nm}$, two excitation lasers and detection channels. A third (confocal) channel (VIS) is configured for dyes in the visible wavelength range.

All beam paths are overlaid on the main axis with suitable dichroic mirrors (DM, notch filter for the $640 \mathrm{~nm}$ excitation laser) and aligned for confocal excitation and detection. A polarizing beam splitter (PBS) ensures a clean polarization of the STED beam. It then passes a $2 \pi$ vortex phase plate (VPP) and a $\lambda / 4$ waveplate which in combination leads to the typical 'doughnut' shaped focus with a central intensity zero. The fluorescence signal is collected by the objective, selected for wavelength by DM3 and filters F1, F2, and detected by avalanche photodiodes (APD). The APD signal is time gated (TG), i.e. signal pulses outside a certain time window are discarded, and sent to the computer (PC) for pulse counting. An image is acquired by scanning the sample with a piezo stage and mapping the measured photon counts to the sample position.

\begin{tabular}{|lr|}
\hline L1 & achromat, $f=30 \mathrm{~mm}$ \\
L2 & achromat, $f=60 \mathrm{~mm}$ \\
DM1 & z760SPRDC \\
DM2 & z585RDC \\
DM3 & z635RDC \\
DM4 & z545DCXR \\
Notch filter & stop line 658 nm \\
F1 & HQ 620/40 \\
F2 & HQ 690/60 \\
$\lambda / 4$ & $500-900 \mathrm{~nm}$ achromat \\
Objective & PL APO HCX 100x 1.4-0.7 Oil \\
\hline
\end{tabular}

Table 3.1. List of central optical components. DM1-4, F1 and F2 manufactured by Chroma Technology, Bellows Falls/VT; notch filter by Semrock, Rochester, NY; objective by Leica Microsystems, Wetzlar; $\lambda / 4$ by B. Halle Nachfl., Berlin 
The $640 \mathrm{~nm}$ excitation laser was coupled into a polarization-maintaining single-mode fiber before entering the setup to clean the beam profile. It was sufficient to expand the beam of the $594 \mathrm{~nm}$ diode directly in the setup. A third color channel providing confocal resolution for dyes with an emission between $500-550 \mathrm{~nm}$ was added: A CW diode laser provided the excitation light at $470 \mathrm{~nm}$, the fluorescence was detected with an avalanche photo diode (APD).

The setup is optimized for red and far-red dyes that have their emission maxima between $600-640 \mathrm{~nm}$ and $650-700 \mathrm{~nm}$ respectively. The color channels are separated the following way (see also Figure 3.2): The $594 \mathrm{~nm}$ laser inevitably excites both dyes and both will fluoresce above $650 \mathrm{~nm}$, yet only the red dye will be fluorescent in the $600-640 \mathrm{~nm}$ detection channel. The far-red dye can be selectively excited with the $640 \mathrm{~nm}$ laser and its fluorescence is detected above $650 \mathrm{~nm}$. This principle has been implemented on a pulse-to-pulse basis, i.e. each excitation laser runs at $10 \mathrm{MHz}$ while their timing is shifted by $50 \mathrm{~ns}$.

The $640 \mathrm{~nm}$ laser has to be coupled into the main beam path with a filter that leaves most of the fluorescence spectrum free in order to achieve a good detection efficiency. Therefore, a notch filter was used in the $640 \mathrm{~nm}$ beam path. Notch filters offer a high transmission outside a narrow (here: $<30 \mathrm{~nm}$ ) highly reflective band. The high reflectivity additionally allows to extend the red detection band up to $640 \mathrm{~nm}$. The notch filter and DM2 (595 path) were angle-tuned to transmit as much of the fluorescence signal in this window as possible.

The fluorescence signal is collected by the objective and detected by two avalanche photo diodes (fiber coupled SPCM-AQRH13, Excelitas,Waltham, MA, USA; free beam PR-50-CTB, Micro Photon Devices, Bolzano, Italy). The fiber opening acts as a confocal pinhole, a separate pinhole was implemented for the free beam APD. The size of the pinhole is $\approx 1$ Airy disk. The signal of the APDs is processed by a time gate (home built) for pulse-interleaved detection and removing undesired signal [58]: The time gate discards signal outside a $10 \mathrm{~ns}$

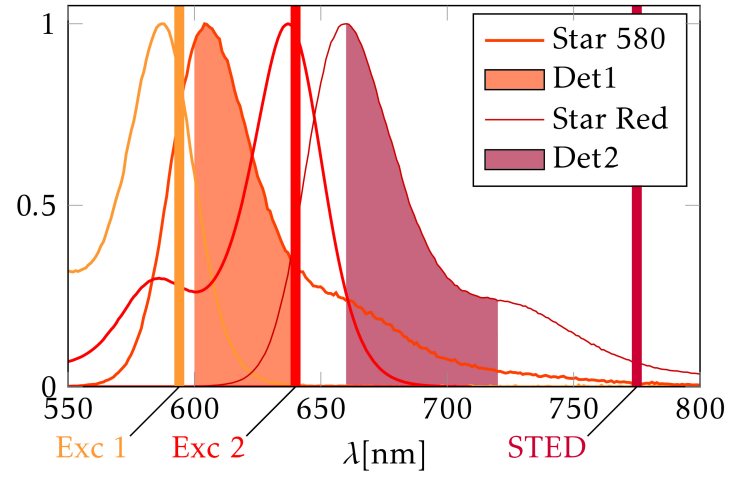

(a)

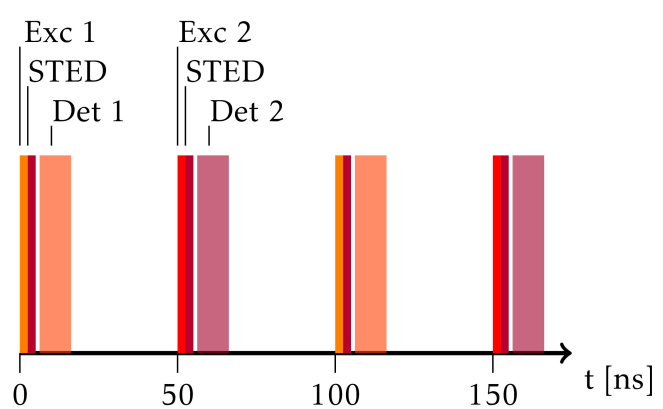

(b)

Figure 3.2. Spectral (a) and temporal (b) excitation and detection scheme. The fluorescence signal is separated by wavelength on two detectors (Det 1: 600-640 nm; Det 2: 660-720nm). The pulses of each excitation laser (wavelengths Exc 1: $594 \mathrm{~nm}$; Exc 2: $640 \mathrm{~nm}$ ) are triggered by every second STED pulse and the detector signal is additionally time gated: Signal outside a $10 \mathrm{~ns}$ window after the corresponding excitation pulse is discarded. This allows quasi simultaneous recording of both color channels while minimizing spectral crosstalk. 


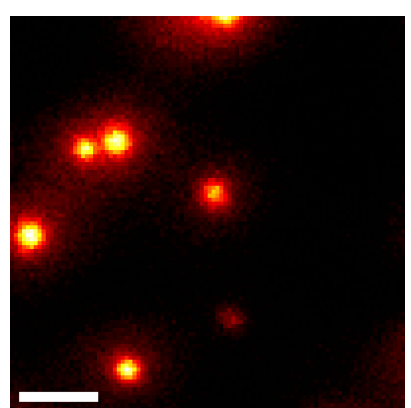

(a) without time gating

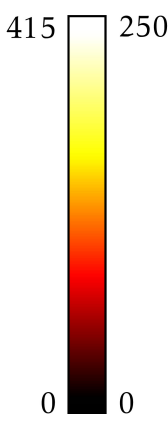

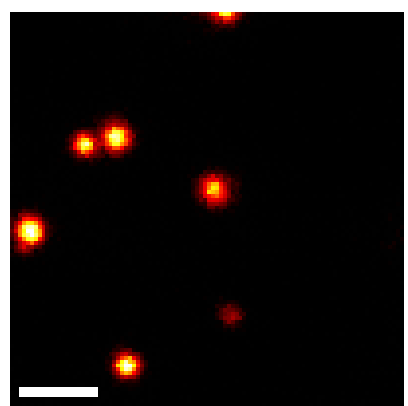

(b) with time gating

Figure 3.3. Comparison between regular (a) and time-gated detection (b). Because of the long STED pulse duration $(\approx 1 \mathrm{~ns})$, spontaneous emission already occurs during its action, leading to a fuzzy background. Discarding this part of the signal by time gating reduces the total signal, yet increases the contrast. Images are of fluorescent beads, scale bar: $200 \mathrm{~nm}$

window after a trigger pulse. The trigger pulse is delayed by $\approx 1$ ns to the arrival time of the excitation pulse. During this time the STED pulse still acts on the sample and spontaneous emission from yet unquenched dyes blurs the resulting image (see Figure 3.3). Some of the desired fluorescence signal is lost, yet the signal-to-noise ratio is usually better with timegated detection, as additionally the dark count rate is reduced 10 -fold (for $10 \mathrm{MHz}$ excitation rep. rate). The fine tuning of the gate delay can be adjusted to the needs of the sample for optimal results.

The image is created by scanning the sample with a 3-axis piezo stage (Nano-PDQ, Mad City Labs, Madison, WI). A data acquisition card (NI-PCIe 6353, National Instruments, Austin, TX) synchronizes the sample movement and photon counting. The software Imspector [59] configures the measurement process and provides a graphical user interface.

\subsection{Results}

We characterized and demonstrated the capabilities of the two-color STED microscope in imaging and fluorescence correlation spectroscopy. The results have been published in [54] and are presented below.

\subsubsection{Channel Crosstalk}

The stimulated emission cross section of a dye is wavelength dependent and roughly proportional to the emission spectrum of the dye [60]. Therefore, in order to achieve superresolution by stimulated emission depletion, all used dyes have to be fluorescent at the STED wavelength. This introduces spectral crosstalk of the dyes in the excitation and detection channels. If the crosstalk is too strong, the channel can be unmixed in a post-processing step. However, the unmixing algorithms may introduce artifacts and do not work well when the signal is noisy or structures mostly co-localize.

The crosstalk of the microscope was measured on immunolabeled nuclear pores (pan specific primary antibody against NUP153, NUP214 and NUP62; labeled secondary antibody) mounted in Mowiol for the dyes Star Red (also referred to as KK114), Star635P, Atto594, 


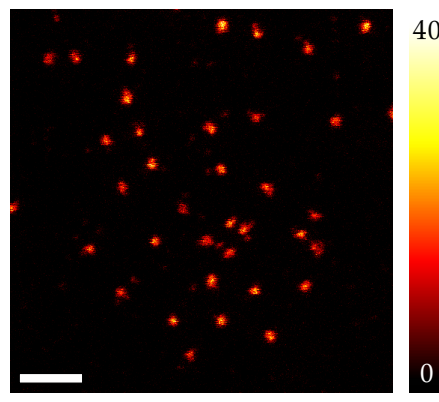

(a) $690 / 60$

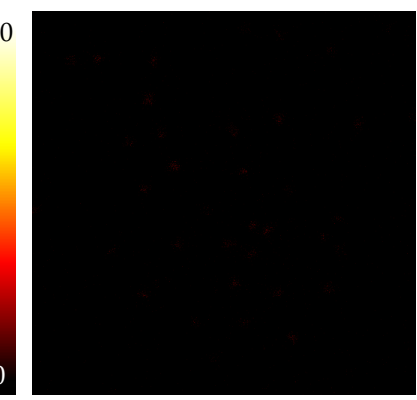

(b) $620 / 40$

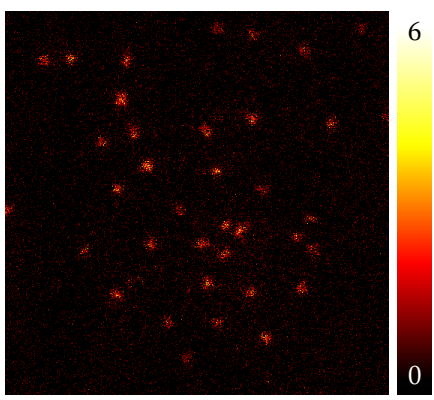

(c) $620 / 40$

Figure 3.4. Crosstalk of the dye Star Red. Approximately 7\% of the signal (a) bleeds into the $620 / 40$ detection channel (b, $\mathrm{c}$ with different color scale). This poses no problem when the labeling with the orange dye is of comparable brightness and no unmixing is required. Scale bar: $500 \mathrm{~nm}$

\begin{tabular}{|lcc|}
\hline dye & main detection channel & crosstalk (\%) \\
Star Red & $690 / 60$ & $7.2 \pm 0.1$ \\
Star635P & $690 / 60$ & $4.7 \pm 0.1$ \\
Star580 & $620 / 40$ & $0.84 \pm 0.06$ \\
Atto590 & $620 / 40$ & $3.6 \pm 0.2$ \\
Atto594 & $620 / 40$ & $8.7 \pm 0.2$ \\
\hline
\end{tabular}

Table 3.2. Channel crosstalk of the most commonly used dyes on the two-color setup.

Atto590 and Star580. The laser power was set to $4 \mu \mathrm{W}$ for both excitation wavelengths and $140 \mathrm{~mW}$ for the STED laser.

A binary mask was created by thresholding the smoothed image of the correct color channel. The raw data of both color channels was subsequently masked, the signal summed and background subtracted. The crosstalk is the quotient of the integrated signals. Errors were calculated by assuming a Poisson distribution of the fluorescence signal and dark counts. The results are summarized in Table 3.2. For the tested dyes the crosstalk stayed below $10 \%$. This amount is hardly noticeable in the resulting image if the sample is labeled with similar brightness in both color channels (Figure 3.4). Atto594 and Star Red exhibit the highest crosstalk but were used in many experiments as they provided the (subjectively) best image quality. One should be aware that the spectral properties of fluorescent molecules also depend on their environment. Changes in labeling density, labeled structure, mounting medium etc. may affect the crosstalk.

\subsubsection{Co-localization Accuracy}

The main application of two-color imaging is to investigate how two structures localize with respect to each other. However, the two image channels might not overlap perfectly. Common causes for the mismatch are:

- Misaligned excitation and detection channels

- Chromatic aberrations

- Sample drift (in case of sequential imaging) 


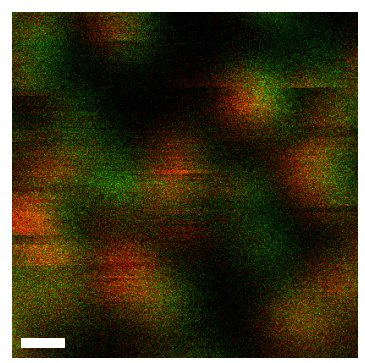

confocal

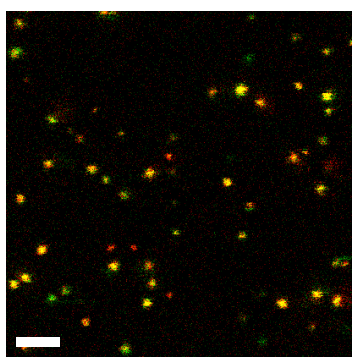

STED

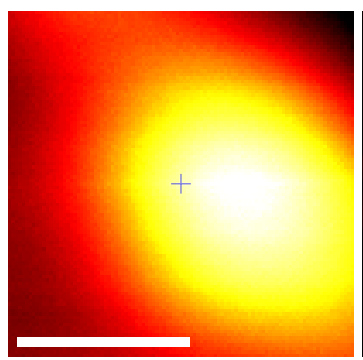

confocal

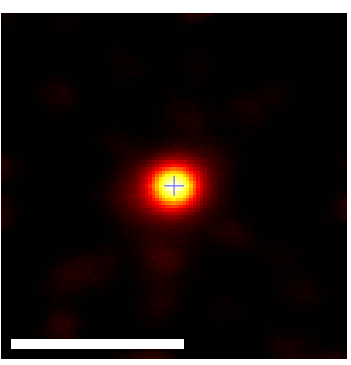

STED

(a) image

Figure 3.5. Image of $20 \mathrm{~nm}$ sized crimson beads and cross-correlation of the channels. A physical misalignment will lead to a channel shift in the resulting confocal image. The use of a single STED doughnut for both channels drastically improves the co-alignment in the STED image. A gaussian fit of the cross correlation yields a channel shift below $1.5 \mathrm{~nm}$ in the STED image (data not shown). Scale bar: $250 \mathrm{~nm}$

These factors have to be considered and corrected for, usually by imaging a well defined test sample and post processing the data. But with increasing resolution, even small thermal drifts during image acquisition may be noticeable. On the presented microscope, these effects are minimized by the use of one single STED doughnut for both color channels and the pulse-interleaved excitation/detection scheme. As the fluorescence in each channel can only originate from a small area in the doughnut center, moderate misalignment has little effect on the co-localization accuracy. The signal of both channels is recorded sequentially within $100 \mathrm{~ns}$ - regular sample drift occurs on the time scale of seconds. The channel shift in the resulting STED image is an order of magnitude smaller than the resolution, even for substantial misalignment of the excitation lasers (Figure 3.5). A post acquisition correction is therefore not necessary.

\subsubsection{Resolution}

Resolution is the main criterion every microscope has to compete in. It limits the level of detail that is observable and is defined as the minimal distance between two point-like objects at which the objects can still be identified as separate in the final image. A direct measurement of the resolution according to this definition is challenging: One would require a very well defined test sample with points at various distances. Apart from the size of the microscopes PSF, the signal-to-noise ratio is a limiting factor for resolution, yet varies from sample to sample. In practice one tries to estimate the size of the PSF by measuring small objects like fluorescent beads or unbound antibodies.

Here, we measured the size (FWHM) of dye clusters directly in a biological sample (see A.1) as well as the diffusion time through the focus in FCS measurements (see below). The achievable resolution was found to be $\approx 20 \mathrm{~nm}$ for the red and $\approx 30 \mathrm{~nm}$ for the green color channel.

\subsubsection{Flourescence Correlation Spectroscopy (FCS)}

FCS is a powerful tool to investigate dynamics in biology and chemistry ( [9], [61]). It makes use of the high temporal resolution at which a fluorescence signal can be acquired. By (auto-) 
correlating the measured signal, one can derive typical time constants of the sample. Here, the diffusion constant of lipids tagged with Star Red was measured. The auto-correlation $G(\tau)$ of the fluorescence signal $S(t)$ is defined as [62]:

$$
G(\tau)=\frac{\langle S(t) S(t-\tau)\rangle-\langle S(t)\rangle^{2}}{\langle S(t)\rangle^{2}}
$$

By analyzing $G$ for different environmental conditions, one can draw conclusions about the structure the dye is diffusing through, for example a cell membrane: As the diffusion is influenced by molecular interactions between membrane components, tagging a certain protein or lipid with a dye gives insights into the complex dynamics in cells.

STED microscopy offers a resolution of few $10 \mathrm{~nm}$ and the unique ability to easily tune the size of the detection volume by changing the STED laser power. The diffusion behavior of a probe can be studied on different length scales, which is important to understand the investigated structure [63].

If the sample features a well-known behavior, one can characterize the measuring system by comparing the predicted to the experimental data. We used lipid diffusion in a glasssupported lipid bilayer as a model system for free diffusion. The autocorrelated signal was fitted with a two-dimensional anomalous diffusion model [64] (Figure 3.6a):

$$
G(\tau)=\frac{1}{N}(1 * T(1-T)) \exp \left(-\frac{\tau}{\tau_{T}}\right) \frac{1}{\left(1+\frac{\tau}{\tau_{D}}\right)^{\alpha}}
$$

where " $N$ denotes the average number of fluorescent molecules in the detection volume, $T$ the fraction of molecules that are on average in the dark triplet state, $\tau_{T}$ the triplet dwell time, $\tau_{D}$ the average transit time of the molecules diffusing through the observation area, and $\alpha$ the anomalous diffusion exponent" [54].

The diffusion time $\tau_{D}$ through the detection area $A_{\text {det }}$ scales linearly with the size of $A_{\text {det }}$ for free diffusion. Fitting of the data yields $\alpha>0.8$, which can still be considered unhindered diffusion. With the size of the detection volume FWHM $\sim \sqrt{A}$, we can calculate the resolution with:

$$
\operatorname{FWHM}\left(P_{\mathrm{STED}}\right)=\operatorname{FWHM}\left(P_{\mathrm{STED}}=0\right) \sqrt{\frac{\tau_{D}\left(P_{\mathrm{STED}}\right)}{\tau_{D}\left(P_{\mathrm{STED}}=0\right)}}
$$

where the confocal resolution $\operatorname{FWHM}\left(P_{\text {STED }}=0\right)=245 \mathrm{~nm}$ was experimentally determined on fluorescent beads. The results are shown in Figure 3.6b. As equation (1.5) predicts, the resolution scales proportional to $\sim\left(1+P_{\mathrm{STED}} / P_{0}\right)^{-\frac{1}{2}}$ (gray line). We could show that a resolution of $15 \mathrm{~nm}$ is feasible at $480 \mathrm{~mW}$ STED power. More STED power resulted in too little signal as the diffusion time through the detection volume is small, the number of dyes diffusing through is low and the dye molecules may bleach before reaching the detection volume.

To demonstrate the applicability to live cell measurements, we have also investigated the diffusion behaviour of a fluorescent glycerophospholipid analog (DSPE-PEG-KK114, [65]) in the membrane of living PtK2-cells with a resolution down to $20 \mathrm{~nm}$. We verified that the diffusion of the lipid is unhindered on length scales of $20-250 \mathrm{~nm}$ (data not shown, see publication for details). 


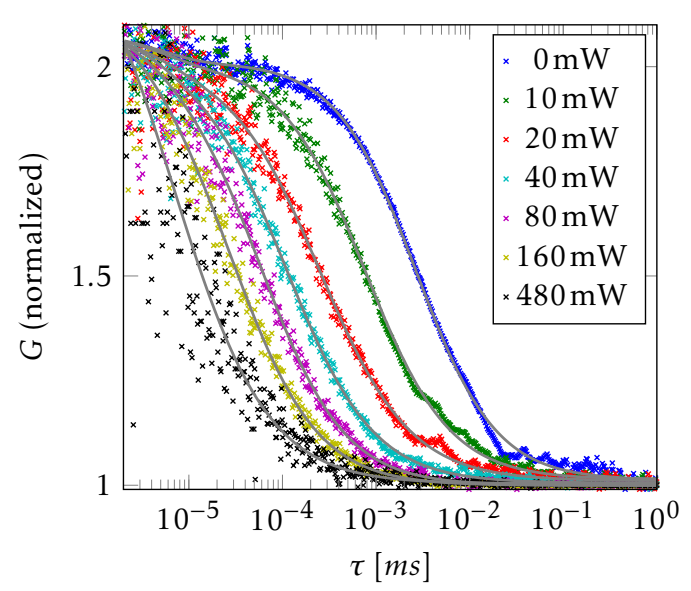

(a)

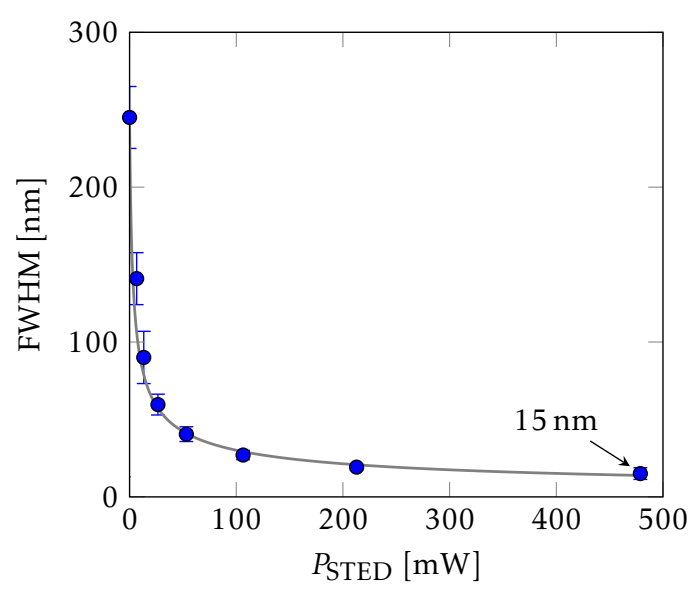

(b)

Figure 3.6. STED-FCS data for a lipid bilayer. The auto-correlated fluorescence signal (a) provides information on the diffusion time through the detection area. The data can be used to derive the resolution for each STED power (b).

The FCS measurements were carried out with Alf Honigmann and Veronika Müller who also prepared the samples and analyzed the data.

\subsubsection{Imaging}

The described properties of the microscope, its flexibility and reliability led to several successful collaborations that are listed in appendix Section A.5. We have successfully imaged various sample types, ranging from thin cell sections embedded in melamine to living brain slices.

One of the most promising applications of optical super-resolution microscopy is the investigation of the nuclear pore complex (NPC). The NPC is one of the biggest protein complexes in a cell with a size of $\approx 160 \mathrm{~nm}$ in diameter. Nuclear pores regulate the transport in and out of the nucleus. They form a diffusion barrier and actively transport larger cargo. Their structure has been studied extensively with electron microscopy yet many aspects of its functionality are not well understood [66]. Electron microscopy requires a harsh sample preparation and cannot study the sample in its natural environment, which is especially important for highly dynamic structures like the NPC.

With the advent of super-resolution microscopy it became possible to investigate the detailed substructure of the NPC in a much less invasive manner [67, 68]. We have demonstrated the capabilities of STED microscopy for imaging nuclear pores on fixed Xenopus A6 and X177 cells. The pores were labeled by indirect immunostaining using a monoclonal antibody specific to the nucleoporin gp210 and a pan-specific antibody targeting most FG repeat proteins ${ }^{1}$, especially Nup214, Ganp, CG1, Nup98 and Nup153. The experiments on nuclear pores were carried out in cooperation with Volker Cordes and Christian Wurm.

gp210 is one of the two integral membrane proteins in vertebrate NPCs. Evidence suggests that its functions are evolutionary conserved [69], implying the critical role of gp210. It is

\footnotetext{
${ }^{1}$ These proteins have many repetitions of phenylalanine-glycine (FG) pairs which are unfolded
} 


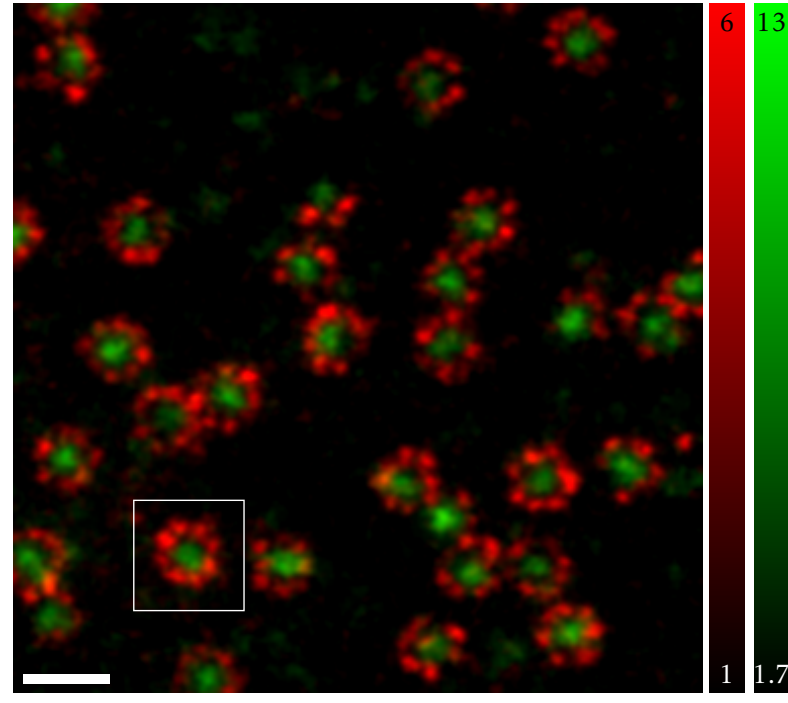

(a)
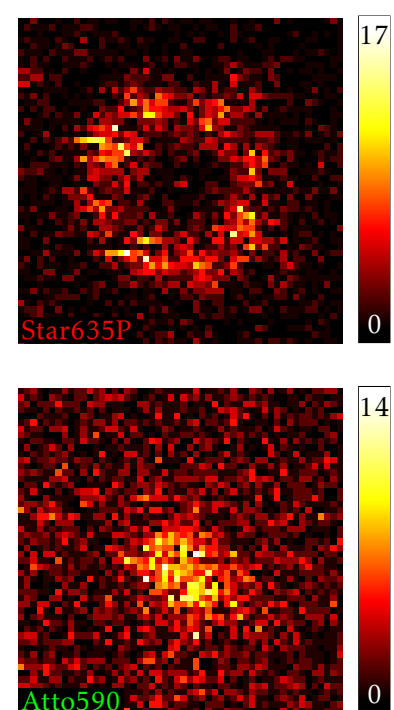

(b)

Figure 3.7. STED image of nuclear pores in Xenopus A6 cells. The nucleoporins gp 210 (red) and several proteins closer to the central channel (green) were labeled with antibodies. The image (a) was smoothed by convolving with a Gaussian of size $\sigma=9 \mathrm{~nm}$. (b) shows the raw data of the highlighted area. The eight subunits of the nuclear pore complex are clearly distinguishable. However, low signal-to-noise and unspecific background complicate a quantitative analysis. Scale bar: $250 \mathrm{~nm}$

located in the periphery of the NPC and adapts its typical 8-fold symmetry, likely providing structural support. The targets of the pan-specific antibody are located closer to the center of the NPC. The FG repeats are natively unfolded nucleoporin segments that form a diffusion barrier in the pore's center and serve as docking sites for transport receptors. Their exact functionality is still unclear [70].

The primary antibodies were targeted with specific secondary antibodies labeled with Star 635P (gp210) and Atto590 (panspecific antibody). The labeling protocol is described in detail in the publication [54]. The images were acquired with a pixel size of $6 \mathrm{~nm}, 30 \mu \mathrm{s}$ pixel dwell time, $\approx 4 \mu \mathrm{W}$ excitation and $180 \mathrm{~mW}$ STED laser power. We could clearly identify the 8 -fold symmetry of the nuclear pores and their arrangement around the pores center (Figure 3.7).

Further experiments have been performed to map various proteins in the NPC. Low labeling quality, probably caused by accessibility problems of the antibodies, as well as (suspected) dislocation of the signal from the protein position due to the antibody size impeded reliable quantitative results. New labeling techniques, combined with the increased fluorescence yield of the newly developed small area scanning microscope, will hopefully advance this project further. 



\section{Scanning Field of Views Below the Diffraction Limit}

\subsection{Design of the Experiment}

This section discusses the experimental realization of the small area STED microscope and measurement procedures. Scanning very small areas poses some challenges to the hardware and software: The scanning system has to be able to scan the desired regions with a high speed and accuracy. The measurement program should provide an interface to easily select regions of interest on an overview scan and sort the acquired data.

The easiest way to implement the idea of measuring only small regions without considerable changes to the hardware is to use an electro-optic modulator to switch off the excitation and STED laser at positions that should not be measured. This was tested on the two-color setup described in the previous chapter. The result is shown in Figure 4.1. As a stage scanner has high inertia, the acceleration curves are several $\mu \mathrm{m}$ long. In practice one scan takes $\approx 1 \mathrm{~min}-$ enough time for the sample to drift. The drift over several frames is a few $10 \mathrm{~nm}$ and hardly noticeable in the confocal scan, yet when the STED area is only $\approx 100 \mathrm{~nm}$, precious field of view is lost. Aligning each scan with the confocal signal is difficult, as the feature sizes in the confocal scan are much larger than the required accuracy. Furthermore the excitation should be switched off in proximity to the STED areas to avoid bleaching. One could increase the dwell time to make use of the bleaching reduction in the first scan, however this would cause strongly inhomogeneous bleaching within the small scan field. Modifying the existing setup was therefore not an option.

\subsubsection{Beam Scanning}

Scaling down the typical scan parameters of a regular STED microscope $(10 \mathrm{~nm}$ pixel size, $100 \mu$ s pixel dwell time) to a total scan area of $100 \mathrm{~nm} \times 100 \mathrm{~nm}$ poses some challenges to the scanning system: The line frequency will be in the order of $1 \mathrm{kHz}$ while the field of view should be scanned as linearly as possible. The accuracy and repeatability of positioning must

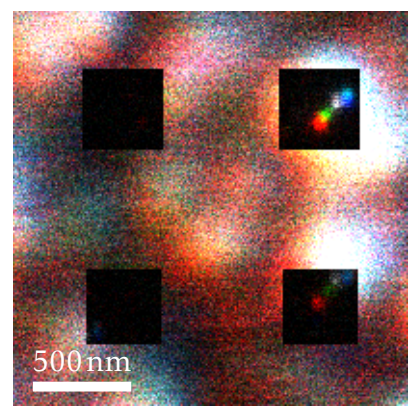

Figure 4.1. Image overlay of an early test of small STED scanning fields on fluorescent beads: The STED laser is switched on at defined positions only ('black' squares), while a regular sized region is scanned with the piezo stage. The scan size was $10 \times 10 \mu \mathrm{m}^{2}$ with $\approx 2 \mathrm{~min}$ between repetitions (colored in red, green, blue, gray, cyan). The drift during the scans would require continuous and nm-precise drift correction, as a small area is ideally scanned $\gg 10$ times. 


\section{Scanning Field of Views Below the Diffraction Limit}

be in the order of $1 \mathrm{~nm}$. Especially challenging is the accurate determination of the desired scan position: All scanners have a certain time lag between the set and actual position. This lag may vary with the scan speed and scan position. Hitting a target with an accuracy below $100 \mathrm{~nm}$, however, is crucial for the usability of the setup.

The scanning system should therefore satisfy the following requirements:

- Minimal aberrations

- Bandwidth of $\gg 1 \mathrm{kHz}$

- Achromatic deflection in a wavelength range $\Delta \lambda>150 \mathrm{~nm}$

- Fast acceleration

- Several micrometer scan range

- Flexible scan speed

- Low / easy to correct lag between set and actual position

- Repeatable and stable positioning $\ll 10 \mathrm{~nm}$

A comprehensive discussion on beam scanning techniques can be found in [71]. Galvanometric and piezo scanning systems have too much momentum to achieve the necessary acceleration without overshooting and oscillations in the linear scan region. Resonant galvanometric scanners provide a good compromise as they are fast and the positioning is reliable. The laser would have to be blanked in the turning points with an electro-optic or acoustooptic modulator to avoid bleaching. However, they lack the flexibility to easily change the scan size and -pattern. Acousto-optic deflectors provide the necessary speed and accuracy yet the deflection is wavelength dependent and the efficiency is low. Less commonly used scan systems have similar disadvantages.

The scanning system that addresses all these points is electro-optic beam scanning, compromising only on the scan range. Electro-optic scanners have no mechanical parts and can steer the beam virtually instantaneously, allowing to easily optimize related parameters such as scan size, scan pattern, scan speed etc. for the specific application. The experimental realization does not require complex synchronization routines, such as laser flyback blanking or lag compensation. As with all beam scanners, the image is acquired by deflecting the beam by an angle $\alpha$ in the objective's back focal plane (Figure 4.2a). For a focal length of $f_{O}$ and small angles, the focus will be moved by:

$$
d=f_{O} \tan \alpha \approx f_{O} \alpha
$$

\subsubsection{Electro-Optic Deflectors}

\section{Theory}

Electro-optic effects describe the changes in optical properties of a medium when an electric field $\vec{E}$ is applied. They are usually dependent on the field orientation to the crystal axes, as well as the propagation and polarization direction of the transmitted light. The linear 


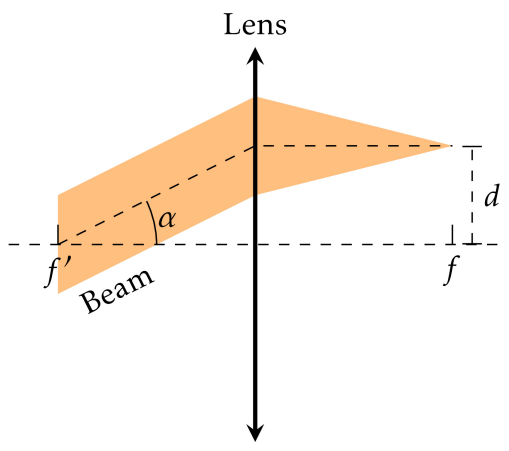

(a)

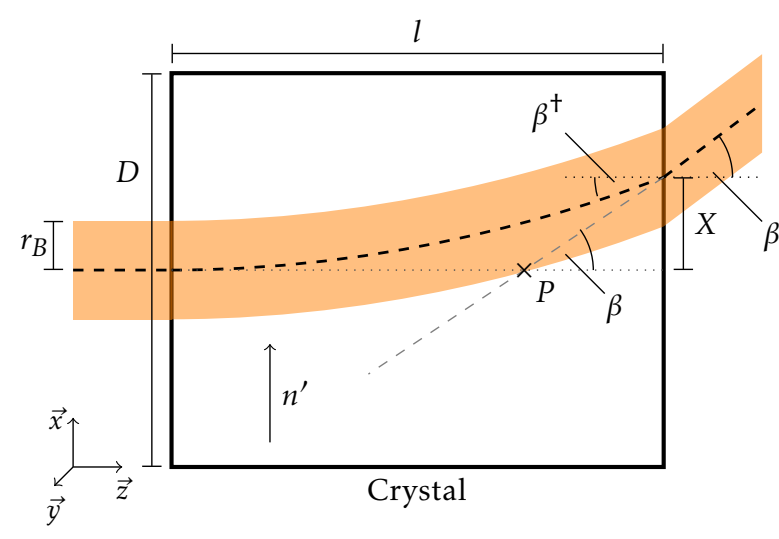

(b)

Figure 4.2. Principle of beam scanning (a) and electro-optic deflection (b). The image in a beam scanning system is acquired by varying the angle of incidence $\alpha$ of the beam, thus displacing its focus by $d$ in the sample plane $f$. The beam should stay centered on the optical axis close to the back focal plane $f^{\prime}$ when tilted. In the presented microscope the beam is scanned by an electro-optic deflector: Applying a linear electric field gradient to an electrooptic crystal will cause a linear gradient $n^{\prime}$ in its refractive index. The beam gets deflected by an angle $\beta^{\dagger}$. At the crystal surface refraction causes an additional deflection to a total angle $\beta$. For an observer outside the crystal it would seem as if the beam is deflected at the pivot point $P$. The pivot point can be projected on the back focal plane of the objective with a telescope, thus keeping the beam centered when scanning.

change in the refractive index $\Delta n(\vec{E}) \sim \vec{E}$ is known as the linear electro-optic effect or Pockels effect. $\Delta n$ is almost independent of the wavelength and the processes involved are much faster than the electric field can be applied.

Electro-optic beam deflectors utilize the Pockels effect to create a linear refractive index gradient perpendicular to the direction of beam propagation, resulting in a deflection of the beam (Figure 4.2b): When passing the crystal, the light wave at the position $r$ from the beam center propagates with a speed of

$$
c(r)=\frac{1}{\left(n_{0}+\Delta n(r)\right)} c_{0} \approx \frac{1}{n_{0}}\left(1-\Delta n(r) / n_{0}\right) c_{0}
$$

where $n_{0}$ is the refractive index at $E=0, \Delta n$ the change induced by the electric field at position $r$ and $c_{0}$ the speed of light in vacuum. The approximation is valid for small $\Delta n$. The following calculations will also assume a small total deflection angle.

The difference in propagation speed will cause a deflection of the beam: With $\vec{z}$ as initial beam propagation direction, $\vec{x}$ the direction of the refractive index gradient and $n^{\prime}=d n / d x \sim$ $d \vec{E} / d \vec{r}$, the deflection angle $\beta^{\dagger}$ is:

$$
\mathrm{d} \beta^{\dagger}=\frac{n^{\prime}}{n} \mathrm{~d} z \quad \Rightarrow \quad \beta^{\dagger}(z)=\frac{n^{\prime}}{n_{0}} z
$$

When the beam exits the deflector it is additionally refracted at the crystal-air interface. The total deflection angle $\beta$ in a crystal with length $l$ is: 


$$
\frac{\sin \beta}{\sin \beta^{\dagger}}=n \Rightarrow \beta \approx n_{0} * \beta^{\dagger}=n^{\prime} l
$$

The total deflection depends linearly on the refractive index gradient $n^{\prime}$, which is proportional to the applied voltage gradient. The displacement $X$ of the beam is

$$
\mathrm{d} X=\beta^{\dagger}(z) \mathrm{d} z \quad \Rightarrow \quad X(l)=\frac{n^{\prime}}{2 n_{0}} l^{2}
$$

The displacement has to be considered when choosing the beam width as it reduces the usable aperture of the deflector.

For optimal beam scanning performance, the virtual point of beam deflection, a so called pivot point, must not depend on the deflection angle itself. Tracing back the deflected beams trajectory leads to:

$$
P_{z} \approx l-\frac{X}{\beta}=l\left(1-\frac{1}{2 n_{0}}\right)
$$

Projecting the pivot point onto the objective's back focal plane with a telescope will allow beam deflection without causing a beam displacement.

The linear gradient of the electric field is usually generated by contacting the crystal with four electrodes parallel to the beam propagation axis [71, p. $603 \mathrm{ff}]$.

\section{Application}

The following points need to be considered when choosing a deflector for the scanning system:

1. The crystal material defines the basic properties of the deflector (e.g. optical quality, damage thresholds, strength of the Pockels effect)

2. The dimensions of the crystal will limit the performance:

a) A small diameter allows higher field gradients at the same applied voltage, hence more deflection. However, the beam eventually has to fill the objective's back focal plane. Widening the beam diameter by $x$ reduces the deflection angle by $1 / x$.

b) The deflection angle is proportional to the crystal length. Yet the displacement of the beam scales with $l^{2}$ and the maximum deflection angle will be limited by the beam displacement.

3. The bandwidth and maximum current of the voltage drivers are the only limiting factors for the dynamic performance. The deflectors act as capacitors, requiring the drivers to provide a current $I \sim \mathrm{d} V / \mathrm{d} t$ when changing the voltage.

Crystal material: There are a variety of electro-optic crystals. The materials used for deflectors exhibit the electro-optic effect only for one polarization, which is a drawback as the non-polarized fluorescence signal cannot be completely de-scanned for confocal detection. No material was found to excel in a relevant property. However lithium niobate was discarded due to its low photodamage threshold (personal communication with Leysop). In 
some materials (e.g. Ammonium Dihydrogen Phosphate, ADP) the electro-optic effect can be increased by replacing the hydrogen with deuterium.

Crystal dimension: Homogeneous illumination of the objective's back aperture is essential for a good image quality. The beam width at the objective should therefore be larger than the aperture $2 r_{O}$. In case of a small beam width, the beam has to be widened with a telescope (see also section 4.1.3 and Figure 4.3) where the magnification is given by

$$
m=\frac{r_{O}}{r_{B}^{D}}=\frac{f_{2}}{f_{1}}
$$

with $r_{B}^{\mathrm{D}}$ : Beam size at deflector; $f_{2}, f_{1}$ : focal lengths of the telescope lenses (beam passes lens 1 first).

This also reduces the deflection angle at the objective:

$$
\alpha=\frac{\beta}{m}
$$

The usable beam size at the deflector is limited by the deflector aperture $D$ and the dislocation $X$. With equations (4.2), (4.3) and (4.4):

$$
r_{b}^{\max }=D / 2-X^{\max }=D / 2-\frac{\beta^{\max } l}{2 n_{0}}
$$

With the above equations the maximal deflection angle at the objective can be written as:

$$
\alpha^{\max }=-\left(\beta^{\max }\right)^{2} \frac{l}{2 r_{O} n_{0}}+\beta^{\max } \frac{D}{2 r_{O}}
$$

Solving for the maximum $\alpha$ yields:

$$
\alpha^{\max }=\frac{D^{2} n_{0}}{8 l r_{O}}
$$

Voltage Drivers: Equation (4.7) defines the maximum deflection angle at the objective using a crystal with diameter $D$ and length $l$. Achieving this angle requires a sufficient voltage $U$ to create the refractive index gradient. Generating high voltages is not a big challenge and as no current flow is required a static deflection is easily accomplished. However, as the goal is to scan the beam as fast as possible, a suitable voltage driver also needs to be able to deliver the necessary current $I$ :

$$
C=\frac{\mathrm{d} Q}{\mathrm{~d} U}=\frac{I \mathrm{~d} t}{\mathrm{~d} U} \Rightarrow I=C \frac{\mathrm{d} U}{\mathrm{~d} t}
$$

with $C$ being the capacitance of the scanning system. The higher the maximal available voltage, the more difficult it is to design a driver with the desired dynamic properties.

It was not in the scope of this thesis to design the electro-optic deflectors or voltage drivers. Commercially available devices were compared and selected to best fit the mentioned criteria. The used deflectors are manufactured from a lithium tantalate crystal (model ED3, 
Leysop, Essex, England) and ammonium dideuterium phosphate (customized model M311A, Conoptics, Danbury, CT). The voltage drivers (models 2220-CE and PZD700A-1-HSHV-CE by Trek, Medina, NY) were selected to match the damage threshold of the scanners and to provide reasonable dynamic performance. The details of the scanning system properties and results of above calculations is presented in Table 4.1. The scanning system with the Leysop deflector was used as the slow scanning axis, as its scanning speed is considerably lower compared to the conoptics scanner. However, I would like to point out that so far, none of the experiments carried out would have been limited by the properties of either deflection system.

\begin{tabular}{|c|c|c|c|}
\hline \multicolumn{4}{|c|}{ Deflector Properties } \\
\hline Deflector & Leysop ED-3 & Conoptics M-311A & \\
\hline Crystal material & lithium tantalate & $\mathrm{AD}^{\star} \mathrm{P}$ & \\
\hline Aperture diameter $D$ & 3 & 2.5 & $\mathrm{~mm}$ \\
\hline Length $l$ & 25 & 200 & $\mathrm{~mm}$ \\
\hline$n_{0}$ & 2.18 & 1.54 & \\
\hline Deflection & $\perp$ polarization & \|polarization & \\
\hline Capacitance $C$ & 180 & 50 & $\mathrm{pF}$ \\
\hline Deflection factor $c$ & 1.5 & 7.1 & $\mu \mathrm{rad} / \mathrm{V}$ \\
\hline Max. voltage & 2000 & 500 & V \\
\hline \multicolumn{4}{|c|}{ Voltage Driver } \\
\hline Model & 2220-CE & PZD700A & \\
\hline Voltage range & \pm 2000 & \pm 700 & V \\
\hline Bandwidth & $>7.5$ & $>125$ & $\mathrm{kHz}$ \\
\hline Max. current & \pm 10 & \pm 100 & $\mathrm{~mA}$ \\
\hline \multicolumn{4}{|c|}{ Final parameters } \\
\hline$\alpha^{\max }$ & \pm 0.75 & \pm 1.25 & $\mu \mathrm{rad}$ \\
\hline Max. scan speed & 0.075 & 1.5 & $\mu \mathrm{m} / \mu \mathrm{s}$ \\
\hline Max. scan range (theory) & \pm 1.5 & \pm 2.5 & $\mu \mathrm{m}$ \\
\hline Max. scan range (experiment) & \pm 1.75 & \pm 2.375 & $\mu \mathrm{m}$ \\
\hline
\end{tabular}

Table 4.1. Properties of the electro-optical scanning system. The final scan parameters were calculated with the equations in the main text, regarding the experimental implementation $\left(m=4, f_{\mathrm{O}}=2 \mathrm{~mm}, C_{\text {wires }}=50 \mathrm{pF}\right)$. The system was experimentally calibrated on a fluorescent grating with $140 \mathrm{~nm}$ line distance. The maximum voltage given is the limit recommended by the manufacturer and was exceeded for the Conoptics scanner. AD*P: Ammonium dideuterium phosphate 
As the field of view attainable with electro-optic scanning is very limited, a piezo-driven sample stage was used for large FOVs.

\subsubsection{The Setup}

The STED laser used is the same as for the two-color setup (section 3.1), as are the basic design principles for a STED microscope. Recurring components such as filters, waveplate or the objective serve the same purpose and were selected following the same considerations. Most changes are due to the implementation of the electro-optic scanning system. To reduce complexity, it features only one excitation laser at $635 \mathrm{~nm}$ (PicoQuant, Berlin) and one fluorescence detection channel from $645-725 \mathrm{~nm}$, optimized for the far-red dyes used in the two-color setup. As described below, two detectors are necessary to collect all fluorescence signal. A time gate processes the detector signal before it is sent to the data acquisition card. The microscope setup is sketched in Figure 4.3, the components are listed in Table 4.2.

The operating principle of beam scanning and electro-optic deflectors is outlined in Figure 4.2. Three telescopes were used to adjust the beam size and project the pivot points of the scanners (P1, P2) on the objective's back focal plane. When passing the vortex phase plate the beam should be several millimeters in diameter to keep imperfections and interference close to the vortex singularity at a minimum. The beam size has to be reduced by the telescope lenses TL1, TL1' before passing the electro-optic deflectors due to their small aperture. A second telescope (TL2, TL2') projects P1 on P2. A third telescope (TL3, TL3') widens the beam to fill the objective's back aperture and projects the pivot points to its back focal plane. All telescopes are built of two positive aspherical lenses. Because the lens on the deflector side needs to have its focal point at the deflector's pivot point, the length of the deflector limits the minimum focal length. The focal length of the second lens is defined by the desired magnification of the telescope. Solving equations (4.5) and (4.6) for the optimal beam diameter yields:

$$
r_{B}^{\mathrm{opt}}=D / 4
$$

The beam in the Conoptics deflector $(D=2.5 \mathrm{~mm})$ should therefore have a width of $1.25 \mathrm{~mm}$ to achieve the maximum possible scan range. As the maximal applied voltage is only $700 \mathrm{~V}$ the beam width can be up to $1.84 \mathrm{~mm}$ before it is clipped by the deflector's aperture. The objective's back aperture is $5.6 \mathrm{~mm}$ wide, the magnification of the last telescope should therefore be $>$ 3.1. In order to ensure a good illumination of the objective's aperture, the final telescope widens the beam 4 -fold. The resulting scan range is $\approx 5 \mu \mathrm{m}$ with the Conoptics scanner and $\approx 3 \mu \mathrm{m}$ with the Leysop scanner.

The Leysop deflector introduced strong astigmatism that had to be corrected. This is done by two pairs of cylinder lenses with focal lengths $f=500 \mathrm{~mm}$ (CL1', CL2) and $f=-400 \mathrm{~mm}$ (CL1, CL2'). Two pairs are necessary as the first widens the beam in one direction by 1/4. When the STED beam is focused by the objective, the electro-magnetic field components at $0^{\circ}$ and $180^{\circ}$ of the beam interfere in the focus center resulting in a $\mathrm{z}$-directed field. The field at $90^{\circ}$ and $270^{\circ}$ interfere to a $180^{\circ}$ phase shifted field, thus annihilating the $z$-component resulting in an intensity-zero (Figure 1.4). If the beam's intensity distribution does not exhibit this $90^{\circ}$ symmetry, the residual $\mathrm{z}$-component will quench the fluorescence in the doughnut center, reducing the available signal and limiting the resolution. The second cylinder lens 


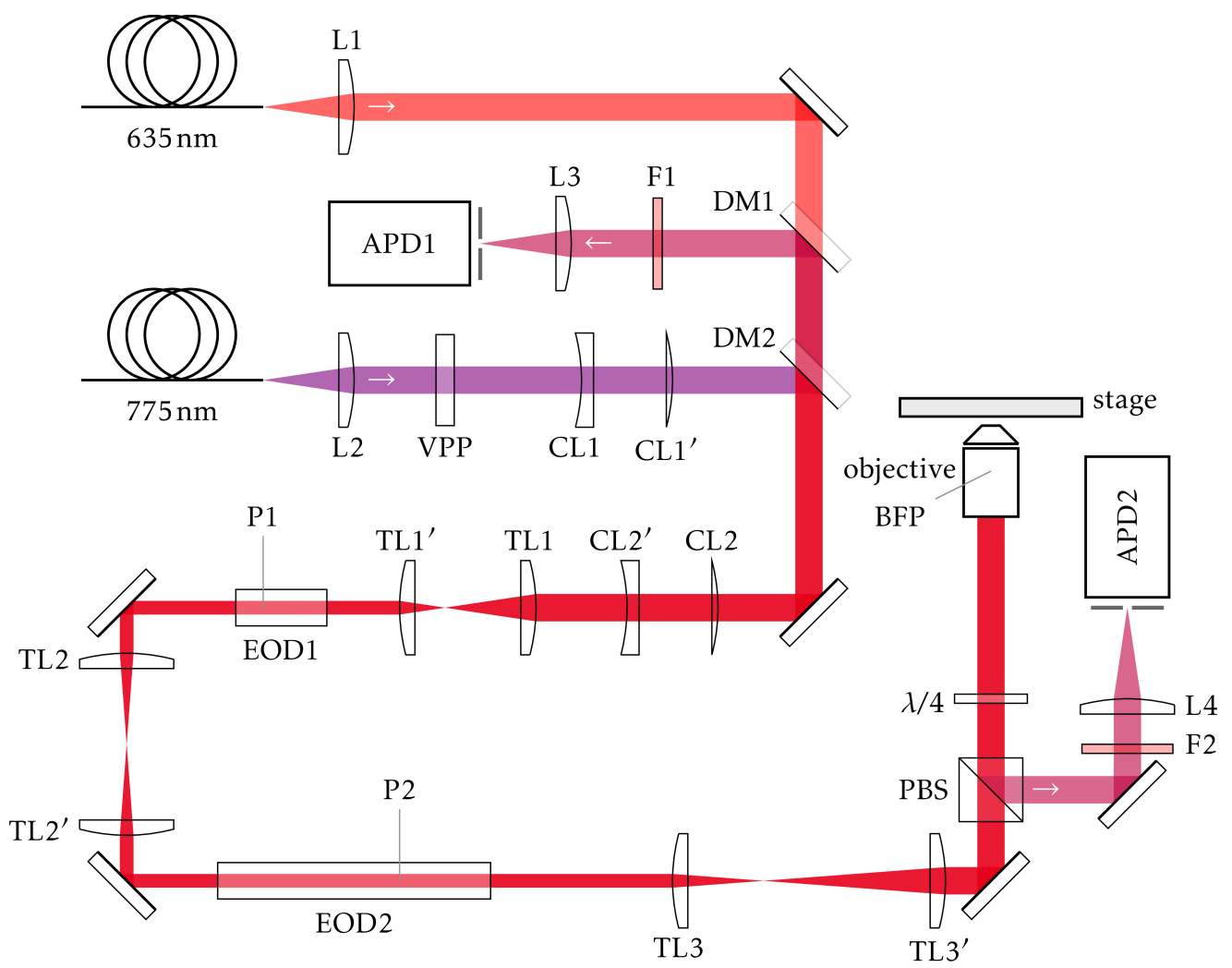

Figure 4.3. Outline of the small area STED setup. The STED laser at $775 \mathrm{~nm}$ is the same as used in the two-color STED microscope, the excitation wavelength is $635 \mathrm{~nm}$. The laser beams exit from fibers and are collimated with lenses L1, L2. STED, excitation and APD1-detection path are aligned with DM1, DM2 and coupled into the electro-optic scanning system: A telescope (lenses TL1, TL1') reduces the beam diameter, the beams then pass the first electro-optic deflector (EOD1), deflecting in y-direction. A second telescope (TL2, TL2') projects the pivot point P1 of EOD1 on the pivot point of the second deflector P2. EOD2 deflects in $\mathrm{x}$-direction, its pivot point is projected (lenses TL3, TL3') on the objective's back focal plane (BFP). A vortex phase plate (VPP) imprints the $2 \pi$ phase pattern onto the STED wavefront. A pair of cylinder lenses $\left(\mathbf{C L 1}, \mathbf{C L 1} \mathbf{1}^{\prime}\right)$ is used to correct for astigmatism but stretches the beam in one direction. This is compensated with a second, identical pair (CL2,CL2'). The polarization of the beams is cleaned with a polarizing beam splitter (PBS) after the scanning system. A $\lambda / 4$ waveplate circularizes the STED beam's polarization before the laser beams enter the objective. For overview measurements the sample is moved with a piezo stage. Only one polarization direction of the fluorescence signal is transmitted by the PBS. In order to maximize the signal a second detector (APD2) detects the reflected fluorescence. The fluorescence signal is filtered for wavelength with filters $\mathbf{F} 1$ and $\mathbf{F} 2$ to remove scattered and reflected laser light. The detector signal is time gated (see section 3.1). 


\begin{tabular}{|lrr|}
\hline L1 & achromat & $f=60 \mathrm{~mm}$ \\
L2 & achromat & $f=25 \mathrm{~mm}$ \\
L3, L4 & achromat & $f=200 \mathrm{~mm}$ \\
TL1 & achromat & $f=180 \mathrm{~mm}$ \\
TL1' & achromat & $f=50 \mathrm{~mm}$ \\
TL2, TL2' & achromat & $f=200 \mathrm{~mm}$ \\
TL3 & achromat & $f=100 \mathrm{~mm}$ \\
TL3' & achromat & $f=400 \mathrm{~mm}$ \\
CL1, CL2' & cylinder lens, & $f=-400 \mathrm{~mm}$ \\
CL1', CL2 & cylinder lens, & $f=500 \mathrm{~mm}$ \\
DM1 & & Z650SPRDC \\
DM2 & & Z770RDC \\
F1, F2 & & $685 / 80 \mathrm{ET}$ \\
$\lambda / 4$ & 600-900 nm achromat \\
Objective & PL APO 100x 1.4-0.7 Oil \\
\hline
\end{tabular}

Table 4.2. List of main optical components. DM1, DM2, F1 and F2 manufactured by Chroma Technology, Bellows Falls/VT; objective by Leica Microsystems, Wetzlar; $\lambda / 4$ by B. Halle Nachfl., Berlin

pair (CL2, CL2') compensates the beam distortion by the first pair. The excitation and detection are not as sensitive to the distortion. Positioning the first CL-pair (CL1, CL1') in the STED-only beam path allows a more independent astigmatism correction for the excitation and STED beams.

The electro-optic crystals deflect only one polarization direction and are birefringent. If the polarization is not perfectly aligned to the deflection axis, part of the beam will pass undeflected and the polarization might be rotated. Both will result in a suboptimal intensity-zero of the STED-doughnut. The polarization is therefore cleaned with a polarizing beam splitter after the deflectors. The fluorescence signal emerging from the objective is split at the same beam splitter, transmitted fluorescence is de-scanned by the deflectors and detected by a fiber coupled APD. De-scanning is important as the fiber core acts as a confocal pinhole. The reflected, not de-scanned part of the fluorescence signal is also detected confocally by a fiber coupled APD. However, most of the signal will be blocked by the pinhole/fiber core for scan positions $>100 \mathrm{~nm}$ from the center, which is not an issue for small scan fields. The pinholes are 1.1 Airy disk in size.

Overview images are acquired with a piezo driven stage (P-562.3CD with E712-driver, Physik Instrumente, Karlsruhe, Germany) which allows large scan fields up to $200 \mu \mathrm{m}$ in $\mathrm{x}, \mathrm{y}$ and $\mathrm{z}$ directions.

\subsubsection{Software}

\section{Motivation}

Software is used to simplify the control of the microscope's hardware, automatize repetitive tasks and present data in a user-readable way. While the software calculates the parameters and may interfere with a running measurement, the actual measurement is still controlled 


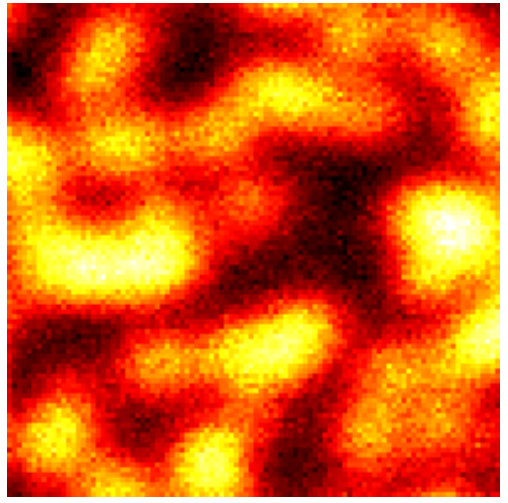

(a) confocal

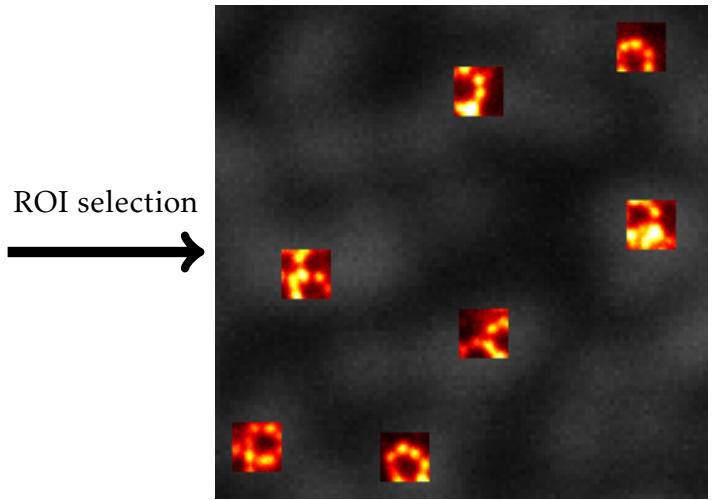

(b) STED composite

Figure 4.4. Confocal image (a) and a composition of several STED scans (b), displayed at their position in the confocal image. The software needs to provide functions to select regions of interest (ROIs) in the overview scan, which are then automatically scanned.

by the hardware components and written into the PCs memory by Direct Memory Access (DMA). The developed STED microscopes acquire an image by scanning the sample or the laser foci and measuring the fluorescence signal collected by the detectors. In order to visualize the investigated structure, the collected signal has to be binned into pixels representing its origin in the sample. The main task of the measurement software is to calculate the scanning pattern and correlate the measured signal with the scan position. The detailed implementation depends on the used hardware. Here the data acquisition (DAQ) module NI PCIe-6353 (National Instruments) was used to acquire the detector signal and control the scanner movement. Figure 4.5 shows the main components of the device.

The analog output and input channels are used to control the positions of the scanners and measure analog sensor signals, the digital outputs to switch laser shutters and other binary components, the digital input to read slow digital signals and the counter input channels to count the number of voltage pulses (e.g. from an avalanche photo diode) in a certain time interval. The various channel types can be configured and synchronized mostly independently. Once configured by the software, the signal generation, detection and synchronization is controlled by the DAQ module. This ensures a high temporal resolution and fast response to conditional events.

For measurements on the two-color STED setup the software Imspector [59] was used. It supports many devices and provides a comprehensive graphical user interface including data analysis and manipulation tools.

Scanning small regions, however, requires some features that Imspector did not provide:

- the region to be scanned should be easily selectable (Figure 4.4)

- shifts between measured and actual position should automatically be compensated

- automatic region selection by brightness or shape for well defined samples to achieve a high throughput

- manual region selection directly in the overview image 


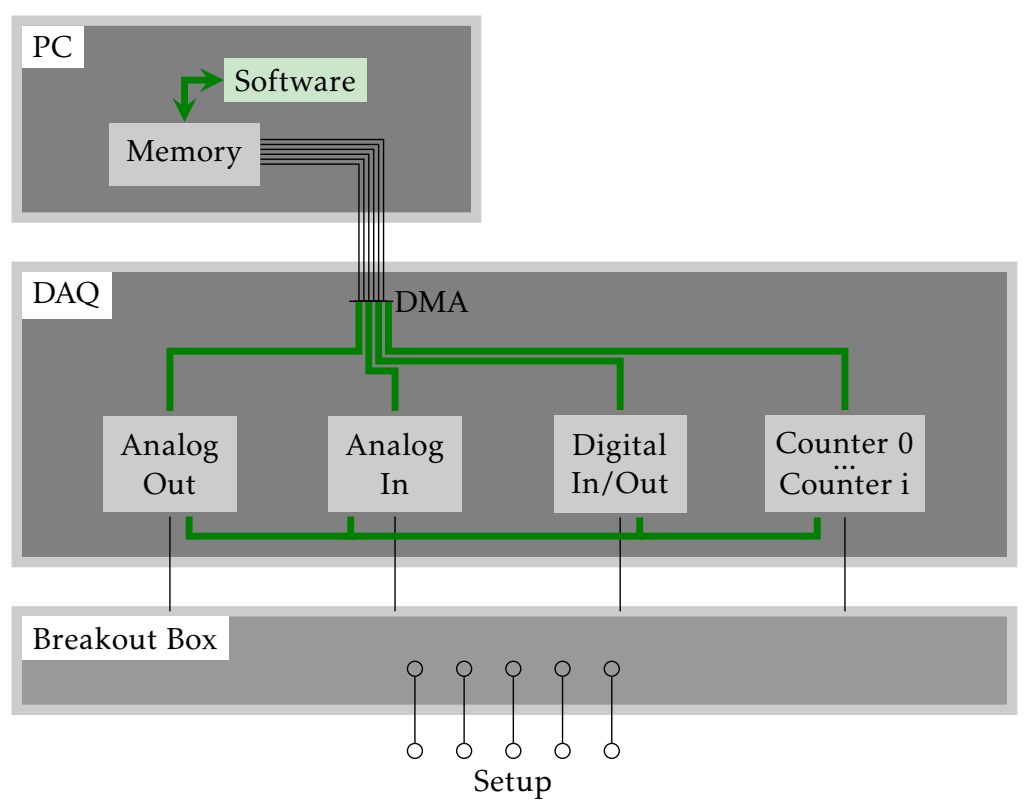

Figure 4.5. Schematic of the measurement control hardware. The data acquisition (DAQ) card is connected to the PC via a PCIe bus. The DAQ card has modules for analog output voltage generation, voltage measurement, digital input/output and counter input/output channels. The channels are connected to the Breakout Box where they can be accessed and connected to the setup. The configuration and interconnection of the hardware modules are flexible and set by the software (green connections).

- a measurement set will consist of many (>10) small area scans that need to be organized and be easily accessible

- a defined frame rate for time resolved measurements, i.e. the back scan needs to be included in the image

- the analog output sample rate may need to be higher than the pixel dwell time since the voltage driver of the scanner overshoots when discreet voltage steps occur

- new hardware components not supported by Imspector may be required

Some of the mentioned problems can be solved with the Python scripting interface to Imspector. The scripting interface, however, does not provide access to the deeper functionality such as the scanning pattern or organization of windows. It was therefore necessary to write custom scanning routines and later to develop a graphical user interface. Presently, the program is an independent measuring software that is straightforward to use and provides high flexibility when creating custom measurements.

Python 2.7 [72] was chosen as the programming language. It is a general-purpose scripting language that is widely used in science and offers a broad set of libraries. Writing a working program is faster and easier compared to $\mathrm{C}^{++}$while offering more functionality than MATLAB or LabVIEW. The reference implementation CPython is open source, which eases the future reuse of the program. 


\section{Scanning Field of Views Below the Diffraction Limit}

\section{Program Structure}

The structure of the program was designed with the intention of creating a modular base that can be easily reused and modified by anyone with basic programming skills. Functions and objects are combined in files according to their purpose and dependencies. The most important ones are outlined in Figure 4.6, some shall be described here briefly. A more detailed documentation is included in the program and will be available on the project site.

Measurement objects provide a set of methods to access data, configure and start a measurement. There are several instances and subclasses that introduce the functionality stepwise: A base_measurement class defines the structure of a measurement with methods to add, delete and access data stacks. When loading a measurement from file, a base_measurement object is created. The scan_measurement inherits the base_measurement object and adds functions to set up a common configuration for a measurement, e.g. scan range or pixel size. The nidaq_measurement can start a measurement. It inherits the scan_measurement object and adds the functions necessary to convert the scan configuration to hardware-specific scan parameters.

This object-oriented approach ensures that the most basic methods are included in any derived measurement object while more hardware specific methods may differ (e.g. different number of analog output channels). Also, adding a new function to the base_measurement instantly makes this function available to all measurements. Depending on the specific problem, a measurement object may be further sub-classed in Templates where certain measurement parameters are automatically set and new functions may be implemented.

The configurations object is the central object for storing information on the setup and measurement parameters. The parameters are stored in dictionaries as pairs of keys and values. This allows a human readable and organized structure. There are three main types of parameter dictionaries: The hardware configuration is stored in a file and should not be edited by individual measurements. It provides information on the scan range limits of the scanners, voltage limits etc. The scan configurations include the measurement specific parameters such as pixel size, dwell time and scan axes. The default values may be stored in the same file as the hardware configurations but should be set specifically by the user or the template files. Combining the hardware and scan configurations the hardware-specific measurement object creates the scan parameters. The scan parameters are less abstract and contain direct, hardware-related information (e.g. voltage range instead of physical scan range).

All configuration objects are stored with the measurement.

Hardware control objects configure the hardware to perform the actual measurement. A central object configures the individual signal generation and detection devices and creates the routing for synchronization. Separating the hardware control from the measurement object reduces the complexity for the user while maintaining a high flexibility in the implementation of the measurement procedure.

libnidaqmx is an open source Python wrapper for the NI-DAQmx DLL [73]. It was slightly modified. The NI-DAQmx DLL is provided by National Instruments and exposes the interface to configure the data acquisition card. 


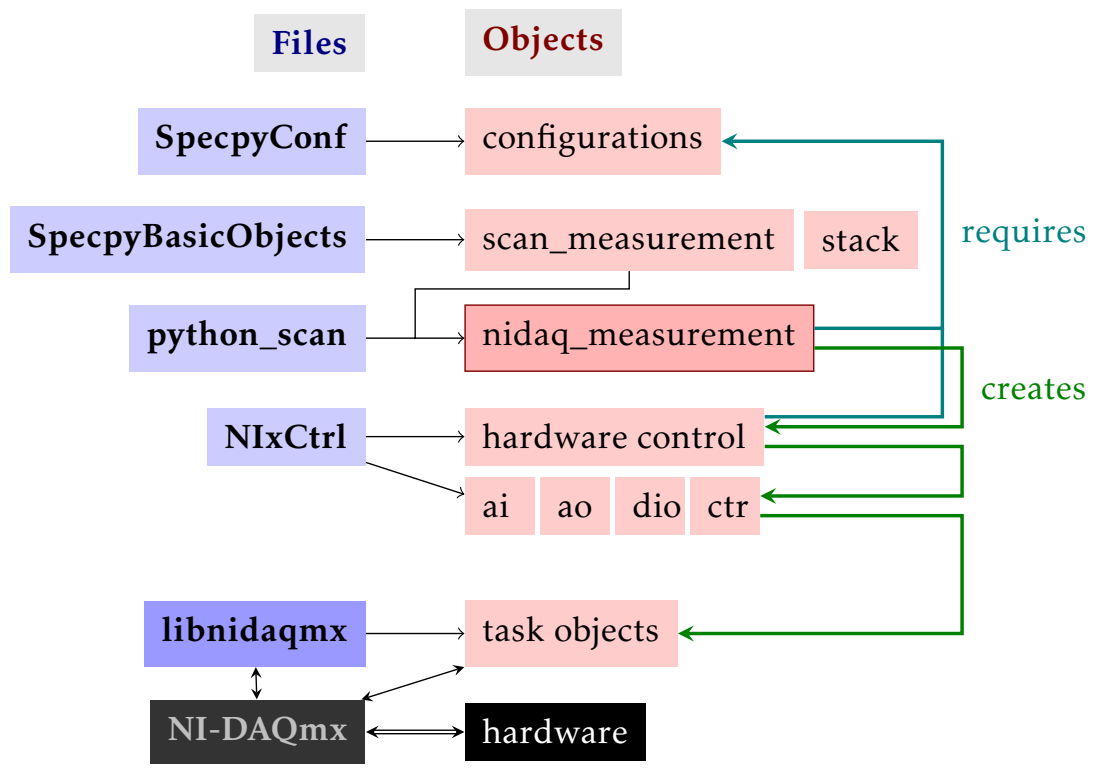

Figure 4.6. Overview of the most important files (blue) and objects (red) of the program. Details are in the main text.

The measurement data is stored in the HDF5 [74] format. HDF5 provides a flexible and open data model that allows an easy addition of new data and parameter sets as the application evolves without sacrificing backwards compatibility. Application programming interfaces for several programming languages including Python are provided by the HDF group. HDF5 files can be read by any HDF viewer, ensuring the future accessibility of the data.

\section{The Graphical User Interface}

The measurement components of the program were designed to operate independently from a graphical user interface (GUI). During the early development, Imspector was used via its Python scripting interface to display data and set basic measurement parameters. However, with increasing complexity of the project, a more specialized GUI became necessary. Qt 4.8 [75] and its Python binding PySide [76] were used to develop the GUI. Qt is a cross platform application framework with excellent documentation that allows efficient development of GUI applications.

The goal was not to build a comprehensive measurement and analysis application but to provide a clean, easily extendable interface to the measurement. The GUI provides standard features such as controls for the scanner position, editing of measurement parameters and data visualization. The measurement data can be exported as OME Tiff [77, 78] file for data analysis with compatible programs. Qt Widgets can be added easily as a plugin for specialized applications. The coordination and automation of small area measurements is implemented as a plugin. 


\section{Application example: Measuring single molecule bleaching rates}

The operation of the program shall be exemplified on the measurement procedure for the bleaching experiments described in section 4.2. The experimental setup and sample preparation are described in sections 4.1.3 and 2. A single molecule bleaches with a certain probability $p$ per time interval. As only the time until the molecule bleaches is experimentally accessible, many molecules have to be measured to get an approximation for $p$. The acquisition of a single data point requires averaging over $\approx 100$ measurements. Therefore the measurement process is fully automated ${ }^{1}$.

1| An overview image is acquired. The positions in the overview image have to be very accurately matched to the real positions in the sample as they will have to be approached again later. The two main causes for inaccuracy are the inertia of the stage and position sensor lag.

The scan waveform generation is carried out by the piezo stage driver, which also provides the pixel clock. It features a Digital Dynamic Linearization algorithm that automatically corrects differences between the set and actual position of the stage. However, this feature proved to be unreliable and caused oscillations. It was therefore not used. Instead, the stage position during a scan of 5 line cycles was recorded and the region with the best linear approximation used for signal acquisition. The position error was stored in the measurement object to compensate for when moving to a specified position ${ }^{2}$. Typically, the deviation from linearity was $\pm 0.8 \mu \mathrm{m}$ for a scan length of $50 \mu \mathrm{m}$ with $50 \mathrm{~nm}$ pixel size and $100 \mu \mathrm{s}$ dwell time. After the calibration, the regular scan was executed and the overview image acquired.

2| The molecules in the overview image are identified. The overview image was smoothed with a Gaussian filter in order to get accurate positions, reducing the inherent noise of the low fluorescence signal. Then the local maxima with an intensity above a given threshold were identified as molecule positions. To avoid bleaching one molecule of interest when measuring another, only positions further apart than $1 \mu \mathrm{m}$ are scheduled for measurement.

3| The molecule's position is approached. After moving the piezo stage to the molecule's position, a confocal electro-optic scan of $2 \mu \mathrm{m} \times 2 \mu \mathrm{m}$ area is executed. The measured signal is correlated with the signal of the overview scan to correct the stage position. This is necessary due to the time lag of the piezo stage's position sensors $(\approx 1 \mathrm{~ms})$ and sample drift. Optionally, a 3D image stack is acquired to correct the drift out of the focal plane. After correcting the stage position, another confocal scan is acquired. The second scan serves as a control for the actual stage position when analyzing the data. Additionally the position of the molecule is determined more accurately on the second scan.

4| The molecule's bleaching time is measured. The laser focus is moved to the molecule's position with the electro-optic scanners. As the STED laser inhibits the fluorescence, a mechanical chopper is used to periodically block it. Each molecule is measured for $20 \mathrm{~s}$. With a mean bleaching time of $\approx 2.5 \mathrm{~s}$, most molecules bleach within this time interval. The chop-

\footnotetext{
${ }^{1}$ Implemented in the file python_scan_GUI/Plugins/SmallScan/smallScan0b jects.py

${ }^{2}$ Details in the files PICtrl.py and Templates/pi_scan_accurate.py
} 
per signal is monitored in synchrony with the fluorescence to separate the two signals (see section 4.2.3). The measurement continues for the next molecule position with step 3.

\subsection{Bleaching in STED Microscopy}

\subsubsection{Considerations}

The goal of the following experiments was to measure the bleaching rate of a dye as a function of the applied excitation and STED power. In order to provide well defined experimental conditions with a minimum of possible error sources the following points were considered:

- The laser intensity at the probe should be known and constant. Scanning the sample should be avoided, as each fluorophore receives a varying irradiation when the laser focus passes. The same argument dismisses probes extending over several $10 \mathrm{~nm}$, or dense samples.

- The photophysical properties of fluorescent dyes are extremely sensitive to their environment. The sample buffer should therefore be well defined and stable.

- Bleaching usually is considered to be a memoryless probabilistic process, i.e. the probability that a molecule under irradiation bleaches within a certain time is constant. The temporal evolution of the fluorescence signal therefore resembles an exponential decay. Not all fluorophores follow this model [79]. For example, long-lived photoinduced dark states or fluorescence quenching in dense samples cause deviations from the exponential bleaching behaviour.

In short: The sample should be made of sparsely distributed small $(\approx 10 \mathrm{~nm})$ fluorescent particles, possess long-term stability, reproducibility, and typical bleaching behaviour.

A long bleaching time is desired as it increases the signal-to-noise ratio of the measurements. An optimized mounting medium can increase the dye's lifetime by orders of magnitude [53]. Yet these media degrade with time $(\approx$ days) and are difficult to prepare with the necessary reproducibility for these experiments.

Considering these facts and testing various samples and buffers, immobilized single Atto647N molecules in phosphate-buffered saline (PBS) were chosen for the bleaching measurements. The samples proved to be long-term stable (several weeks) and reproducible to prepare. The sample preparation is described in Chapter 2

\section{Laser Power and Intensities}

While the experimenter measures the power of the lasers in the setup, the more relevant figure is the intensity in the sample. However, it is hard to intuitively compare intensities in the order $\mathrm{MW} \mathrm{cm}^{-2}$, even more so when the peak intensity of a laser pulse is given. Additionally, the model considers only $\mathrm{CW}$ illumination. Therefore, the time-averaged power and intensities are used when fitting the model to the data.

Absorption cross sections $\sigma$ have the unit $\left[\mathrm{cm}^{2}\right]$, the measured laser power is therefore converted to the photon flux through the focus with the unit [photons $/ \mathrm{cm}^{2} \mathrm{~s}$ ]. Assuming a Gaussian intensity distribution with a FWHM of $250 \mathrm{~nm}$ and $300 \mathrm{~nm}$ for the excitation and STED focus, the relation between power and maximum intensity is summarized in Table 4.3. 


\begin{tabular}{|lccc|}
\hline & excitation & STED & unit \\
Power & 1 & 1 & W \\
FWHM & 250 & 300 & $\mathrm{~nm}$ \\
Intensity & $1.4 \cdot 10^{9}$ & $0.98 \cdot 10^{9}$ & $\mathrm{~W} \mathrm{~cm}^{-2}$ \\
Photon flux & $4.5 \cdot 10^{27}$ & $3.8 \cdot 10^{27}$ & $\mathrm{~cm}^{-2} \mathrm{~s}^{-1}$ \\
\hline
\end{tabular}

Table 4.3. Conversion between time averaged power and intensity. Intensity is calculated at the peak of a Gaussian with according FWHM. An additional factor of 0.42 for the excitation and 0.56 for the STED intensities has to be taken into account when converting the power values given in the graphs.

Within the current section 4.2, the power indicated is the laser power measured in the setup, after the second electro-optic deflector. Of the power indicated, $80 \%$ of the STED and $60 \%$ of the excitation power enter the objective's back aperture. Approximately $70 \%$ of the laser power entering the objective is deposited in the sample. For the sake of using round numbers, these factors were not applied to the indicated power. The fit results in Table 4.4 include these correction factors.

\subsubsection{Modeling the Bleaching Behavior}

The derivation below roughly follows the argumentation in [80]. Photobleaching is a very complex process that may involve intermediate and competing molecular transitions. In the simplest case, the molecule bleaches with a constant probability $p_{\text {bleach }}$ per time interval $\Delta t$ of irradiation. Therefore, the integrated signal intensity $I$ over many molecules is described by an exponential decay:

$$
I(t)=I_{0} \exp \left(-r_{\text {bleach }} t\right)+y_{\text {bg }}
$$

with the initial intensity $I_{0}$, the bleaching rate $r_{\text {bleach }}$ and a background term $y_{\text {bg }}$ that originates from experimental limitations such as detector noise.

As photobleaching involves the excitation of the fluorescent molecule, it should occur from excited electronic states $S_{i}$ and $T_{i}$. The rate of photobleaching from a particular state is

$$
r_{\text {bleach }}^{(\text {state }}=k_{\text {bleach }}^{(\text {state) }} p^{(\text {state })}
$$

where $k_{\text {bleach }}^{\text {(state }}, p^{\text {(state) }}$ is the bleaching rate constant and the occupation probability of the state. In the following paragraphs, $r, k$ and $p$ will denote rates, rate constants and occupation probabilities respectively. Subscripts indicate the transition and superscripts the multiplicity or states involved (e.g. $k_{1 n}^{(\mathrm{S})}$ is the rate constant of the transition $\mathrm{S}_{1} \rightarrow \mathrm{S}_{n}$ ).

The different time scales of processes involved in photobleaching (electronic and molecular transitions: fs-ps; electronic state lifetimes: ns; bleaching: s) allow to handle them separately. In the following model, state transitions are assumed to be instantaneous and bleaching too slow to reduce the dye population.

First, a simple two-level system under $\mathrm{CW}$ irradiation shall be considered. According to (4.9), the bleaching rate would be proportional to the probability $p^{\left(S_{1}\right)}$ of the $S_{1}$ state being 
occupied. With the rate constants for excitation and relaxation $k_{01}=\sigma_{01} I_{\text {exc }}$ and $k_{10}=1 / \tau_{\mathrm{S}_{1}}$, we get the differential equation:

$$
\begin{aligned}
\frac{d p^{\left(\mathrm{S}_{1}\right)}}{d t} & =k_{01}\left(1-p^{\left(\mathrm{S}_{1}\right)}\right)-k_{10} p^{\left(\mathrm{S}_{1}\right)} \\
\Rightarrow \quad p^{\left(\mathrm{S}_{1}\right)}(t) & =\left.p^{\left(\mathrm{S}_{1}\right)}\right|_{t=0} \exp \left(-\left(k_{10}+k_{01}\right) t\right)+\frac{k_{01}}{k_{01}+k_{10}}
\end{aligned}
$$

For the equilibrium case this expression simplifies to:

$$
p^{\left(\mathrm{S}_{1}\right)}=\frac{k_{01}}{k_{01}+k_{10}} \quad \stackrel{k_{01} \ll k_{10}}{\approx} \quad \frac{k_{01}}{k_{10}}=\tau_{\mathrm{S}_{1}} \sigma_{01} I_{\mathrm{exc}}
$$

and

$$
r_{\text {bleach }}=k_{\text {bleach }}^{\left(\mathrm{S}_{1}\right)} \cdot p^{\left(\mathrm{S}_{1}\right)} \approx k_{\text {bleach }}^{\left(\mathrm{S}_{1}\right)} \cdot \tau_{\mathrm{S}_{1}} \sigma_{01} I_{\text {exc }}
$$

i.e. the bleaching rate scales linear with the excitation power.

The relation $k_{01} \ll k_{10}$ is true for low excitation power, i.e. the fluorescence is not saturated and is proportional to the excitation intensity. This is mostly the case in relevant experimental conditions. Please note that the stimulated emission term, which usually needs to be considered in the rate equations, is much smaller than the other terms and was left out for simplicity. Including the contribution of the triplet state $\mathrm{T}_{1}$ in (4.13) does not qualitatively change the bleaching behavior, as the triplet state population is proportional to $p^{\left(\mathrm{S}_{1}\right)}$.

It is clear, however, that this simple model of bleaching will not suffice to describe STED conditions. The STED laser will cause a depopulation of the $S_{1}$ level and thus reduce the bleaching rate. This obviously contradicts the experimental experience.

Therefore, higher levels of excitation $S_{n}, T_{n}$ have to be taken into account. Further excitation $\mathrm{S}_{\mathrm{n}} \rightarrow \mathrm{S}_{\mathrm{m}}, \mathrm{T}_{\mathrm{n}} \rightarrow \mathrm{T}_{\mathrm{m}}$ and intersystem crossing $\mathrm{S}_{\mathrm{n}} \leftrightarrow \mathrm{T}_{\mathrm{m}}$ (ISC) is possible. However, the lifetimes of higher excited states are very low $(\approx$ ps $)$ so that ISC with $n>1$ is improbable and therefore neglected. Here, a 5-level system shall be considered. The bleaching rate is now the combination:

$$
r_{\text {bleach }}=k_{\text {bleach }}^{\left(\mathrm{S}_{1}\right)} \cdot p^{\left(\mathrm{S}_{1}\right)}+k_{\text {bleach }}^{\left(\mathrm{S}_{n}\right)} \cdot p^{\left(\mathrm{S}_{n}\right)}+k_{\text {bleach }}^{\left(\mathrm{T}_{1}\right)} \cdot p^{\left(\mathrm{T}_{1}\right)}+k_{\text {bleach }}^{\left(\mathrm{T}_{n}\right)} \cdot p^{\left(\mathrm{T}_{n}\right)}
$$

With these assumptions, one can write the differential equations in the matrix form ${ }^{3}$

$$
\frac{\mathrm{d}}{\mathrm{d} t}\left(\begin{array}{l}
p^{\left(\mathrm{S}_{0}\right)} \\
p^{\left(\mathrm{S}_{1}\right)} \\
p^{\left(\mathrm{S}_{n}\right)} \\
p^{\left(\mathrm{T}_{1}\right)} \\
p^{\left(\mathrm{T}_{n}\right)}
\end{array}\right)=\left(\begin{array}{ccccc}
-k_{01}^{(\mathrm{S})} & k_{10}^{(\mathrm{S})} & 0 & k_{10}^{(\mathrm{T} \rightarrow \mathrm{S})} & 0 \\
k_{01}^{(\mathrm{S})} & -k_{10}^{(\mathrm{S})}-k_{1 n}^{(\mathrm{S})}-k_{11}^{(\mathrm{S} \rightarrow \mathrm{T})} & k_{n 1}^{(\mathrm{S})} & 0 & 0 \\
0 & k_{1 n}^{(\mathrm{S})} & -k_{n 1}^{(\mathrm{S})} & 0 & 0 \\
0 & k_{11}^{(\mathrm{S} \rightarrow \mathrm{T})} & 0 & -k_{10}^{(\mathrm{T} \rightarrow \mathrm{S})}-k_{1 n}^{(\mathrm{T})} & k_{n 1}^{(\mathrm{T})} \\
0 & 0 & 0 & k_{1 n}^{(\mathrm{T})} & k_{n 1}^{(\mathrm{T})}
\end{array}\right)\left(\begin{array}{c}
p^{\left(\mathrm{S}_{0}\right)} \\
p^{\left(\mathrm{S}_{1}\right)} \\
p^{\left(\mathrm{S}_{n}\right)} \\
p^{\left(\mathrm{T}_{1}\right)} \\
p^{\left(\mathrm{T}_{n}\right)}
\end{array}\right)
$$

\footnotetext{
${ }^{3}$ Rate constants that depend on the STED or excitation intensity are printed red.
} 


\section{Scanning Field of Views Below the Diffraction Limit}

Solving this system under consideration of the steady state approximation $\left(\frac{\mathrm{d}}{\mathrm{d} t} \ldots=0\right)$ yields:

$$
\left(\begin{array}{l}
p^{\left(\mathrm{S}_{0}\right)} \\
p^{\left(\mathrm{S}_{1}\right)} \\
p^{\left(\mathrm{S}_{n}\right)} \\
p^{\left(\mathrm{T}_{1}\right)} \\
p^{\left(\mathrm{T}_{n}\right)}
\end{array}\right)=\frac{1}{C}\left(\begin{array}{c}
k_{10}^{(\mathrm{T} \rightarrow \mathrm{S})} k_{n 1}^{(\mathrm{S})} k_{n 1}^{(\mathrm{T})}\left(k_{10}^{(\mathrm{S})}+k_{11}^{(\mathrm{S} \rightarrow \mathrm{T})}\right) \\
k_{01}^{(\mathrm{S})} k_{10}^{(\mathrm{T} \rightarrow \mathrm{S})} k_{n 1}^{(\mathrm{S})} k_{n 1}^{(\mathrm{T})} \\
k_{01}^{(\mathrm{S})} k_{10}^{(\mathrm{T} \rightarrow \mathrm{S})} k_{1 n}^{(\mathrm{S})} k_{n 1}^{(\mathrm{T})} \\
k_{01}^{(\mathrm{S})} k_{11}^{(\mathrm{S} \rightarrow \mathrm{T})} k_{n 1}^{(\mathrm{S})} k_{n 1}^{(\mathrm{T})} \\
k_{01}^{(\mathrm{S})} k_{11}^{(\mathrm{S} \rightarrow \mathrm{T})} k_{n 1}^{(\mathrm{S})} k_{1 n}^{(\mathrm{T})}
\end{array}\right)
$$

$$
C=k_{10}^{(\mathrm{T} \rightarrow \mathrm{S})} k_{n 1}^{(\mathrm{S})} k_{n 1}^{(\mathrm{T})}\left(k_{10}^{(\mathrm{S})}+k_{11}^{(\mathrm{S} \rightarrow \mathrm{T})}\right)+k_{01}^{(\mathrm{S})}\left(k_{10}^{(\mathrm{T} \rightarrow \mathrm{S})} k_{n 1}^{(\mathrm{T})}\left(k_{1 n}^{(\mathrm{S})}+k_{n 1}^{(\mathrm{S})}\right)+k_{11}^{(\mathrm{S} \rightarrow \mathrm{T})} k_{n 1}^{(\mathrm{S})}\left(k_{n 1}^{(\mathrm{T})}+k_{1 n}^{(\mathrm{T})}\right)\right) .
$$

Where $C$ normalizes the sum of $p^{(\cdot)}$ to 1 . The occupation probabilities can be written in relation to $p^{\left(\mathrm{S}_{1}\right)}$ :

$$
\begin{aligned}
& p^{\left(\mathrm{S}_{n}\right)}=p^{\left(\mathrm{S}_{1}\right)} \frac{1}{k_{n 1}^{(\mathrm{S})}} k_{1 n}^{(\mathrm{S})} \\
& p^{\left(\mathrm{T}_{1}\right)}=p^{\left(\mathrm{S}_{1}\right)} \frac{k_{11}^{(\mathrm{S} \rightarrow \mathrm{T})}}{k_{10}^{(\mathrm{T} \rightarrow \mathrm{S})}} \\
& p^{\left(\mathrm{T}_{n}\right)}=p^{\left(\mathrm{S}_{1}\right)} \frac{k_{11}^{(\mathrm{S} \rightarrow \mathrm{T})}}{k_{10}^{(\mathrm{T} \rightarrow \mathrm{S})} k_{n 1}^{(\mathrm{T})}} k_{1 n}^{(\mathrm{T})}
\end{aligned}
$$

Under the experimental conditions used in this thesis, we can assume that the dye is not saturated by the excitation, i.e. the most probable state is $\mathrm{S}_{0}$. With $p^{\left(\mathrm{S}_{0}\right)} \gg p^{\left(\mathrm{S}_{i}, \mathrm{~T}_{i}\right)}, p^{\left(\mathrm{S}_{1}\right)}$ simplifies to:

$$
p^{\left(\mathrm{S}_{1}\right)} \approx \frac{k_{01}^{(\mathrm{S})}}{k_{10}^{(\mathrm{S})}+k_{11}^{(\mathrm{S} \rightarrow \mathrm{T})}}
$$

The bleaching rate is now:

$$
r_{\text {bleach }}=\frac{k_{01}^{(\mathrm{S})}}{k_{10}^{(\mathrm{S})}+k_{11}^{(\mathrm{S} \rightarrow \mathrm{T})}}\left(k_{\text {bleach }}^{\left(\mathrm{S}_{1}\right)}+k_{\text {bleach }}^{\left(\mathrm{S}_{n}\right)} \frac{k_{1 n}^{(\mathrm{S})}}{k_{n 1}^{(\mathrm{S})}}+k_{\text {bleach }}^{\left(\mathrm{T}_{1}\right)} \frac{k_{11}^{(\mathrm{S} \rightarrow \mathrm{T})}}{k_{10}^{(\mathrm{T} \rightarrow \mathrm{S})}}+k_{\text {bleach }}^{\left(\mathrm{T}_{n}\right)} \frac{k_{11}^{(\mathrm{S} \rightarrow \mathrm{T})} k_{1 n}^{(\mathrm{T})}}{k_{10}^{(\mathrm{T} \rightarrow \mathrm{S})} k_{n 1}^{(\mathrm{T})}}\right)
$$

The laser intensity dependent rate constants are: 


$$
\begin{aligned}
& k_{01}^{(\mathrm{S})}=\sigma_{01}^{\mathrm{S}, \mathrm{exc}} I_{\text {exc }}+\sigma_{01}^{\mathrm{S}, \mathrm{STED}} I_{\mathrm{STED}} \\
& k_{10}^{(\mathrm{S})}=\frac{1}{\tau_{\mathrm{S}_{1}}}+\sigma_{\text {stim }} I_{\mathrm{STED}} \\
& k_{1 n}^{(\mathrm{S})}=\sigma_{1 n}^{\mathrm{S}, \mathrm{exc}} I_{\mathrm{exc}}+\sigma_{1 n}^{\mathrm{S}, \mathrm{STED}} I_{\mathrm{STED}} \\
& k_{1 n}^{(\mathrm{T})}=\sigma_{1 n}^{\mathrm{T}, \mathrm{exc}} I_{\mathrm{exc}}+\sigma_{1 n}^{\mathrm{T}, \mathrm{STED}} I_{\mathrm{STED}}
\end{aligned}
$$

simplifying (4.18) yields:

$$
r_{\text {bleach }}=\frac{\sigma_{01}^{\mathrm{S}, \text { exc }} I_{\text {exc }}+\sigma_{01}^{\mathrm{S}, \mathrm{STED}} I_{\mathrm{STED}}}{a+\sigma_{\mathrm{stim}} I_{\mathrm{STED}}} \quad\left(b+I_{\mathrm{exc}} \delta_{1 \mathrm{n}}^{\mathrm{exc}}+I_{\mathrm{STED}} \delta_{1 \mathrm{n}}^{\mathrm{STED}}\right)
$$

where

$$
\begin{aligned}
a & =1 /\left(\tau_{\mathrm{S}_{1}}\right)+k_{11}^{(\mathrm{S} \rightarrow \mathrm{T})} \\
b & =k_{\text {bleach }}^{\left(\mathrm{S}_{1}\right)}+k_{\text {bleach }}^{\left(\mathrm{T}_{1}\right)} k_{11}^{(\mathrm{S} \rightarrow \mathrm{T})} / k_{10}^{(\mathrm{T} \rightarrow \mathrm{S})} \\
\delta_{1 n}^{\text {exc,STED }} & =k_{\text {bleach }}^{\mathrm{S}_{n}} \sigma_{1 n}^{\mathrm{S}, \text { exc, STED }} \frac{1}{k_{n 1}^{(\mathrm{S})}}+k_{\text {bleach }}^{\mathrm{T}_{n}} \sigma_{1 n}^{\mathrm{T}, \text { exc,STED }} \frac{k_{11}^{(\mathrm{S} \rightarrow \mathrm{T})}}{k_{10}^{(\mathrm{T} \rightarrow \mathrm{S})} k_{n 1}^{(\mathrm{T})}}
\end{aligned}
$$

The stimulated emission cross section $\sigma_{\text {stim }}$ can be derived from the saturation intensity (see Figure A.10) with (4.17) to

$$
\sigma_{\text {stim }}=a / I_{\text {sat }}=a /\left(0.95 \cdot 10^{24} \mathrm{~cm}^{-2} \mathrm{~s}^{-1}\right)=3.1 \cdot 10^{-16} \mathrm{~cm}^{2}
$$

$a$ was set to $2.9 \cdot 10^{8} \mathrm{~s}^{-1}$, corresponding to a fluorescence lifetime of $\approx 3.5 \mathrm{~ns}$. The intersystem crossing rate $k_{11}^{(\mathrm{S} \rightarrow \mathrm{T})}$ can be neglected in comparison to $1 / \tau_{\mathrm{S}_{1}}$. The excitation cross section for Atto647N is $\sigma_{\text {exc }}=2.5 \cdot 10^{-16} \mathrm{~cm}^{2}$ [81].

The $\delta$ factors combine the bleaching rate constants of the $S_{n}$ and $T_{n}$ states with the excitation cross section and relaxation rate constants. These parameters cannot be distinguished reliably by the experiments carried out.

\subsubsection{Analysis of the Bleaching Data}

The measurement procedure is described in detail in section 4.1.4. The phase plate was removed from the STED beam path and the foci of STED and excitation lasers were overlaid. Single molecules were located with a confocal overview image and measured individually. The measured fluorescence signal was summed up in intervals of $30 \mathrm{~ms}$ (Figure A.6c), which is the time resolution dictated by the chopper frequency (see below). The bleaching times of approximately 100 molecules were measured for each data point.

As the fluorescence is inhibited by the STED laser, the signal is too low to distinguish between bleached and unbleached states. For this reason, a chopper was used to modulate the STED beam at $30 \mathrm{~Hz}$. The part of the signal with blocked STED laser was used for calculating the bleaching time. The probability that a molecule bleaches within one measurement period $\delta t$ is then: 


$$
p_{\text {combined }}^{\text {bleach }}=p_{\text {exc }}^{\text {bleach }}+p_{\text {exc }+ \text { STED }}^{\text {bleach }}
$$

The mean survival time $\tau$ was measured. Since $\tau \sim\left(p^{\text {bleach }}\right)^{-1}$ :

$$
\tau_{\text {exc }+ \text { STED }}=\frac{\tau_{\text {exc }} \tau_{\text {combined }}}{\tau_{\text {exc }}-\tau_{\text {combined }}}
$$

where $\tau_{\text {combined }}, \tau_{\text {exc }}$ are easily measured.

Two ways of calculating the bleaching times from the raw data were evaluated:

- Taking the mean of all measured individual bleaching times, where the bleaching time was defined as data points with fluorescence signal above a threshold (see Figure A.6).

- Summing all individual fluorescence signals and fitting an exponential decay to the resulting curve (Figure A.8a).

In the first method, long dark times ( $>30 \mathrm{~ms})$ do not add to the measured bleaching time. It is unclear how these dark states contribute to photobleaching (discussed in section 5). However, it turned out that dark states of a few ms exist and might play a considerable role in bleaching. Yet, as the STED/excitation period is $33 \mathrm{~ms}$, their role cannot be reliably measured.

The second method does not account for blinking events. Also, if two molecules are measured simultaneously, both individual bleaching events are considered in the analysis.

Both methods were applied in the data analysis without considerable differences in the resulting bleaching rates (see Figure A.9). In this section, only the first method is used, as one can derive a reasonable value for the error of the measurement.

The statistical measurement error is derived assuming a normal distributed $\tau$. The standard deviation of the mean is then $\Delta\langle\tau\rangle=\sqrt{\operatorname{var}\left(\tau_{\text {single mol }}\right)} / \sqrt{N_{\text {single mol }}}$. According to error propagation:

$$
\Delta \tau_{\text {exc }+ \text { STED }}=\left(\frac{\tau_{\text {exc }}}{\tau_{\text {exc }}-\tau_{\text {combined }}}+\frac{\tau_{\text {combined }} \tau_{\text {exc }}}{\left(\tau_{\text {exc }}-\tau_{\text {combined }}\right)^{2}}\right) \Delta \tau_{\text {combined }}
$$

The bleaching time for excitation irradiation is easily measured. Values were repeatable for the whole course of measurements. Therefore, $\tau_{\text {exc }}$ was computed from the linear fit. For comparison, some of the graphs also include the measured $\tau_{\text {exc }}$, determined with above equation (4.22). The error $\delta \tau_{\text {exc }}$ is therefore of systematic nature and was not considered for the error calculation. The graphs show the bleaching rate which is $r_{\text {bleach }}=1 / \tau$. The error is $\Delta r_{\text {bleach }}=\Delta \tau / \tau^{2}$.

\subsubsection{Fitting the Model}

To fit the model of equation (4.20) to the data shown in Figure 4.7, an iterative approach was used to reduce the complexity of the system.

Bleaching by the excitation laser: First, measurements without the STED laser allow fitting $b$ and $\delta_{1 n}^{\text {exc }}$. The data is shown in Figure 4.7a. The bleaching rate scales linear with the excitation power, i.e. the excitation power is too low to cause considerable bleaching from the higher excited states. 


\subsection{Bleaching in STED Microscopy}

Bleaching by the STED laser: The STED laser is modulated by a chopper. The chopper signal is used to inhibit triggering the excitation diode, so the lasers are on alternately. The bleaching behaviour is shown in Figure 4.7b. Although considerable bleaching was observed, the rate is dependent on the chopper frequency and the excitation power, which indicates that the bleaching is not solely by the STED laser. A dependency of the bleaching rate with the chopper frequency was not observed for the measurements where both, excitation and STED laser, were on simultaneously.

The bleaching rate in these measurements is approximately four times lower than in the combined illumination measurements. However, both lasers are not switched on simultaneously for more than $5 \%$ of the measurement period. Therefore, it is speculated that the bleaching reaction occurs from a long-lived excited state of $\approx$ ms lifetime, while the bleaching purely through the STED laser can be neglected. The factor $\sigma_{01}^{\mathrm{S}, \mathrm{STED}}$ is therefore set to 0 .

Further evidence for this conclusion is given in the next paragraph.

Protective effect of stimulated emission: The factor $\sigma_{\text {stim }} I_{\text {STED }}$ in (4.20) indicates that the STED laser can reduce bleaching by lowering the lifetime of the $S_{1}$ state: A lower lifetime means less bleaching from the $S_{1}$, less population of the triplet state and a lower probability of excitation to the higher excited states. To test this hypothesis, the pulse timing between the STED and excitation lasers was offset. As can be seen in Figure $4.7 \mathrm{c}$ the bleaching rate increases strongly. As the $S_{1}$ lifetime is considerably lower than the time between excitation and depletion pulse, the bleaching is caused solely by excitation to higher triplet states.

A notable feature is that the bleaching rate scales linear with the STED power in the $20 \mathrm{~ns}$ timing offset measurement. This is a strong indication that the excitation cross section $\sigma_{01}^{\text {STED }}$ for the STED laser is low compared to $\sigma_{01}^{\mathrm{exc}}$, or there would be a quadratic component in the bleaching rate.

Combined bleaching rate of excitation and STED: With above considerations equation (4.20) reduces to:

$$
r_{\text {bleach }}=\frac{\sigma_{01}^{\text {exc }}}{a+\sigma_{\text {stim }} I_{\text {STED }}} I_{\text {exc }}\left(b+I_{\mathrm{STED}} \delta_{1 n}^{\mathrm{STED}}\right)
$$

with $\delta_{1 n}^{\text {STED }}$ as the only unknown factor. This equation has one big flaw: The bleaching rate saturates for STED powers of a few milliwatt. Moreover, as the protective STED effect indeed reduces the bleaching rate, above equation would predict a lower bleaching rate for all STED powers.

The bleaching rate usually shows a plateau or slight decrease for STED powers $<1 \mathrm{~mW}$, followed by a roughly linear increase (Figure $4.7 \mathrm{~d}$ ). Notably, the plateau is also apparent in the measurement series with the 8 ns delayed STED pulse. This is unexpected, as no fluorescence depletion was observed ${ }^{4}$ (see Figure A.10). The data shown in the graphs are independent measurement series, where the STED pulse timing of Series 1 and 2 is set to the optimum for time-gated detection, i.e. the STED pulse is slightly delayed. For Series 3, the STED pulse was directly overlaid with the excitation pulse, leading to an apparently higher saturation intensity (see appendix). Evidently, the experimental data for the bleaching rate

\footnotetext{
${ }^{4}$ However, the detection was time gated and residual fluorescence out of the time frame could still be affected by the STED pulse
} 


\section{Scanning Field of Views Below the Diffraction Limit}

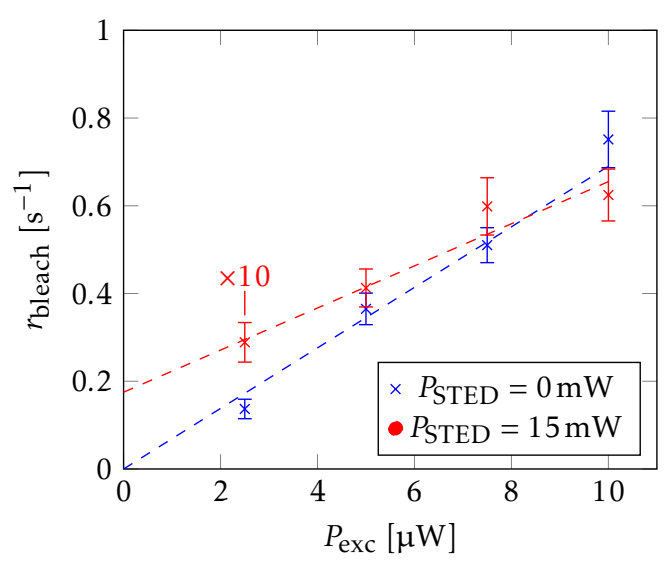

(a) Bleaching rate depending on excitation power

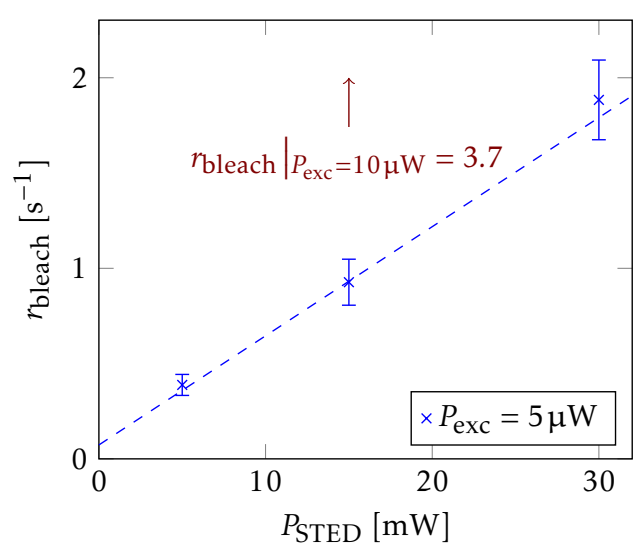

(b) Bleaching with STED laser only

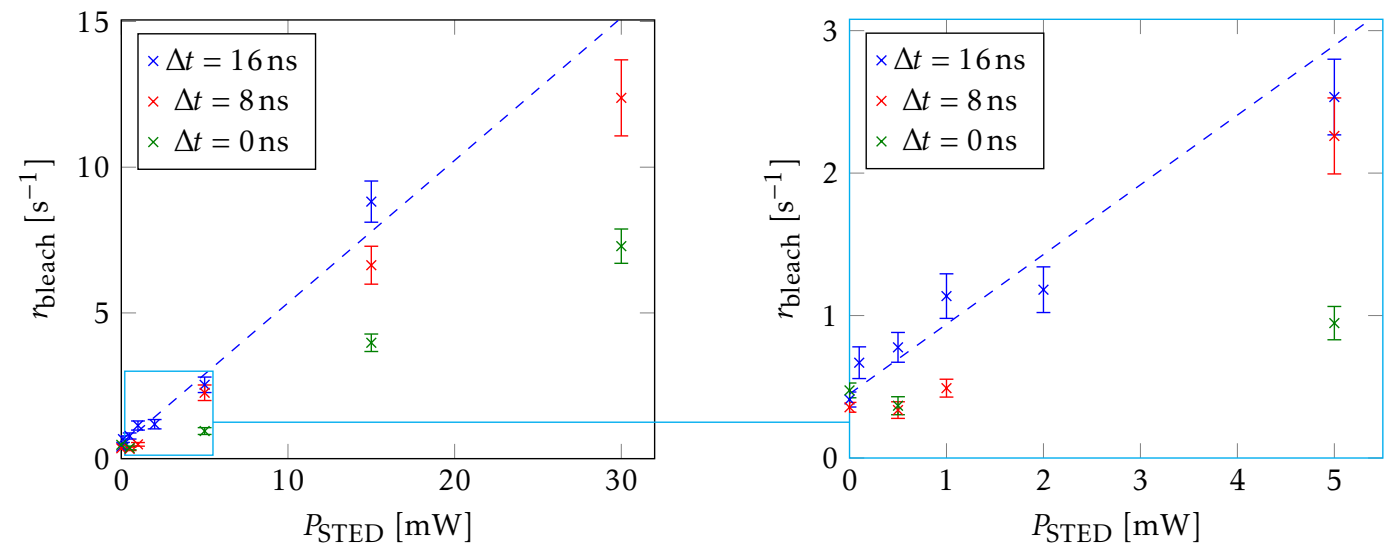

(c) Bleaching rate for different time delays between STED and excitation.
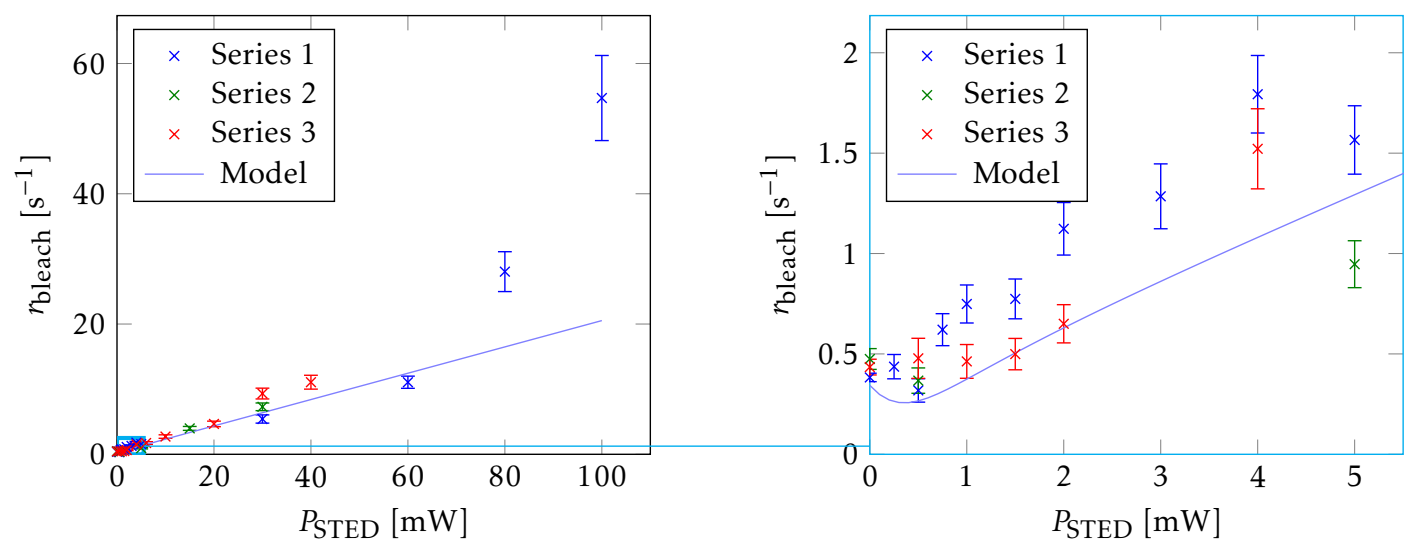

(d) Bleaching rate of STED and exitation laser combined

Figure 4.7. Single molecule bleaching data. Each data point is the mean bleaching time of $\approx 100$ Atto647N molecules. To identify the bleaching pathway, the laser powers and laser pulse timing were varied. 


\begin{tabular}{|lrrl|}
\hline$\sigma_{01}^{\text {S, exc }}$ & & $2.5 \cdot 10^{-16}$ & $\mathrm{~cm}^{2}$ \\
$\sigma_{01}^{\text {S, STED }}$ & $*$ & 0 & $\mathrm{~cm}^{2}$ \\
$\delta_{1 n}^{\text {exc }}$ & $*$ & 0 & $\mathrm{~cm}^{2}$ \\
$\delta_{1 n}^{\text {STED }}$ & $*$ & $1 \cdot 10^{-23}$ & $\mathrm{~cm}^{2}$ \\
$\mathrm{a}$ & & $2.9 \cdot 10^{8}$ & $\mathrm{~s}^{-1}$ \\
$\mathrm{~b}$ & $*$ & 42 & $\mathrm{~s}^{-1}$ \\
$\sigma_{\text {stim }}$ & $*$ & $3.1 \cdot 10^{-16}$ & $\mathrm{~cm}^{2}$ \\
$\eta$ & $*$ & $3.1 \cdot 10^{-25}$ & $\mathrm{~cm}^{2} \mathrm{~s}$ \\
\hline
\end{tabular}

Table 4.4. Parameters of the bleaching model, referring to equations (4.20) and (4.24). Values marked with * were derived from the measurements. Please note that the value for the stimulated emission cross section was determined assuming CW STED and is not corrected for the pulse repetition rate.

is not accurate enough to draw conclusions on the detailed bleaching behavior. Yet three main features can be identified: For low STED powers there is a slight decrease or at least a plateau in the bleaching rate, followed by a roughly linear increase.

To adapt the model to the data, a saturation factor for the protective effect of the stimulated emission was introduced. A reason for the saturation might be a fast intersystem crossing to the triplet state directly after the initial excitation into high vibrational states [82], or after excitation to $S_{n}$ by the STED laser. As the photophysical mechanisms involved in bleaching are complex and the data is noisy, a saturation factor of the simple form $1 /\left(1+\left(\eta * I_{\mathrm{STED}}\right)\right)$ is added to account for the simulated emission at low powers and the saturation at high powers. The bleaching rate is now:

$$
r_{\text {bleach }}=\frac{\sigma_{01}^{\text {exc }}}{a+\sigma_{\text {stim }} I_{\mathrm{STED}} \frac{1}{1+\left(\eta * I_{\mathrm{STED}}\right)}} I_{\mathrm{exc}}\left(b+I_{\mathrm{STED}} \delta_{1 n}^{\mathrm{STED}}\right)
$$

This model was fitted to the data of the measurement Series 3 (fit parameters: $\eta, \delta_{1 n}^{\mathrm{STED}}$, results in Table 4.4). Please note that errors were omitted on purpose for the results in Table 4.4. While the fit indicated errors below $10 \%$ for $b$ and $\sigma_{\text {stim }}$ (which is, in my experience, too low), they were one order of magnitude higher as the fitted values for $\eta$ and $\delta_{1 n}^{\text {STED }}$. The reason for this large error is (besides the noisy data) that the slope of the bleaching rate at high STED powers is $\sim \delta_{1 n}^{\mathrm{STED}} * \eta$ and precise fitting is not possible. These values should therefore be used with caution.

Summary: The bleaching behavior of Atto647N was modeled using a steady-state approach on a 5-level system. It could be shown that excitation from the triplet or similar long-lived states is the main bleaching pathway induced by the STED laser. Stimulated emission lowers the lifetime of the $S_{1}$ excited state, thus indirectly reducing the triplet population. Therefore, the STED laser can reduce the bleaching rate, which was observed for STED intensities $\approx I_{\text {sat }}$. While the derived model can be applied well to the bleaching by the excitation and STED laser separately, it fails in the case of simultaneous excitation and stim- 


\section{Scanning Field of Views Below the Diffraction Limit}

ulated emission. A factor introducing a saturation of the protective STED effect was added to the model to roughly match the bleaching data. The model would now predict a linear increase in the bleaching rate with the STED laser power, with a lowered slope or reduced bleaching at low powers. This approach roughly describes the shape of the experimental bleaching curve, yet cannot deliver a reliable model.

A main factor for the poor results is the strong noise in the bleaching measurements: Several measurement series have been acquired, exhibiting qualitatively similar and repeatable behavior. Individual measurement data points should have a statistical error of $10 \%$ (for a measurement with 100 molecules), yet scatter stronger than expected. Also, deviations from the exponential bleaching distribution were sometimes observed. No apparent cause for this behavior could be determined, more data on these issues is presented in the appendix.

In conclusion, equation (4.24) provides a qualitative description of the bleaching behavior, exhibiting reduced bleaching for low STED powers and a linear increase at higher powers. Although the sample was chosen to provide long-term stability ${ }^{5}$ and repeatable properties, short term variations of the measurement results render a quantitative analysis difficult.

\subsubsection{Application to STED Measurements}

Although the bleaching model does not explain the experimental data satisfactory, it still provides a reasonably good approximation. The results can be used to calculate the bleaching behavior for relevant experimental conditions in STED microscopy. A measurement usually consist of a two-dimensional scan of a certain area $A$ where the dwell time $t_{\mathrm{d}}$ and pixel size $\delta x$ determine the scanning speed. The probability that a dye in the center of the scanning area bleaches during one frame is

$$
\begin{aligned}
p_{\text {bleach }}^{\text {frame }} & =1-\prod_{\text {pixels }}\left(1-p_{\text {bleach }}(\text { pixel })\right) \\
& =1-\prod_{\text {pixels }}\left(\exp \left(-r_{\text {bleach }}(\text { pixel }) * t_{\mathrm{d}}\right)\right)
\end{aligned}
$$

$r_{\text {bleach }}$ (pixel) is the bleaching rate of equation (4.18).

In Figure 4.9 the bleaching simulation is compared to the experimental results. The experiments are carried out on the same single-molecule sample as in the last section. Each graph shows the mean frames for the molecules that can be acquired before photobleaching, averaged over 50-100 molecules. To avoid drift-induced measurement artifacts, the STED and confocal measurements were performed alternately, while also altering the scan size.

For the simulation, the STED intensity distribution was measured with gold beads and fitted with the function:

$$
I(r)=I_{0} r^{2} \exp \left(-\left(r / r_{0}\right)^{2}\right)
$$

resulting in $r_{0}=0.234 \mu \mathrm{m}$, corresponding to an intensity peak-to-peak distance of $470 \mathrm{~nm}$, $I_{0}$ was calculated according to the resolution (experimentally determined FWHM: $40 \mathrm{~nm}$ at $P_{\text {STED }}=90 \mathrm{~mW}$ and $80 \mathrm{~nm}$ at $P_{\text {STED }}=20 \mathrm{~mW}$ ). The excitation focus was approximated by a Gaussian function with an integral power flux of $5 \mu \mathrm{W}$ and a FWHM of $250 \mathrm{~nm}$.

\footnotetext{
${ }^{5}$ No significant deviations in the behavior were observed over several weeks
} 

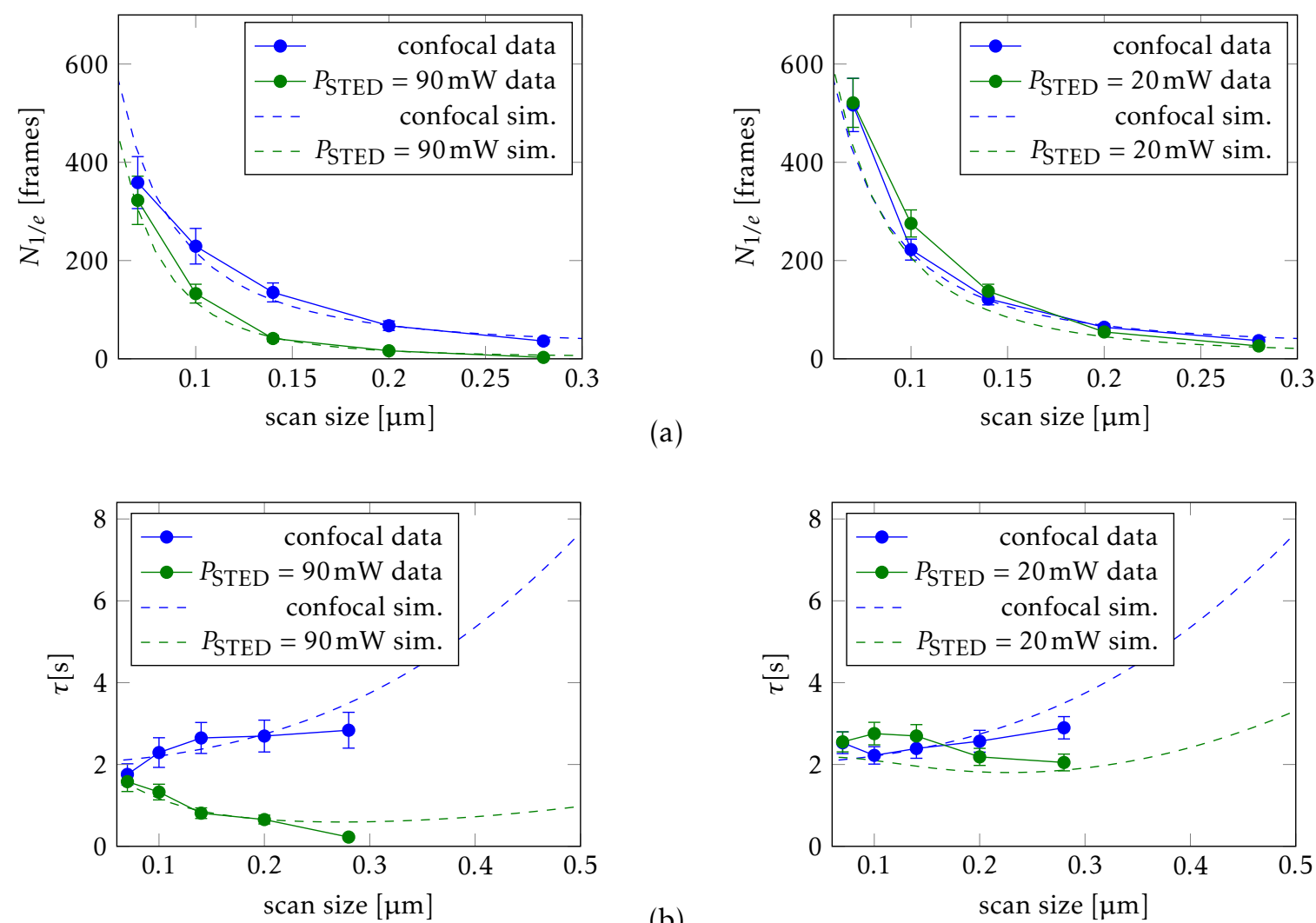

(a)

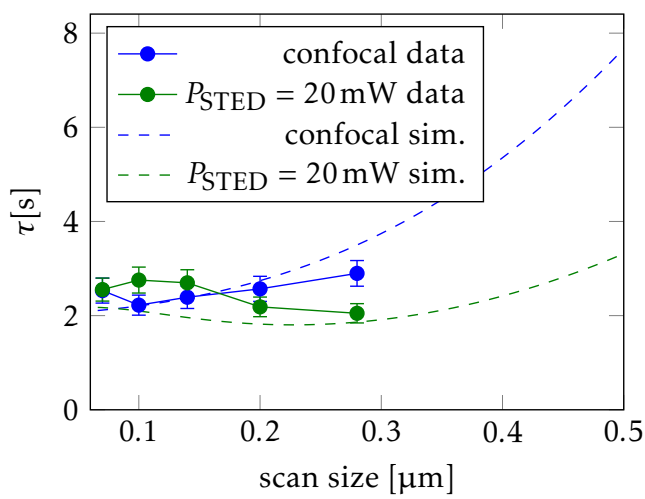

Figure 4.8. Experimental (points) and simulated (dashed lines) data for the mean number of measured frames $N(\mathrm{a})$, and total measurement time (b), until a single Atto647N molecule bleaches. The parameters are: $10 \mathrm{~nm}$ pixel size, $100 \mu$ s dwell time, $5 \mu \mathrm{W}$ excitation power. The scan area is a square with the according edge length.

Figure $4.8 \mathrm{a}$ and $4.8 \mathrm{~b}$ compare the experimental results to the simulation for two different STED powers. The simulation is in good agreement with the experiments, giving quantitative results within the limitations of the experiment. Figure 4.9a depicts the relative increase in the number of frames $N$ that can be acquired when reducing the scan size from $200 \times 200 \mathrm{~nm}^{2}$ to $100 \times 100 \mathrm{~nm}^{2}$, depending on the resolution: In the confocal case the improvement lies at 3.3, which is consistent with the expectation that it should be lower than 4 , as the excitation FWHM is $250 \mathrm{~nm}$ and the measurement time of the smaller region is $1 / 4$ of the larger one. The relative improvement rises up to 10 for $10 \mathrm{~nm}$ resolution. The experiments with single molecules and DNA origami confirm the expected behavior. However, this model does not fit the data acquired on Star Red labeled nanobodies (Section 4.3.2). Further experiments will have to determine the bleaching parameters of Star Red to get a better estimate.

Figure $4.9 \mathrm{~b}$ shows the increase in $N$ for various scan sizes relative to big scan areas. As can be seen, the improvement easily exceeds a factor of 100 at $20 \mathrm{~nm}$ resolution and scan sizes below $70 \times 70 \mathrm{~nm}^{2}$. It is shown in Figure $4.10 \mathrm{~b}$ of the next section on labeled DNA origami that these parameters are not impractical, i.e. sufficient structural information on the sample is obtained. It is, however, experimentally challenging to verify the improvement of $N$, as 
almost no fluorescence can be obtained in the big area scans.

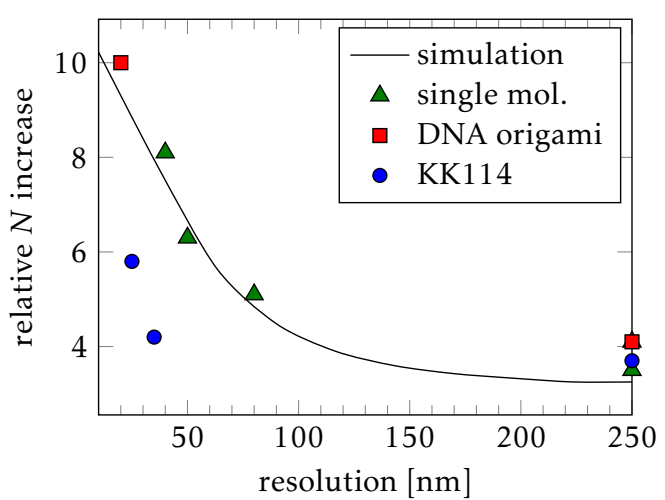

(a) $(100 \mathrm{~nm})^{2} /(200 \mathrm{~nm})^{2}$

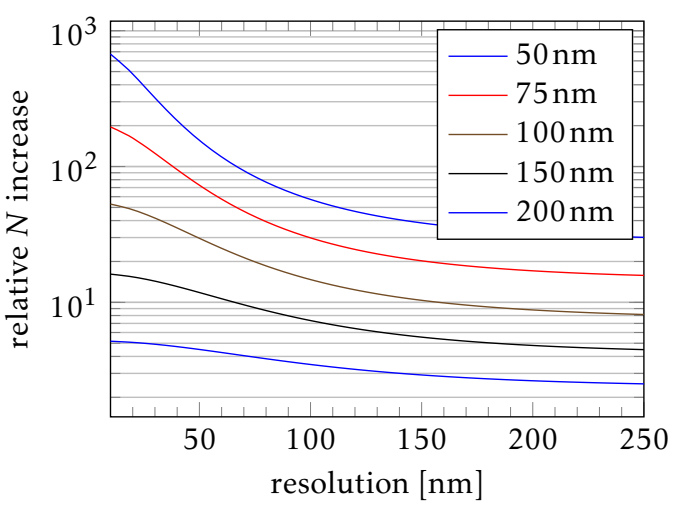

(b) small scan field / big scan field

Figure 4.9. (a) shows the relative increase in $N$ between a $200 \mathrm{~nm}$ and a $100 \mathrm{~nm}$ scan, depending on the resolution. (b) shows the relative increase in $N$ of various scan sizes and resolution compared to a big scan.

\subsection{Application Examples}

\subsubsection{DNA Origami}

Moving from imaging of single molecules to more complex structures, labeled DNA origami nanorulers provide a well defined test sample. A problem when comparing the imaging performance of microscopes is the varying sample quality. Especially in super-resolution microscopy, where only few fluorophores contribute to the signal of each data point, differences in dye stability, fluorescent background, labeling density etc. have a strong impact on the quality of the resulting image. DNA nanorulers aim to provide a well defined, easily available calibration and test sample. DNA can be synthesized to self-assemble into predefined nanostructures [83]. Using labeled staple strands one can control the dye position accurately and produce a defined dye pattern [84]. The sample used (STED 27R, GATTAquant, Braunschweig, Germany) features two parallel rows of up to 12 Atto647N molecules each, separated by $27 \mathrm{~nm}$. Imaging the dye rows separately with a large field of view failed, as the attainable signal was too low.

Reducing the scan field increased the acquirable signal considerably (Figure 4.10). A STED and excitation laser power of $400 \mathrm{~mW}$ and $3 \mu \mathrm{W}$ respectively was used in the measurements, as measured in the objective's back aperture. The images have a pixel size of $5 \mathrm{~nm}$ and the pixel dwell time was set to $100 \mu \mathrm{s}$. As the individual origamis are distributed with a density of $\approx 1 \mu \mathrm{m}^{-2}$, they are easily localized by a confocal overview scan. Small areas with an edge length from 50-200 $\mathrm{nm}$ were measured with and without the STED laser. All fluorescence traces of a particular scan size were added and fitted with an exponential decay, computing $N_{1 / 2}$, the number of frames after which half the fluorescence is bleached. $N_{1 / 2}$ scales with the scan area for the confocal measurements, i.e. the bleaching rate is constant in time: Half the fluorescence is bleached after $\approx 35$ frames of $200 \times 200 \mathrm{~nm}^{2}$ size. This number increases to $\approx 560$ frames for $50 \times 50 \mathrm{~nm}^{2}$ sized scan fields. The increase in fluorescence signal by a factor of 16 corresponds to the ratio of the areas. 
The bleaching reduction for the STED images is far greater than in the confocal case: $N_{1 / 2}$ increases by a factor of roughly 100 , from 1.7 to 168 , when reducing the scan size from $200 \mathrm{~nm}$ to $50 \mathrm{~nm}$. As a measure for the STED induced bleaching, the ratio between STED and confocal bleaching rate is convenient: It reduces from $\approx 20$ to $\approx 3.4$ when reducing the scan size, as the STED intensities irradiating the field of view are considerably lowered.

As only $\approx 2$ frames could be acquired for the $200 \mathrm{~nm}$ sized scan fields, larger scan areas did not result in sufficient signal: The simulation in Figure $4.9 \mathrm{~b}$ calculates a 5-fold bleaching reduction of the $200 \mathrm{~nm}$ scan field compared to a normal scan. The bleaching model derived from the single molecule measurements was applied to the data in Figure 4.10d, with a resolution of $20 \mathrm{~nm}$ and scaled by a constant factor to account for differing bleaching times due to the different mounting medium. The simulation fits the data very well.

Although the parallel rows of dye in the DNA origami can be separated in the $200 \mathrm{~nm}$ scans

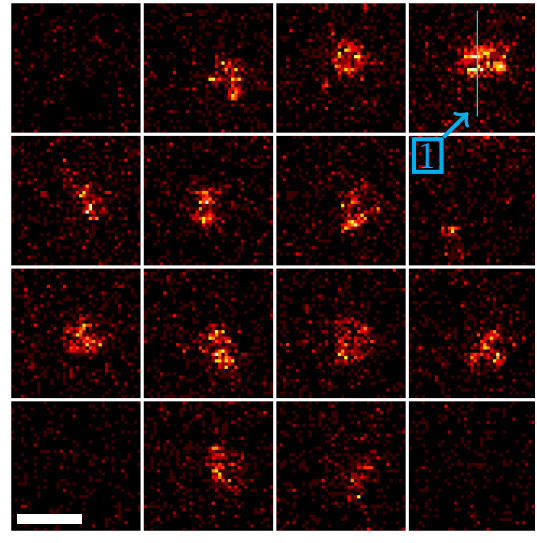

(a)

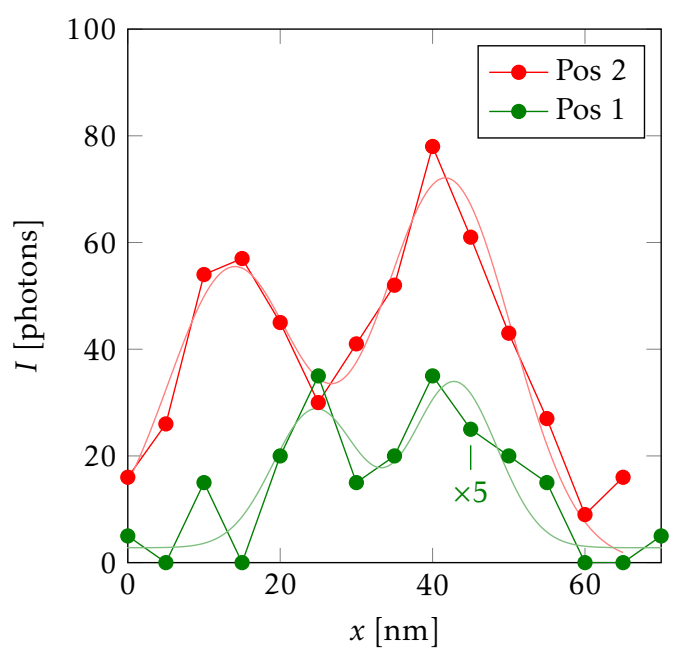

(c)

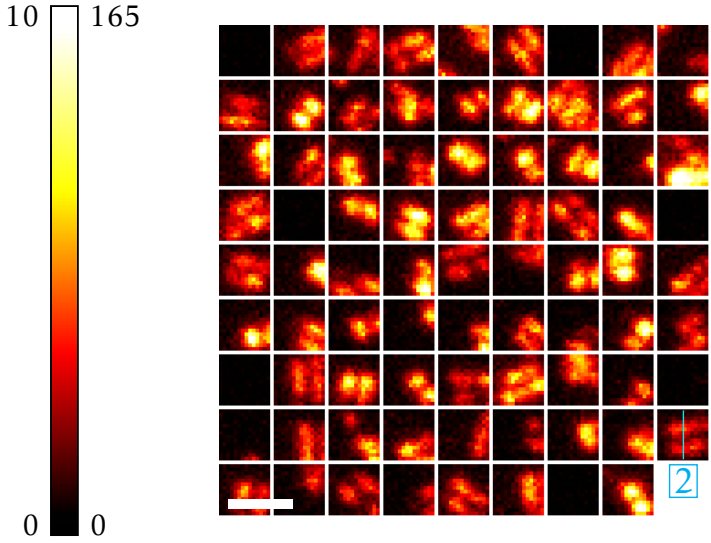

(b)

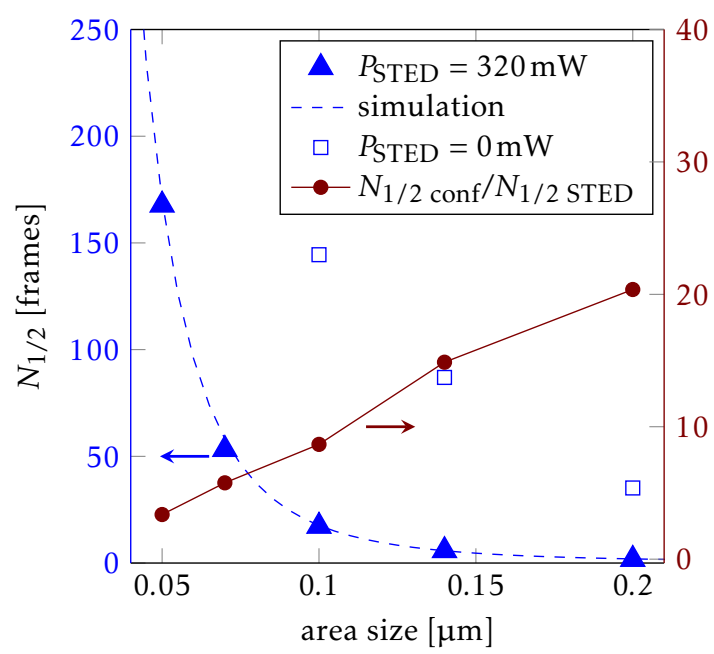

(d)

Figure 4.10. DNA origami imaged with (a) $200 \times 200 \mathrm{~nm}^{2}$ and (b) $70 \times 70 \mathrm{~nm}^{2}$ sized scan fields. The number of frames added [(a): 2; (b): 70] correspond to the respective $N_{1 / 2}$. (c): Line profile and fit at the indicated positions (scaled by a factor of 5 for the $200 \mathrm{~nm}$ scan). (d): Bleaching times $N_{1 / 2}$ (blue) and ratio of STED to confocal bleaching rate (red). Scale bar: $100 \mathrm{~nm}$. 
well enough (Figure 4.10a), the signal-to-noise ratio is unsatisfying. In most cases the structure of the sample remains unclear. Using larger scan fields yields even less signal. Figure $4.10 \mathrm{~b}$ shows images with $70 \mathrm{~nm}$ sized scan fields, which increases the available signal approximately 30 -fold. Please note that due to the relatively large shot noise at low signal, the absolute peak intensities scale differently: At a mean intensity of 7 counts, more than $10 \%$ of the data points will have an intensity $>10$ counts (or higher than $50 \%$ of the mean value). At 200 counts, less than $10 \%$ of the data points will have a value $>10 \%$ of the mean. The line profiles were fitted with two Gaussian peaks of the same FWHM, producing a FWHM of $13 \mathrm{~nm}$ with $18 \mathrm{~nm}$ peak separation for the low-signal $200 \mathrm{~nm}$ image, and a FWHM of $19 \mathrm{~nm}$ with $28 \mathrm{~nm}$ peak separation for the $70 \mathrm{~nm}$ scan (fit errors $\approx 2 \mathrm{~nm}$ for all values). Due to the high sample heterogeneity and low signal of the $200 \mathrm{~nm}$ images, a detailed statistical analysis by fitting many origami structures was not carried out. However, there is no reason for the resolution to be higher in the larger scan fields than in the small images. This example rather stresses the importance of a high signal when investigating small structures quantitatively. More details on the origami data can be found in the appendix Section A.2. 


\subsubsection{Nanobodies}

One of the most successful labeling techniques for imaging in biology is the immunolabeling with antibodies. Antibodies can be tagged with a marker and specifically bind to a target structure. In fluorescence microscopy, fluorophores coupled to the antibodies provide the signal which is used to uncover the target structure. As the number of dye molecules attached to an antibody is usually limited to $\approx 5$ [85], the signal is often boosted with labeled secondary antibodies, targeting the unlabeled primary antibody. Several secondary antibodies can bind to one primary antibody. Thus, the number of dye molecules at the target is increased. As these secondary antibodies usually target all antibodies originating from a specific organism (e.g. mouse), they can be reused for different primary antibodies. This makes indirect antibody staining a very flexible and efficient method with high signal yield and contrast.

The main drawback of this method in super-resolution microscopy is the size of the antibodies. The detected signal originates from the attached dye molecules, not the structure of interest, so the size of the antibodies limits the localization accuracy. Furthermore, the target protein might be in a crowded environment and inaccessible for the antibody. With typical sizes of $10 \mathrm{~nm}$ per antibody, indirect staining may shift the signal by up to $20 \mathrm{~nm}$ or result in a cluster $40 \mathrm{~nm}$ in size, thus introducing labeling artifacts [86].

A recent development is the use of single-domain antibodies, commonly called nanobodies. The only mammals producing these antibodies with no light chains and $\mathrm{CH} 1$ domain are Camelids. The antigen binding domain, called $\mathrm{V}_{\mathrm{H}} \mathrm{H}$, can be isolated. These $\mathrm{V}_{\mathrm{H}} \mathrm{H}$ fragments can be very specific and have other advantages. Most important, in the context of superresolution microscopy, is their reduced size compared to conventional antibodies. They are only around $10 \%$ as large and therefore permit a precise localization of proteins with superresolution techniques $[87,88,67]$.

Labeled nanobodies, however, have the drawback of being rather dim, due to the low degree of labeling (up to 3 dye molecules per nanobody). It is essential to retrieve as much signal as possible before the dyes bleach and their position is irretrievable.

Conventional STED microscopy fails to acquire STED images with the necessary signal-tonoise ratio to resolve the finer details of complex structures labeled with nanobodies. Reducing the scan area vastly improves the signal yield as shown in Figure 4.11. Keeping other imaging parameters constant, the signal yield increases with reduced scan range. An improvement of as high as 40 to 90 -fold for a STED power of $80 \mathrm{~mW}$ and $160 \mathrm{~mW}$ respectively was feasible, without loosing structural information by clipping the pores. The bleaching at higher STED powers was too strong for large scan fields.

The data shown in Figure 4.11 was acquired with an excitation power of $1 \mu \mathrm{W}$, a pixel size of $10 \mathrm{~nm}$ and $100 \mu \mathrm{s}$ pixel dwell time. Multiple frames of the same region were acquired and the signal of the central $10 \times 10$ pixels was summed up for each frame. The intensity curve of the individual pores is summed and fitted with an exponential decay and the calculated halflife time is plotted in Figure 4.11b. The error bars of the fit are much smaller than the error originating from the measurement, such as defocus and sample heterogeneity. Also, please note that the bleaching curve of the confocal scan is distorted by the fact that the investigated sample is not point-like: The signal measured originates from a diffraction-limited area which is exposed to various excitation powers. When the scan field is reduced below the size of the focus, signal from outside the scan area will have a significant contribution to the total signal measured. 

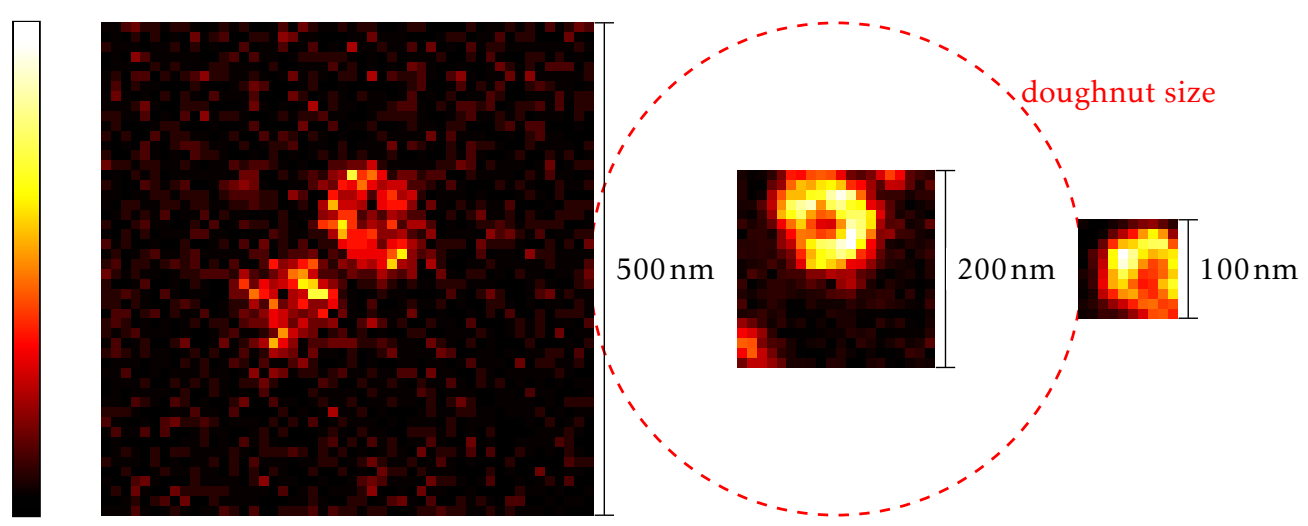

(a)

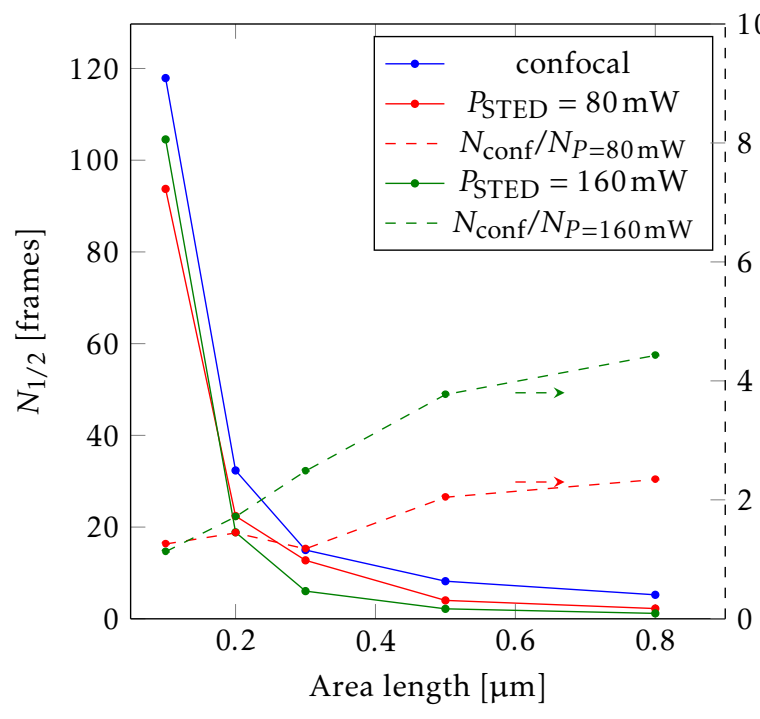

(b)

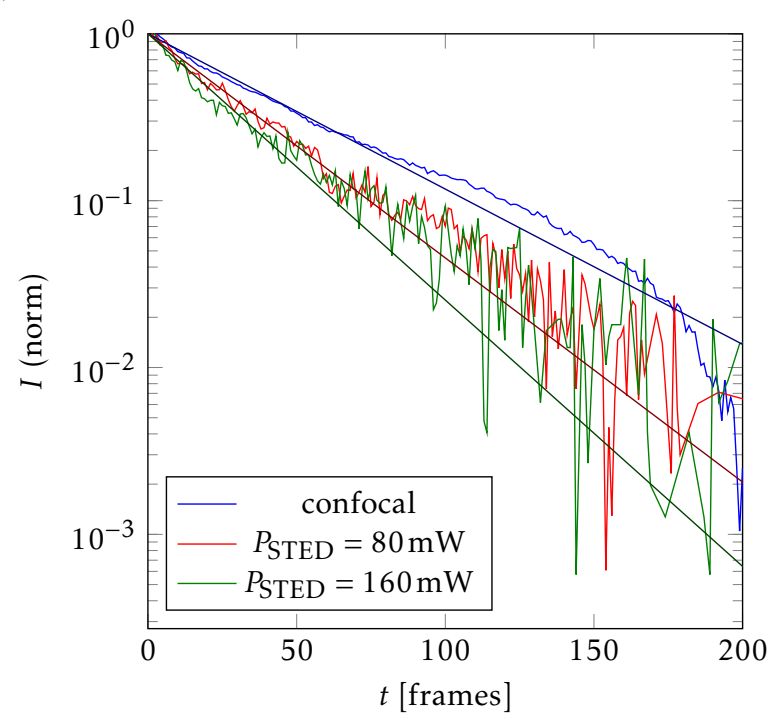

(c)

Figure 4.11. The reduction of the scan size reduces the bleaching per acquired frame. More signal can be acquired. (a) exemplifies this effect on the nuclear pore protein Nup98 labeled with nanobodies. The images show the complete field of view of 500, 200 and $100 \mathrm{~nm}$ length respectively for for STED measurements at $160 \mathrm{~mW}$ STED laser power. The signal was summed over the first $N_{1 / 2}$ frames $(2,19,104)$. The lower and upper boundaries of the color scale are $0 / 20,12 / 183$ and $175 / 972$. (b) shows $N_{1 / 2}$ for varying square scan areas. $N_{1 / 2}$ is the number of frames that has been acquired until half the fluorescence signal is bleached. The dashed lines indicate the ratio between $N_{1 / 2}$ of the confocal and STED scans. (c) shows the normalized bleaching curves and exponential fit of the sample for a scan size of $200 \mathrm{~nm}$ 
As expected, the bleaching rate for the STED measurements is several times larger compared to the confocal measurements $(\approx 5 \times$ for $160 \mathrm{~mW}$ STED power). This difference decreases significantly for reduced scan fields: When scanning an area of size $100 \times 100 \mathrm{~nm}^{2}$, the bleaching rate with STED is basically the same as in the confocal mode, while still obtaining structural details from the image.

It is clear also from the large-area STED picture in Figure 4.11a that Nup98 arranges circularly around the pore's center, yet a higher resolution is not feasible due to the strong bleaching. Scanning small areas is especially beneficial for high STED powers, as the relative reduction in bleaching is high and the resolution allows detailed imaging of the structure. Figure 4.12 demonstrates this for NUP93 and NUP98 with $100 \times 100 \mathrm{~nm}^{2}$ sized scan fields. The STED power was increased to $320 \mathrm{~mW}$ at the objective's back aperture. The pixel size is $6 \mathrm{~nm}$, the images show the sum of 17 individual measurements adding to a total dwell time of $1.7 \mathrm{~ms}$ per pixel. Evidently more substructures can be distinguished easily. Line profiles through representative structures yield a resolution of approximately $17 \mathrm{~nm}$. Like gp210 (Figure 3.7), Nup98 and Nup93 are expected to follow the 8-fold symmetry typical for the nuclear pore. While the angular distribution of the individual Nup93 spots strongly indicates towards the 8 -fold symmetry, no full rings were observed. Two reasons are identified: Although the ring's diameter is smaller than the scan field, centering individual pores precisely is difficult, as they usually can't be separated in the confocal overview. A second obstacle is the labeling density: Many pores are not completely labeled, which may be due

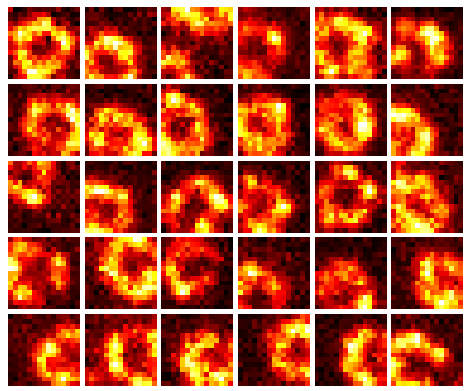

(a)

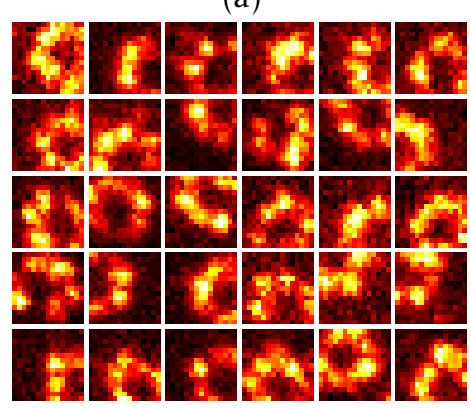

(b)

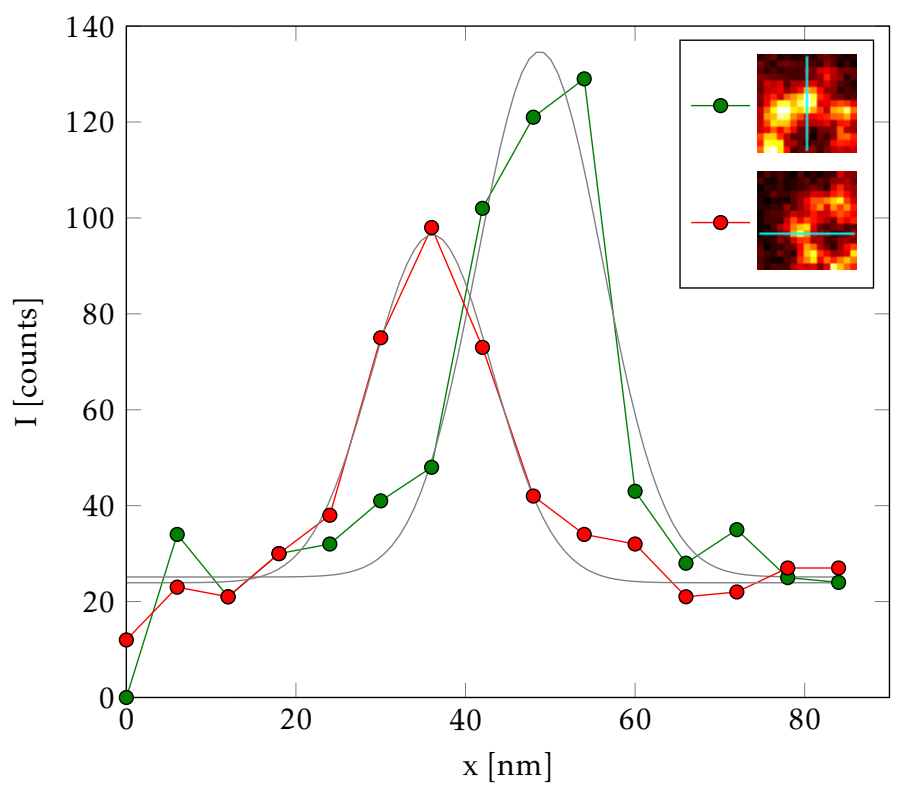

(c)

Figure 4.12. Images of nanobody-labeled Nup98 (a) and Nup93 (b). Each square has a dimension of $100 \mathrm{~nm} \times 100 \mathrm{~nm}$, the intensities were normalized to minimum and maximum values for better visibility.

(c): Line profiles of two representative positions. The data is fitted with a gaussian curve (gray). The FWHM of the fit is $17 \pm 2 \mathrm{~nm}$ (green) and $17 \pm 1.4 \mathrm{~nm}$ (red) 


\section{Scanning Field of Views Below the Diffraction Limit}

to accessibility problems, fixation artifacts or unlabeled nanobodies. While many pores are centered in the scan field, they show gaps in their circular arrangement. Furthermore, as their diameter is $60-70 \mathrm{~nm}$, the protein separation is on average $\approx 25 \mathrm{~nm}$, which is well within the resolving power of the setup. However, the distance might often be significantly lower, as the nuclear pore is not a perfectly symmetric circular structure.

As can be seen, the Nup98 images seem to be significantly more blurred, i.e. the substructures are not as apparent as for the Nup93 labeling. Whether this is due to a denser labeling or a different protein organization pattern remains to be investigated.

In the previous section, a protective effect of STED on the dye was observed on Atto647N. As the lifetime of the $S_{1}$ electronic state is reduced by the STED laser, less bleaching occurs from the $S_{1}$ state and, more importantly, the triplet state is less populated, resulting in lower bleaching. This mechanism should apply to other dyes as well, provided that the additional bleaching induced by the STED laser is low. It was also observed on Star Red, as illustrated by Figure 4.13 . The bleaching rate decreases by approximately $10 \%$ when imaging with $8 \mathrm{~mW}$ STED power. It should be noted that, as the protective effect is due to inhibiting the $S_{1}$ state, the total fluorescence acquired is still lower than in the confocal case.

The images in Figure 4.13 were taken at an excitation power of $1.5 \mu \mathrm{W}$, with a pixel size of $50 \mathrm{~nm}$ and $100 \mu \mathrm{s}$ dwell time. 1000 frames were acquired, the fluorescence time trace of the central $1 \mu^{2}$ was summed over all measurements and fitted with an exponential decay. The error of the measurement is mainly caused by defocus and sample heterogeneity (see Figure A.14) .

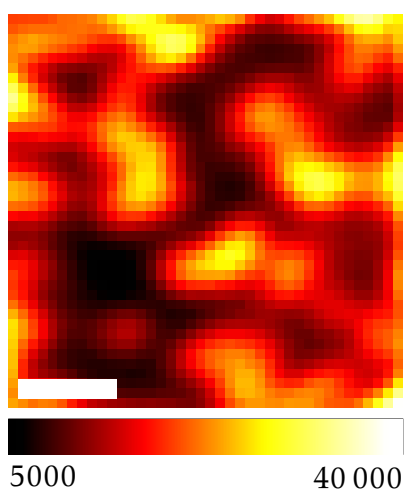

(a)

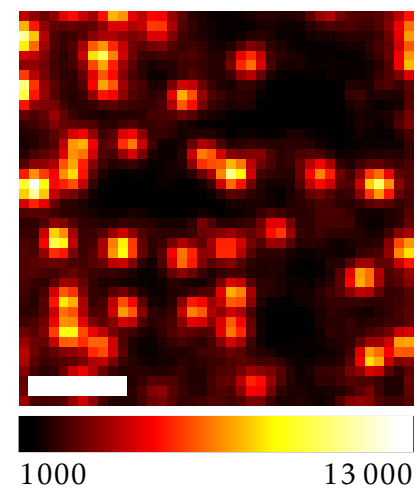

(b)

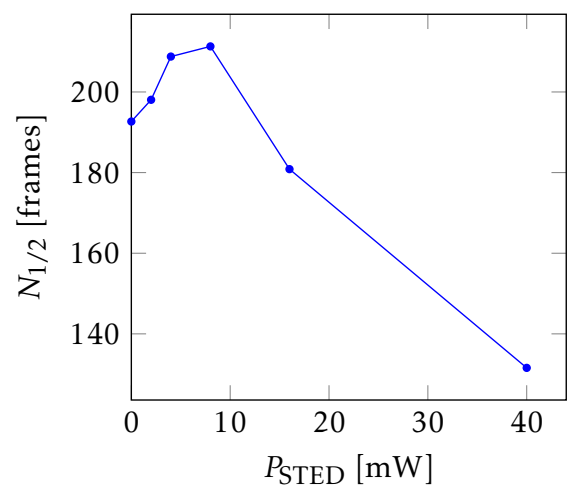

(c)

Figure 4.13. Stimulated emission can reduce the bleaching rate of Star Red, exemplified on a nanobody-labeled Nup98 sample. Confocal (a) and STED (b) image stacks of 1000 frames were acquired. The fluorescence decay was fitted with an exponential, the half-times determined for various STED powers are plotted in (c). The lowest bleaching rate is reached for $P_{\text {STED }}=8 \mathrm{~mW}$, which yields a resolution of $\approx 100 \mathrm{~nm}$. Scale bar: $500 \mathrm{~nm}$ 


\section{Discussion}

\section{Photobleaching}

Photobleaching is an irreversible reaction of the fluorophore, destroying its fluorescence ability. As photobleaching is limiting the available signal in all fluorescence applications, many studies have been conducted to investigate its mechanisms. However, the detailed reaction pathways still remain unclear, owing to the complex molecular and electronic structure of the molecules and their interaction with the environment. It is therefore not surprising that bleaching studies for one kind of dye have little significance for other dyes. Even the bleaching kinetics of the same dye vary strongly with the experimental conditions.

One of the most widely used fluorescent dyes is rhodamine 6G. ${ }^{1}$ Its use as laser dye has made it the subject of many studies investigating its photophysics at various conditions. However, already the assumption that photobleaching would follow an exponential distribution breaks down for some conditions, e.g. for rhodamine $6 \mathrm{G}$ embedded in polyvinyl alcohol [79]. In this report, Zondervan at al. demonstrated a strong deviation from an exponential decay in the bleaching kinetics of ensemble and single molecule samples. The shape of the bleaching curve was also strongly dependent on the excitation power, and whether oxygen was available in the buffer. Interestingly, photobleaching was stronger in an inert atmosphere compared to air for the ensemble measurements, while the opposite was observed in the single molecule experiments. Zondervan et al. explained this discrepancy with different subpopulations of dyes observed in the two modes.

I would like to take this study as an example to point out how sensitive bleaching measurements are even to changes in the measurement method. My goal was to precisely measure the bleaching rates for defined excitation and STED intensities. When performing ensemble experiments, it would be necessary to ensure homogeneous illumination of the sample with relevant intensities for STED microscopy. Furthermore, dye ensembles may not behave consistently: It has been shown, and repeatedly observed personally, that fluorescent dyes can effectively quench themselves in dense samples [89, 90]. Dye-dye bleaching may also be a relevant bleaching pathway to consider [91]. These factors make it challenging to produce a reliable sample that allows an accurate control of the irradiating laser power. A single molecule approach to measure the bleaching rate is therefore preferred.

Similar studies to the above (rhodamine 6G in polymethyl acrylate under vacuum [92]; in water solution $[93,94]$ ) observed only slight deviations from the exponential bleaching behavior, yet agree on the strong dependence of the effective bleaching rate on the excitation power. All three reports derived a similar bleaching model to explain their results, based on a steady state approximation of a rate model considering the ground state $S_{0}$, the excited state $S_{1}$, the triplet state $T_{1}$ and higher excited states $S_{n}, T_{n}$ of the dye. When the excitation

${ }^{1} 1128$ search results on webofknowledge.com for scientific literature including 'Rhodamine 6G' in the title as of 01.10 .2015 


\section{Discussion}

power is low, bleaching occurs from the $S_{1}$ and $T_{1}$ states and the total fluorescence measured will not depend on the excitation power.

At high excitation power, photobleaching caused by exciting electrons from the $\mathrm{T}_{1}$, or a meta-stable dark state, into higher states and a subsequent bleaching reaction dominate the bleaching process. This is indicated by a nonlinear increase in the bleaching rate with the excitation power, and is commonly referred to as two-step photolysis, as it involves two absorption events. Strong bleaching from higher states seems to be a common feature of many dyes and appears under various experimental conditions [95, 96, 97, 98]. The effect increases with the lifetime of the triplet state. Thus, removing oxygen from the sample medium may increase or decrease the bleaching rate, depending on other parameters [79, 92, 95, 91]: Free oxygen is commonly considered to be one of the main reaction partners in photobleaching, yet it is equally agreed to be a good triplet quencher. It is therefore essential, when measuring the bleaching kinetics, to ensure a stable and well controlled environment. For my experiments I used PBS as the mounting medium, providing a stable $\mathrm{pH}$ and salines to shield the fluorescent dyes from charged impurities on the glass surface. Sealing the sample chamber with silicone grease prevents drying and resulted in long-term stable behavior.

The bleaching from higher excited states is especially devastating, as it significantly reduces the total amount of photons that can be acquired: The electrons may cycle repeatedly between the first-order and higher excited states without emitting a single photon. As a linear dependence of the bleaching rate on the excitation intensity was observed for all experiments in this thesis, it can be concluded that the excitation powers used do not contribute to bleaching from higher excited states.

The bleaching experiments on single molecules were designed in a similar manner as described by Vogelsang et al. [53]: To avoid dye-dye interactions, surface effects [99] and orientation bias, single Atto647N dye molecules were attached to a double-stranded DNA string of 30 base pairs ( $10 \mathrm{~nm}$ total length), which was immobilized on the cover glass with a density $>1 \mu \mathrm{m}^{2}$. However, as is shown in Figure A.11a, the fluorophores can have distinct states exhibiting different brightness and STED efficiency. This might be caused by interactions of the dye with the DNA molecule or glass surface, and may account for the strong scattering of the bleaching data.

Vogelsang et al. studied the effects of various reagents on the blinking and bleaching kinetics of several dyes, including Atto647N. Adding either ascorbic acid as reducing agent or methyl-viologen as oxidizer to the oxygen-depleted PBS-buffer decreased the photon yield significantly. Adding both reagents simultaneously, however, increased the photon yield by orders of magnitude. These observations were explained by a strongly increased triplet lifetime of the dye when oxygen is removed, leading to reversible, yet reactive, photoinduced ionization. Oxidizing and reducing agents therefore need to effectively quench the triplet and ion states by electron transfer. Less blinking and increased photostability is only achieved when anionic and cationic molecules are neutralized Their work underlines that photobleaching does not follow a single path, yet may be caused by several reactions. The reported blinking behavior of Atto647N in PBS qualitatively agrees with the observed behavior in my single molecule measurements.

To my knowledge, no detailed analysis of photobleaching in STED microscopy has been published so far. Dyba and Hell [100] measured the bleaching dynamics of the dye RH-414 diffusing in a membrane, observing the reduction of the fluorescence intensity and subsequent recovery when the STED laser was turned on and off. The method has the advantage 
of being easy to implement and allows a direct observation of the photobleaching. However, it cannot yield accurate figures for the bleaching rate: The signal observed is emitted from a diffraction-limited spot with inhomogeneous illumination, and dyes diffusing through the focus are irradiated at various intensities before bleaching. Furthermore, low bleaching rates (lifetime $>$ diffusion time through the focus) are hard to observe.

Dyba and Hell showed that the bleaching rate is strongly dependent on the STED pulse length, suggesting a two-photon absorption process to be involved. Using a pulsed laser system, they also observed bleaching when the STED pulse acts before the arrival of the excitation pulse, indicating a similar bleaching mechanism as in my experiments. However, they attributed the STED bleaching to excitation from the ground state $S_{0}$ into higher excited states $S_{n}$ by the STED laser. This is backed by their observation that the bleaching does not change with the excitation power.

In contrast, as described in the results section, I conclude that the bleaching of Atto647N by the STED laser is primarily caused by electrons in the excited states absorbing a STED photon. This is based on two main differences in the observations: Where Dyba and Hell reported a non-linear dependency of the bleaching rate on the STED power, I observed a linear relationship. Furthermore, if bleaching by the excitation and STED laser were decoupled, one would expect a constant proportionality factor between bleaching rate and excitation power at different STED powers. The data in Figure 4.7a shows that the factor scales roughly linear with the STED power. Although bleaching by the STED laser in the absence of excitation was observed, it was considerably lower than with excitation and, most importantly, depended on the excitation power illuminating the molecule directly before the STED measurement. This strongly indicates that the main bleaching pathway includes a metastable excited state that is populated through the excitation laser.

Direct measurements of the bleaching rate using the STED laser alone have not been carried out. One would need to probe the dye with the excitation laser to check whether it is still fluorescent, followed by several milliseconds without irradiation to allow dark states to relax before illuminating with the STED laser, repeating the cycle until the molecule is bleached. Such an experiment would result in very long acquisition times making it vulnerable to sample drift. It was not carried out, reasoning that the observed bleaching behavior offers sufficient evidence for negligible STED bleaching from the $S_{0}$ state.

A very similar experiment to mine has been described by van Dijk et al. [98]. They investigated the bleaching of a fluorescent probe in an optical trap. While the trap laser wavelength was too long to cause stimulated emission, they observed a linear relationship of the bleaching rate to the laser power. No bleaching was observed when the excitation laser was switched off.

In a study published very recently, $\mathrm{Wu}$ et al. [50] investigated the bleaching behavior of Atto647N and other dyes in STED measurements at various scanning speeds. They derived a very similar bleaching model, concluding that the STED laser does not cause bleaching in the absence of the excitation laser. They have also observed a protective STED effect on Abberior Star Red, but not on Atto647N. As they used a different sample medium, STED laser wavelength and excitation laser powers, small differences in the experimental results are not surprising.

Considering the experimental evidence and literature, it can be concluded that the bleaching induced by the STED laser on Atto647N is caused by exciting electrons from the triplet and other long-lived states into higher excited states, which have a high probability of pho- 


\section{Discussion}

tobleaching. The model used for the bleaching behavior is widely used in the literature of this field and could adequately explain the results for photobleaching without stimulated emission. However, it fails when the STED laser pulse follows the excitation pulse within a few nanoseconds, thus being able to act on electrons in the $S_{1}$ state. The fast depletion of the excited states has been shown to increase the fluorophore's lifetime in STED microscopy $[50,101]$ and could be observed here on Atto647N and Star Red. One other effect that may be caused by the STED laser is reversible intersystem crossing (ISC) from the $\mathrm{T}_{n}$ to $\mathrm{S}_{n}$ states [102]. This would be possible also when the STED laser is not in synchrony with the excitation laser, which was not observed.

The unmodified bleaching model (equation (4.23)) would therefore predict that the stimulated emission through the STED laser prevents all bleaching from excited states. The additional bleaching must therefore be caused by $S_{1} \rightarrow S_{n}$ excitation by the STED laser, or a mechanism must exist that populates the triplet states despite the shortened lifetime of the $\mathrm{S}_{1}$ state. The former case is improbable, as the stimulated emission and excitation rate both scale linearly with the laser power and would therefore cancel out, unless the excitation is a two-photon process. One mechanism for the latter suggestion may be a very high ISC rate when the molecule is in the higher vibrational states, immediately after excitation. It has been reported that ISC is strongly coupled to the vibrational states [82], yet no further supporting or refuting literature on the speculation of fast ISC was found. An indication for such a process would be a wavelength dependent ISC rate. It might also be that an excitation to $S_{n}$ states by the STED laser and subsequent ISC is a responsible mechanism. This STED-laser induced ISC would be detectable through a decrease in fluorescence when alternating between STED and normal illumination faster than the triplet lifetime. However, such a fluorescence decrease should have been noticed in fast scanning systems $[50,103]$. As no process explaining the further bleaching by the STED laser could be identified, a simple saturation factor was introduced to the model that compensates the $S_{1}$ depopulation at high STED power (equation (4.24)). Further experiments are necessary to understand the bleaching pathway in detail.

Although the bleaching model is not as accurate as desired, it quantitatively describes the bleaching behavior of Atto647N in STED imaging. The measurements with Star Red show qualitatively similar results, yet are not in quantitative agreement with the model obtained from Atto647N.

\section{Small Scanning Fields}

It was demonstrated that reducing the scan area to below the diffraction limit can increase the signal yield more than 100-fold. This is a vast improvement that can be combined with other methods to reduce the bleaching:

- Optimizing the sample buffer can reduce the bleaching rate several-fold [104]

- Dark state relaxation (D-Rex) STED microscopy can increase the signal yield by up to 5-fold [105].

- Reducing the state transition cycles has been shown to reduce the bleaching rate up to 8 -fold [47] 
Especially the reduction of state transition cycles by further confining the scan area is promising: The electro-optic deflectors enable arbitrarily shaped scan patterns. A first overview scan on a small field with low dwell time or low STED power can already pre-locate the labeled structure, e.g. a nuclear pore. A second scan with high resolution then scans only the areas of interest. Alternatively, an electro-optic modulator is used to blank the lasers at spots with no dye. The difference to the method described in [47] is that one can choose the scan / blanking pattern based on the information of whole image, instead of choosing to blank the lasers on a pixel by pixel basis. This approach is difficult to apply to larger scan fields, because sample drift compromises the information of a first scan, and line-scanning with different dwell times will result in different scan position lags, thus complicating an accurate pre-location.

Further increasing the resolution beyond the results shown in this thesis is a matter of increasing the STED laser power. Here, the average power coupled through the deflectors and into the objective already exceeds the recommended maximum values. The maximum laser power used in this thesis was $\approx 700 \mathrm{~mW}$ at the objective. It was observed, that the heating of the components through the laser causes a significant sample drift, blurring the final image. Implementing a laser with a lower repetition rate will allow higher pulse energies to be used, while also protecting the dyes through D-Rex, enabling higher resolution.

When the scan area is adapted to the resolution, the attainable imaging resolution is not limited by the bleaching of the dye anymore. Consider a dye molecule being imaged with a small field of view. One can assume that the excitation intensity it is receiving during the scan stays constant, and the STED power scales quadratically with the distance of the doughnut zero to the dye. Doubling the STED laser power will improve the resolution by $\sqrt{2}$. Reducing the scan area size (length of the square area) by $\sqrt{2}$ and keeping the number of pixels constant, will resolve the same amount of detail relative to the area size as before. The irradiation received by the dye does not change in this scenario, as the maximal distance between dye and doughnut zero is reduced by $\sqrt{2}$, i.e. the maximal STED intensity is $2 / 2$ of the initial intensity. The bleaching rate, and therefore the attainable signal, is unchanged.

\section{Two-Color STED}

The two-color STED microscope built was the next iteration step in line with previously developed setups, combining existing methods to provide reliable performance, high accuracy and viability. Common problems of two-color STED microscopes were tackled: The necessity for sequential imaging [37,39] and low co-alignment accuracy [38] is avoided by using spectrally close dyes that allow resolution improvement by STED with a single laser [40]. The spectral crosstalk between the color channels $[40,41]$ is reduced using pulse-interleaved excitation and detection [38].

The setup proved to be a stable and reliable instrument, leading to many successful and ongoing cooperations. We could show its viability for a large range of sample types. Novel cell permeable dyes enabled live cell measurements [106,107], while the long STED wavelength and long pulse lengths minimized phototoxic effects. STED microscopy of different sample types such as melamine embedded sections [107] and tissue [108, 109, 110, 111] is possible without technical alterations to the setup.

However, one would often desire a higher signal-to-noise ratio and better resolution for 


\section{Discussion}

many applications. As the dyes used in the small area setup are the same as the far-red dyes the two-color setup is optimized for, implementing a second color channel into the former setup is straight-forward. Many structures investigated on the two-color setup (e.g. nuclear pores [54], synapses [108, 109, 110, 111], centrioles [106]) are in the size order of $200 \mathrm{~nm}$ and are sparsely distributed, thus making good targets for the small area scans.

\section{Future Applications}

I would like to conclude this thesis with an outlook to further projects that can profit from small scan areas, but have not yet been pursued. The fundamental concept of reducing the bleaching rate in the field of view by avoiding the irradiation when measuring adjacent areas is applicable to all targeted super-resolution methods, for example RESOLFT with photo-switchable proteins. The signal yield can be greatly increased for a large number of methods without affecting the processes involved in the image formation, thus increasing the information obtainable from the sample or enabling higher resolution. One application of high interest in a biological context is molecule counting [51], which requires a high photon yield to produce accurate numbers.

The primary reason I used an electro-optic scanning system is the necessarily high framerate of the acquisition, which is a result of the few sampling points in a small image. An image with $10 \times 10$ pixels is usually acquired withing $10 \mathrm{~ms}$, not compromising on the signal acquisition time. The high frame-rate enables the imaging of dynamic processes in a cell, while at the same time the photobleaching is delayed through the small scan area. This combination should allow interesting insights into the nanoscale dynamics of otherwise stationary structures, e.g. nuclear pores and synapses.

In conclusion, I developed a method that can increase the signal yield in RESOLFT superresolution microscopy by more than a factor of 100. It is a promising tool for a wide range of biological applications. The major limitation is the inevitable small field of view of less than $200 \times 200 \mathrm{~nm}^{2}$, requiring an intelligent and precise positioning of the scanning field through specialized software. Implementing this method in a two-color STED microscope poses no further technical challenges and will follow in the near future, opening its advantages for ongoing projects in biology. 


\section{Bibliography}

[1] Bradbury S. The evolution of the microscope. Pergamon Press, New York; 1967.

[2] Volkmann H. Ernst Abbe and his work. Applied Optics. 1966;5(11):1720-1731.

[3] Paul H. Lexikon der Optik. Spektrum Akademischer Verlag, Heidelberg; 1999.

[4] Pulizzi F, editor. Electron and X-ray microscopy. Nature Materials. 2009;8(4).

[5] Pohl DW, Denk W, Lanz M. Optical stethoscopy: Image recording with resolution $\lambda / 20$. Applied Physics Letters. 1984;44(7):651-653.

[6] Binnig G, Quate CF, Gerber C. Atomic force microscope. Physical Review Letters. 1986;56(9):930-934.

[7] Binnig G, Rohrer H, Gerber C, Weibel E. Surface studies by scanning tunneling microscopy. Physical Review Letters. 1982;49(1):57-61.

[8] Stryer L, Haugland RP. Energy transfer: a spectroscopic ruler. Proceedings of the National Academy of Sciences of the United States of America. 1967;58(2):719-726.

[9] Magde D, Elson E, Webb WW. Thermodynamic fluctuations in a reacting system - measurement by fluorescence correlation spectroscopy. Physical Review Letters. 1972;29(11):705-708.

[10] Otosu T, Ishii K, Tahara T. Microsecond protein dynamics observed at the single-molecule level. Nature Communications. 2015;6:7685.

[11] Hell SW, Wichmann J. Breaking the diffraction resolution limit by stimulated emission: stimulated-emission-depletion fluorescence microscopy. Optics Letters. 1994;19(11):780782.

[12] Harke B, Keller J, Ullal CK, Westphal V, Schönle A, Hell SW. Resolution scaling in STED microscopy. Optics Express. 2008;16(6):4154-4162.

[13] Wildanger D, Patton BR, Schill H, Marseglia L, Hadden J, Knauer S, Schönle A, Rarity JG, O’Brien JL, Hell SW, Smith JM. Solid immersion facilitates fluorescence microscopy with nanometer resolution and sub-Ångström emitter localization. Advanced Materials. 2012;24(44):OP309-OP313.

[14] Airy GB. On the diffraction of an object-glass with circular aperture. Transactions of the Cambridge Philosophical Society. 1835;5:283-291.

[15] Sibarita JB. Deconvolution microscopy. In: Rietdorf J, editor. Microscopy techniques. vol. 95 of Advances in Biochemical Engineering. Springer, Heidelberg; 2005. p. 201-243. 


\section{BIBLIOGRAPHY}

[16] Cox G, Sheppard CJ. Practical limits of resolution in confocal and non-linear microscopy. Microscopy Research and Technique. 2004;63(1):18-22.

[17] Hell S, Stelzer EH. Properties of a 4Pi confocal fluorescence microscope. Journal of the Optical Society of America A. 1992;9(12):2159-2166.

[18] Gustafsson, Agard, Sedat. I5M: 3D widefield light microscopy with better than $100 \mathrm{~nm}$ axial resolution. Journal of Microscopy. 1999;195(1):10-16.

[19] Gustafsson MG. Surpassing the lateral resolution limit by a factor of two using structured illumination microscopy. Journal of Microscopy. 2000;198(2):82-87.

[20] Heintzmann R, Jovin TM, Cremer C. Saturated patterned excitation microscopy - a concept for optical resolution improvement. Journal of the Optical Society of America A. 2002;19(8):1599-1609.

[21] Gustafsson MG. Nonlinear structured-illumination microscopy: wide-field fluorescence imaging with theoretically unlimited resolution. Proceedings of the National Academy of Sciences of the United States of America. 2005;102(37):13081-13086.

[22] Gazit S, Szameit A, Eldar YC, Segev M. Super-resolution and reconstruction of sparse sub-wavelength images. Optics Express. 2009;17(26):23920-23946.

[23] Sarder P, Nehorai A. Deconvolution methods for 3-D fluorescence microscopy images. Signal Processing Magazine, IEEE. 2006;23(3):32-45.

[24] Hell SW. Microscopy and its focal switch. Nature Methods. 2009;6(1):24-32.

[25] Rust MJ, Bates M, Zhuang X. Sub-diffraction-limit imaging by stochastic optical reconstruction microscopy (STORM). Nature Methods. 2006;3(10):793-796.

[26] Hess ST, Girirajan TP, Mason MD. Ultra-high resolution imaging by fluorescence photoactivation localization microscopy. Biophysical Journal. 2006;91(11):4258-4272.

[27] Betzig E, Patterson GH, Sougrat R, Lindwasser OW, Olenych S, Bonifacino JS, Davidson MW, Lippincott-Schwartz J, Hess HF. Imaging intracellular fluorescent proteins at nanometer resolution. Science. 2006;313(5793):1642-1645.

[28] Wurm CA, Kolmakov K, Göttfert F, Ta H, Bossi M, Schill H, Berning S, Jakobs S, Donnert G, Belov VN, Hell SW. Novel red fluorophores with superior performance in STED microscopy. Optical Nanoscopy. 2012;1(1):1-7.

[29] Reindl S, Penzkofer A. Triplet quantum yield determination by picosecond laser doublepulse fluorescence excitation. Chemical Physics. 1996;213(1):429-438.

[30] Reindl S, Penzkofer A. Higher excited-state triplet-singlet intersystem crossing of some organic dyes. Chemical Physics. 1996;211(1):431-439.

[31] Kasha M. Characterization of electronic transitions in complex molecules. Discussions of the Faraday Society. 1950;9:14-19.

[32] Lakowicz JR. Principles of fluorescence spectroscopy. 3rd ed. Springer, Heidelberg; 2006. 
[33] Willig KI, Harke B, Medda R, Hell SW. STED microscopy with continuous wave beams. Nature Methods. 2007;4(11):915-918.

[34] Bingen P, Reuss M, Engelhardt J, Hell SW. Parallelized STED fluorescence nanoscopy. Optics Express. 2011;19(24):23716-23726.

[35] Leutenegger M, Eggeling C, Hell SW. Analytical description of STED microscopy performance. Optics Express. 2010;18(25):26417-26429.

[36] Keller J, Schönle A, Hell SW. Efficient fluorescence inhibition patterns for RESOLFT microscopy. Optics Express. 2007;15(6):3361-3371.

[37] Donnert G, Keller J, Wurm CA, Rizzoli SO, Westphal V, Schönle A, Jahn R, Jakobs S, Eggeling C, Hell SW. Two-color far-field fluorescence nanoscopy. Biophysical Journal. 2007;92(8):L67-L69.

[38] Bückers J, Wildanger D, Vicidomini G, Kastrup L, Hell SW. Simultaneous multi-lifetime multi-color STED imaging for colocalization analyses. Optics Express. 2011;19(4):31303143.

[39] Meyer L, Wildanger D, Medda R, Punge A, Rizzoli SO, Donnert G, Hell SW. Dual-color STED microscopy at 30-nm focal-plane resolution. Small. 2008;4(8):1095-1100.

[40] Schmidt R, Wurm CA, Jakobs S, Engelhardt J, Egner A, Hell SW. Spherical nanosized focal spot unravels the interior of cells. Nature Methods. 2008;5(6):539-544.

[41] Tønnesen J, Nadrigny F, Willig KI, Wedlich-Söldner R, Nägerl UV. Two-color STED microscopy of living synapses using a single laser-beam pair. Biophysical Journal. 2011;101(10):2545-2552.

[42] Willig KI, Stiel AC, Brakemann T, Jakobs S, Hell SW. Dual-label STED nanoscopy of living cells using photochromism. Nano Letters. 2011;11(9):3970-3973.

[43] Berning S. STED nanoscopy of the living brain. Dissertation, Georg-August Universität Göttingen; 2011. Urn:nbn:de:gbv:7-webdoc-3491-0.

[44] Pawley JB. Fundamental limits in confocal microscopy. In: Handbook of biological confocal microscopy. Springer, Heidelberg; 2006. p. 20-42.

[45] Ono M, Murakami T, Kudo A, Isshiki M, Sawada H, Segawa A. Quantitative comparison of anti-fading mounting media for confocal laser scanning microscopy. Journal of Histochemistry \& Cytochemistry. 2001;49(3):305-311.

[46] Donnert G, Keller J, Medda R, Andrei MA, Rizzoli SO, Lührmann R, Jahn R, Eggeling C, Hell SW. Macromolecular-scale resolution in biological fluorescence microscopy. Proceedings of the National Academy of Sciences of the United States of America. 2006;103(31):11440-11445.

[47] Staudt T, Engler A, Rittweger E, Harke B, Engelhardt J, Hell SW. Far-field optical nanoscopy with reduced number of state transition cycles. Optics Express. 2011;19(6):5644-5657. 


\section{BIBLIOGRAPHY}

[48] Schneider J, Zahn J, Maglione M, Sigrist SJ, Marquard J, Chojnacki J, Kräusslich HG, Sahl SJ, Engelhardt J, Hell SW. Ultrafast, temporally stochastic STED nanoscopy of millisecond dynamics. Nature Methods. 2015;p. 827-830.

[49] Schneider J. Entwicklung eines ultraschnellen elektro-optischen Scanners für STEDNanoskopie. Dissertation, RWTH Aachen; 2013. Urn:nbn:de:hbz:82-opus-46203.

[50] Wu Y, Wu X, Lu R, Zhang J, Toro L, Stefani E. Resonant scanning with large field of view reduces photobleaching and enhances fluorescence yield in STED microscopy. Scientific Reports. 2015;5:14766-14766.

[51] Ta H, Keller J, Haltmeier M, Saka SK, Schmied J, Opazo F, Tinnefeld P, Munk A, Hell SW. Mapping molecules in scanning far-field fluorescence nanoscopy. Nature Communications. 2015;6:7977.

[52] Heilemann M, Kasper R, Tinnefeld P, Sauer M. Dissecting and reducing the heterogeneity of excited-state energy transport in DNA-based photonic wires. Journal of the American Chemical Society. 2006;128(51):16864-16875.

[53] Vogelsang J, Kasper R, Steinhauer C, Person B, Heilemann M, Sauer M, Tinnefeld P. A reducing and oxidizing system minimizes photobleaching and blinking of fluorescent dyes. Angewandte Chemie International Edition. 2008;47(29):5465-5469.

[54] Göttfert F, Wurm CA, Mueller V, Berning S, Cordes VC, Honigmann A, Hell SW. Coaligned dual-channel STED nanoscopy and molecular diffusion analysis at $20 \mathrm{~nm}$ resolution. Biophysical Journal. 2013;105(1):L01-L03.

[55] Pleiner T, Bates M, Trakhanov S, Lee CT, Schliep JE, Chug H, Böhning M, Stark H, Urlaub H, Görlich D. Nanobodies: site-specific labeling for super-resolution imaging, rapid epitope-mapping and native protein complex isolation. eLife. 2015;4:e11349.

[56] Henrich MB. The potential of semiconductor lasers for STED nanoscopy. Dissertation, Universität Heidelberg; 2014. Urn:nbn:de:bsz:16-heidok-169070.

[57] Müller BK, Zaychikov E, Bräuchle C, Lamb DC. Pulsed interleaved excitation. Biophysical Journal. 2005;89(5):3508-3522.

[58] Vicidomini G, Moneron G, Han KY, Westphal V, Ta H, Reuss M, Engelhardt J, Eggeling C, Hell SW. Sharper low-power STED nanoscopy by time gating. Nature Methods. 2011;8(7):571-573.

[59] Schönle A. Imspector Image Acquisition E Analysis Software, v0.10; 2006. http://www.imspector.de.

[60] Rittweger E, Rankin B, Westphal V, Hell S. Fluorescence depletion mechanisms in superresolving STED microscopy. Chemical Physics Letters. 2007;442(4):483-487.

[61] Hess ST, Huang S, Heikal AA, Webb WW. Biological and chemical applications of fluorescence correlation spectroscopy: a review. Biochemistry. 2002;41(3):697-705.

[62] Thompson NL. Fluorescence correlation spectroscopy. In: Lakowicz JR, editor. Topics in fluorescence spectroscopy, vol. 1. Springer, Heidelberg; 1999. p. 337-378. 
[63] Eggeling C, Ringemann C, Medda R, Schwarzmann G, Sandhoff K, Polyakova S, Belov VN, Hein B, von Middendorff C, Schönle A, Hell SW. Direct observation of the nanoscale dynamics of membrane lipids in a living cell. Nature. 2009;457(7233):1159-1162.

[64] Schwille P, Meyer-Almes FJ, Rigler R. Dual-color fluorescence cross-correlation spectroscopy for multicomponent diffusional analysis in solution. Biophysical Journal. 1997;72(4):1878-1886.

[65] Honigmann A, Mueller V, Hell SW, Eggeling C. STED microscopy detects and quantifies liquid phase separation in lipid membranes using a new far-red emitting fluorescent phosphoglycerolipid analogue. Faraday Discussions. 2013;161:77-89.

[66] Maimon T, Elad N, Dahan I, Medalia O. The human nuclear pore complex as revealed by cryo-electron tomography. Structure. 2012;20(6):998-1006.

[67] Szymborska A, de Marco A, Daigle N, Cordes VC, Briggs JA, Ellenberg J. Nuclear pore scaffold structure analyzed by super-resolution microscopy and particle averaging. Science. 2013;341(6146):655-658.

[68] Löschberger A, van de Linde S, Dabauvalle MC, Rieger B, Heilemann M, Krohne G, Sauer M. Super-resolution imaging visualizes the eightfold symmetry of gp 210 proteins around the nuclear pore complex and resolves the central channel with nanometer resolution. Journal of Cell Science. 2012;125(3):570-575.

[69] Cohen M, Feinstein N, Wilson KL, Gruenbaum Y. Nuclear pore protein gp210 is essential for viability in HeLa cells and Caenorhabditis elegans. Molecular Biology of the Cell. 2003;14(10):4230-4237.

[70] Hoelz A, Debler EW, Blobel G. The structure of the nuclear pore complex. Annual Review of Biochemistry. 2011;80:613-643.

[71] Marshall GF, Stutz GE. Handbook of optical and laser scanning. 2nd ed. CRC Press, Boca Raton; 2012.

[72] Python Software Foundation. http://www.python.org.

[73] Peterson P. PyLibNIDAQmx: a Python wrapper to libnidaqmx library; 2009. https://github.com/pearu/pylibnidaqmx.

[74] The HDF Group. Hierarchical Data Format, version 5; 1997-. http://www.hdfgroup.org/HDF5/.

[75] The Qt Company. http://www.qt.io.

[76] Qt Project. http://www.pyside.org/.

[77] Goldberg IG, Allan C, Burel JM, Creager D, Falconi A, Hochheiser H, Johnston J, Mellen J, Sorger PK, Swedlow JR. The open microscopy environment (OME) data model and XML file: open tools for informatics and quantitative analysis in biological imaging. Genome Biology. 2005;6(5):R47. 


\section{BIBLIOGRAPHY}

[78] Linkert M, Rueden CT, Allan C, Burel JM, Moore W, Patterson A, Loranger B, Moore J, Neves C, MacDonald D, Tarkowska A, Sticco C, Hill e, Rossner M, Eliceiri KW, Swedlow JR. Metadata matters: access to image data in the real world. The Journal of Cell Biology. 2010;189(5):777-782.

[79] Zondervan R, Kulzer F, Kol'chenk MA, Orrit M. Photobleaching of rhodamine 6G in poly (vinyl alcohol) at the ensemble and single-molecule levels. The Journal of Physical Chemistry A. 2004;108(10):1657-1665.

[80] Eggeling C, Widengren J, Rigler R, Seidel C. Photostability of fluorescent dyes for singlemolecule spectroscopy: mechanisms and experimental methods for estimating photobleaching in aqueous solution. In: Rettig W, Strehmel B, Schrader S, Seifert H, editors. Applied fluorescence in chemistry, biology and medicine. Springer, Heidelberg; 1999. p. 193-240.

[81] ATTO-TEC. Dye specifications.

[82] Lawetz V, Orlandi G, Siebrand W. Theory of intersystem crossing in aromatic hydrocarbons. The Journal of Chemical Physics. 1972;56(8):4058-4072.

[83] Rothemund PW. Folding DNA to create nanoscale shapes and patterns. Nature. 2006;440(7082):297-302.

[84] Schmied JJ, Gietl A, Holzmeister P, Forthmann C, Steinhauer C, Dammeyer T, Tinnefeld P. Fluorescence and super-resolution standards based on DNA origami. Nature Methods. 2012;9(12):1133-1134.

[85] Vira S, Mekhedov E, Humphrey G, Blank PS. Fluorescent-labeled antibodies: Balancing functionality and degree of labeling. Analytical Biochemistry. 2010;402(2):146-150.

[86] Aquino D, Schönle A, Geisler C, v Middendorff C, Wurm CA, Okamura Y, Lang T, Hell SW, Egner A. Two-color nanoscopy of three-dimensional volumes by 4Pi detection of stochastically switched fluorophores. Nature Methods. 2011;8(4):353-359.

[87] Hamers-Casterman C, Atarhouch T, Muyldermans S, Robinson G, Hammers C, Songa EB, Bendahman N, Hammers R. Naturally occurring antibodies devoid of light chains. Nature. 1993;363(6428):446-448.

[88] Harmsen M, De Haard H. Properties, production, and applications of camelid singledomain antibody fragments. Applied Microbiology and Biotechnology. 2007;77(1):1322.

[89] Chen RF, Knutson JR. Mechanism of fluorescence concentration quenching of carboxyfluorescein in liposomes: energy transfer to nonfluorescent dimers. Analytical Biochemistry. 1988;172(1):61-77.

[90] Luchowski R, Matveeva EG, Gryczynski I, Terpetschnig EA, Patsenker L, Laczko G, Borejdo J, Gryczynski Z. Single molecule studies of multiple-fluorophore labeled antibodies. Effect of homo-FRET on the number of photons available before photobleaching. Current Pharmaceutical Biotechnology. 2008;9(5):411-420. 
[91] Song L, Hennink E, Young IT, Tanke HJ. Photobleaching kinetics of fluorescein in quantitative fluorescence microscopy. Biophysical Journal. 1995;68(6):2588-2600.

[92] Deschenes LA, Bout DAV. Single molecule photobleaching: increasing photon yield and survival time through suppression of two-step photolysis. Chemical Physics Letters. 2002;365(5):387-395.

[93] Eggeling C, Widengren J, Rigler R, Seidel C. Photobleaching of fluorescent dyes under conditions used for single-molecule detection: Evidence of two-step photolysis. Analytical Chemistry. 1998;70(13):2651-2659.

[94] Eggeling C, Volkmer A, Seidel CA. Molecular photobleaching kinetics of rhodamine $6 G$ by one-and two-photon induced confocal fluorescence microscopy. ChemPhysChem. 2005;6(5):791-804.

[95] Song L, Varma C, Verhoeven J, Tanke HJ. Influence of the triplet excited state on the photobleaching kinetics of fluorescein in microscopy. Biophysical Journal. 1996;70(6):29592968.

[96] Niesner R, Roth W, Gericke KH. Photophysical aspects of single-molecule detection by two-photon excitation with consideration of sequential pulsed illumination. ChemPhysChem. 2004;5(5):678-687.

[97] Hoogenboom JP, van Dijk EM, Hernando J, van Hulst NF, García-Parajó MF. Powerlaw-distributed dark states are the main pathway for photobleaching of single organic molecules. Physical Review Letters. 2005;95(9):097401.

[98] van Dijk MA, Kapitein LC, van Mameren J, Schmidt CF, Peterman EJ. Combining optical trapping and single-molecule fluorescence spectroscopy: Enhanced photobleaching of fluorophores. The Journal of Physical Chemistry B. 2004;108(20):6479-6484.

[99] Weston KD, Carson PJ, Metiu H, Buratto SK. Room-temperature fluorescence characteristics of single dye molecules adsorbed on a glass surface. The Journal of Chemical Physics. 1998;109(17):7474-7485.

[100] Dyba M, Hell SW. Photostability of a fluorescent marker under pulsed excited-state depletion through stimulated emission. Applied Optics. 2003;42(25):5123-5129.

[101] Eggeling C, Willig KI, Sahl SJ, Hell SW. Lens-based fluorescence nanoscopy. Quarterly Reviews of Biophysics. 2015;48(02):178-243.

[102] Ringemann C, Schönle A, Giske A, von Middendorff C, Hell SW, Eggeling C. Enhancing fluorescence brightness: effect of reverse intersystem crossing studied by fluorescence fluctuation spectroscopy. ChemPhysChem. 2008;9(4):612-624.

[103] Westphal V, Rizzoli SO, Lauterbach MA, Kamin D, Jahn R, Hell SW. Video-rate far-field optical nanoscopy dissects synaptic vesicle movement. Science. 2008;320(5873):246-249.

[104] Kasper R, Harke B, Forthmann C, Tinnefeld P, Hell SW, Sauer M. Single-molecule STED microscopy with photostable organic fluorophores. Small. 2010;6(13):1379-1384. 


\section{BIBLIOGRAPHY}

[105] Donnert G, Eggeling C, Hell SW. Triplet-relaxation microscopy with bunched pulsed excitation. Photochemical \& Photobiological Sciences. 2009;8(4):481-485.

[106] Lukinavičius G, Reymond L, D’Este E, Masharina A, Göttfert F, Ta H, Güther A, Fournier M, Rizzo S, Waldmann H, Blaukopf C, Sommer C, Gerlich DW, Arndt HD, Hell SW, Johnsson K. Fluorogenic probes for live-cell imaging of the cytoskeleton. Nature Methods. 2014;11(7):731-733.

[107] D'Este E, Kamin D, Göttfert F, El-Hady A, Hell SW. STED nanoscopy reveals the ubiquity of subcortical cytoskeleton periodicity in living neurons. Cell Reports. 2015;10(8):1246-1251.

[108] Chapochnikov NM, Takago H, Huang CH, Pangršič T, Khimich D, Neef J, Auge E, Göttfert F, Hell SW, Wichmann C, Wolf F, Moser T. Uniquantal release through a dynamic fusion pore is a candidate mechanism of hair cell exocytosis. Neuron. 2014;83(6):13891403.

[109] Wong AB, Rutherford MA, Gabrielaitis M, Pangršič T, Göttfert F, Frank T, Michanski S, Hell S, Wolf F, Wichmann C, Moser T. Developmental refinement of hair cell synapses tightens the coupling of Ca2+ influx to exocytosis. The EMBO Journal. 2014;33(3):247264.

[110] Siebert M, Böhme MA, Driller JH, Babikir H, Mampell MM, Rey U, Ramesh N, Matkovic T, Holton N, Reddy-Alla S, Göttfert F, Kamin D, Quentin C, Klinedinst S, Andlauer TF, Hell SW, Collins CA, Wahl MC, Loll B, Sigrist SJ. A high affinity RIMbinding protein/Aplip1 interaction prevents the formation of ectopic axonal active zones. eLife. 2015;4:e06935.

[111] Jung S, Oshima-Takago T, Chakrabarti R, Wong AB, Jing Z, Yamanbaeva G, Picher MM, Wojcik SM, Göttfert F, Predoehl F, Micheli K, Hell SW, Schoch S, Strenzke N, Wichmann C, Moser T. Rab3-interacting molecules $2 \alpha$ and $2 \beta$ promote the abundance of voltage-gated $\mathrm{Ca}_{V} 1.3 \mathrm{Ca}^{2+}$ channels at hair cell active zones. Proceedings of the National Academy of Sciences of the United States of America. 2015;112(24):E3141-E3149. 


\section{A. Appendix}

\section{A.1. Resolution of the Two-Color Setup}

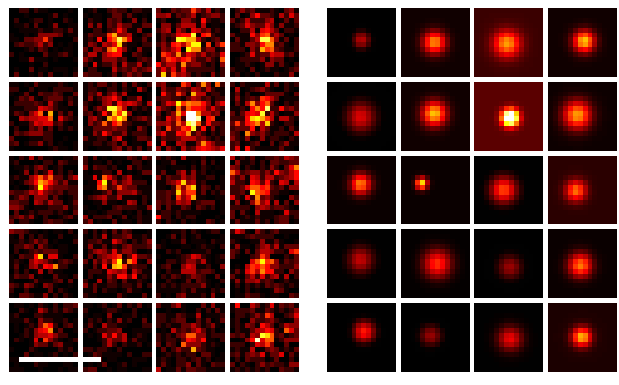

(a) Star635P

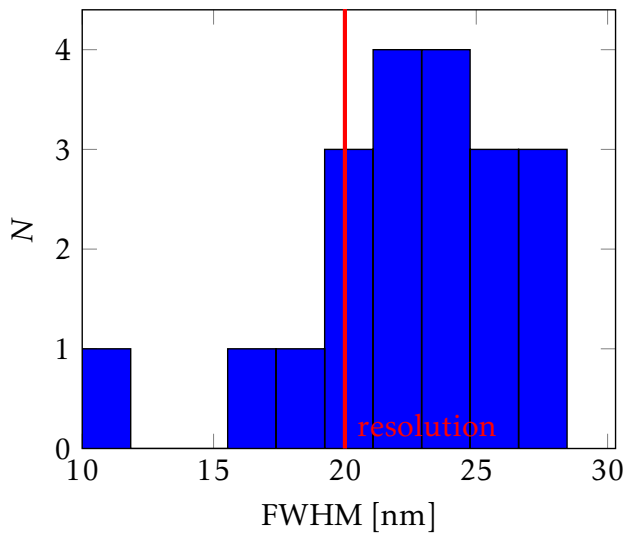

(c) Star635P

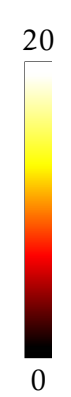

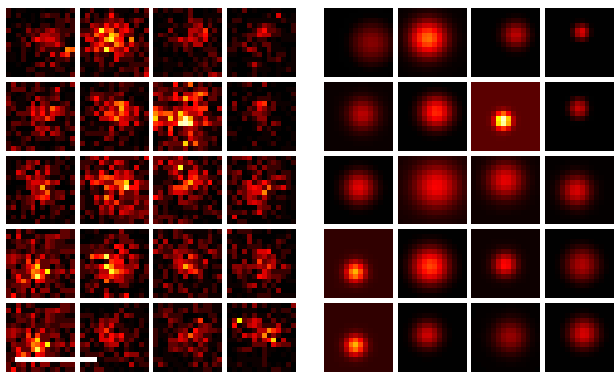

(b) Atto590

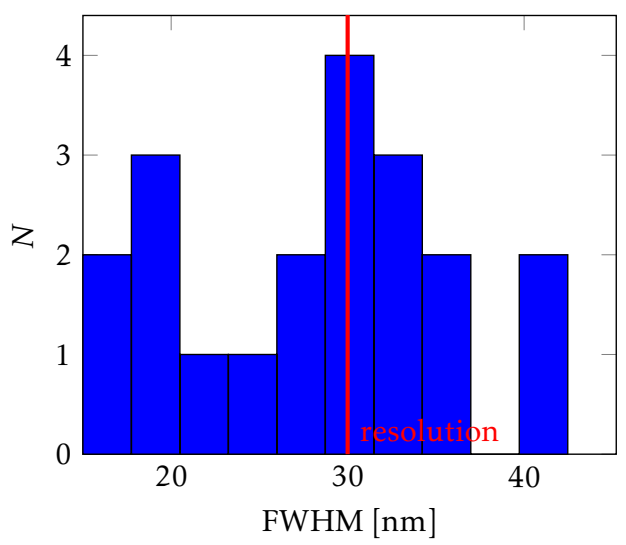

(d) Atto590

Figure A.1. The resolution in the two-color setup was determined by manually selecting small point-like structures in the images of gp210 / PanFG samples (Figure 3.7). (a): Star635P clusters (red channel) and fit with a 2D Gaussian. (b): Atto590 clusters (green channel) and fit with a 2D Gaussian. (c) and (d): Histogram of the FWHMs of above fits. 


\section{A.2. DNA Origami}

Additional plots and figures of the DNA origami data. The experiment is described in Section 4.3.1.

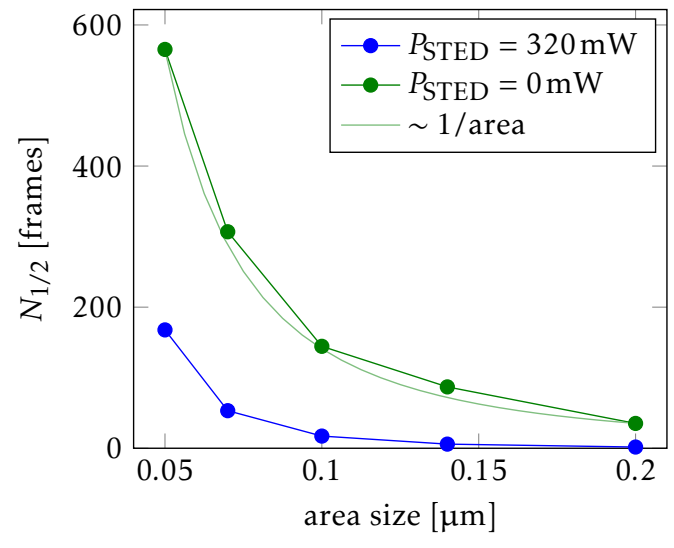

(a)

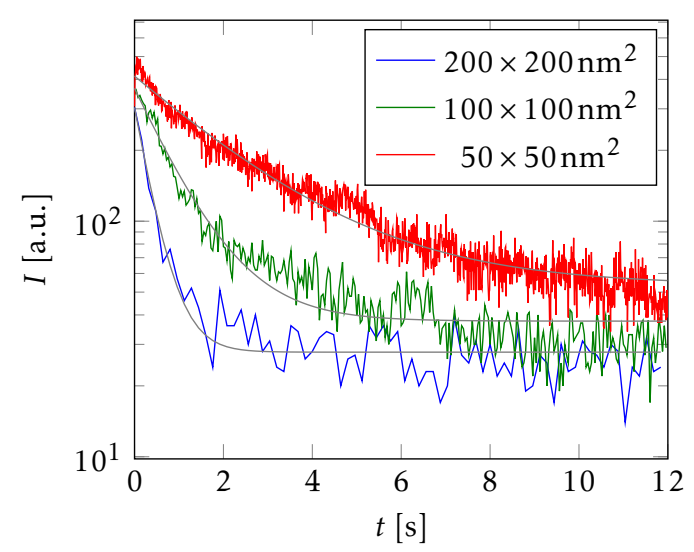

(c) STED data

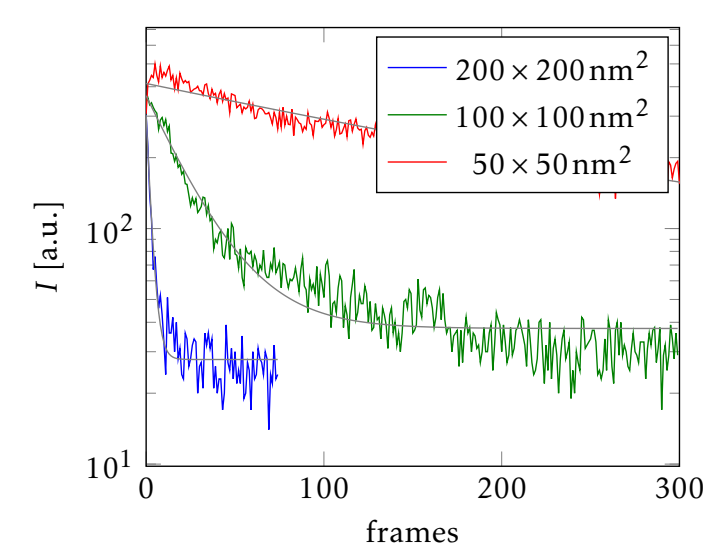

(b) STED data

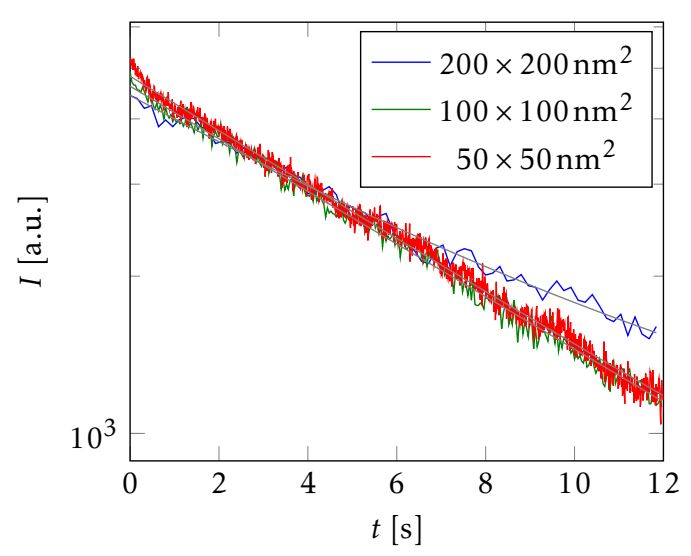

(d) confocal data

Figure A.2. Additional views of the data shown in Figure 4.10d. (a): Frames acquired until half the fluorescence is bleached. The light green curve is proportional to the inverse of the scan area. (b): Fluorescence signal decay of the STED measurements (c): Fluorescence signal decay of the STED measurements, versus measurement time. (d): Fluorescence signal decay of the confocal measurements, versus measurement time.

\begin{tabular}{|rlllll|}
\hline scan size [nm] & 200 & 140 & 100 & 70 & 50 \\
STED $N_{1 / 2}$ [frames] & 1.7 & 5.8 & 17.3 & 53.2 & 167.7 \\
confocal $N_{1 / 2}$ [frames] & 35.2 & 87.0 & 144.5 & 306.9 & 565.3 \\
\hline
\end{tabular}

Table A.1. Results for $N_{1 / 2}$ obtained by fitting an exponential decay to the fluorescence time trace (Figure A.2b). The pixel dwell time is $100 \mu$ s at a pixel size of $5 \mathrm{~nm}$. 
$t$

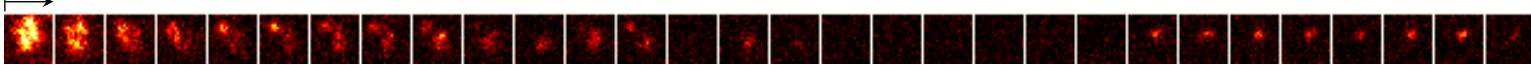

Figure A.3. It is already clear from figure Figure A.2 that the bleaching does not follow an exponential decay. The deviation is more obvious when looking at single origami structures: Pronounced blinking is observed. The above image shows a time series of a $100 \mathrm{~nm}$ scan with a frame rate of $1 / 400 \mathrm{~ms}$.

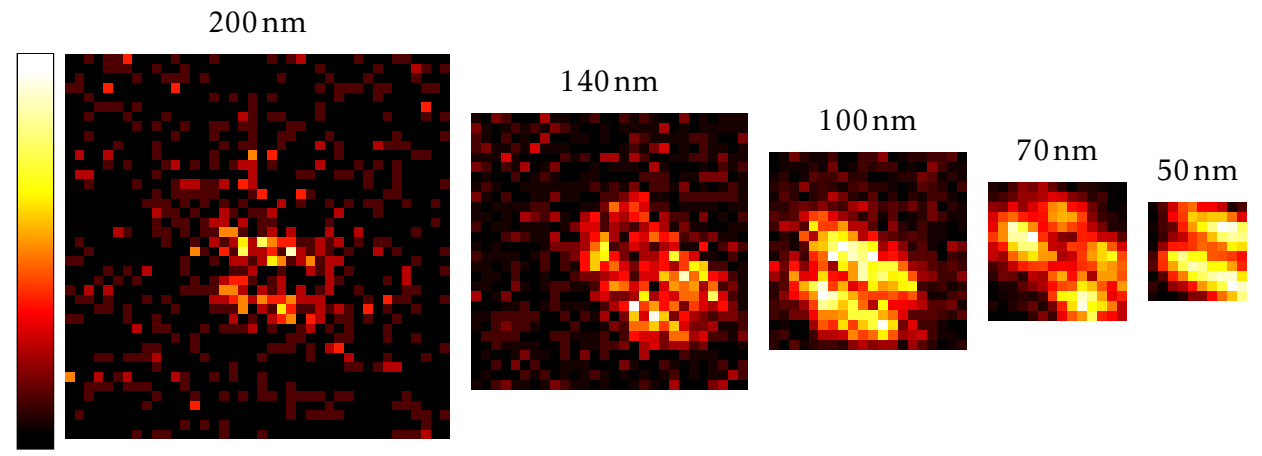

Figure A.4. Representative images of the DNA origami data, imaged with different field of views. The fluorescence signal has been summed over the first $N_{1 / 2}$ frames (rounded values, see Table A.1). Colorscale at (0-8), (0-17), (0-36), (5-103) and (37-299).

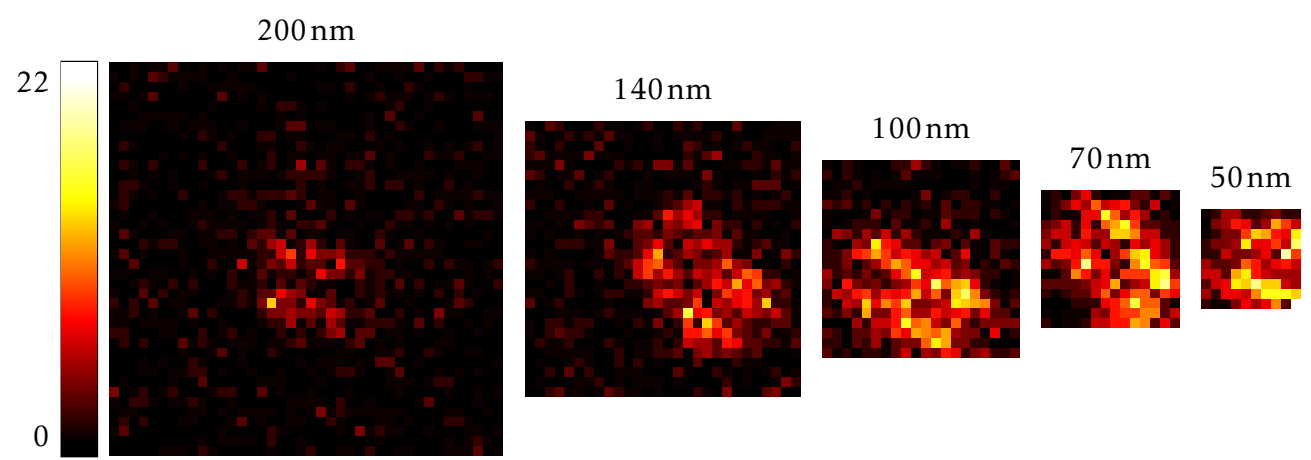

Figure A.5. The same data as in Figure A.4, summed over the first 5 frames. To illustrate that (i) the higher signal for the mall FOVs is not only due to the higher number of summed frames, and (ii) one has to be careful when deciding how long to integrate the signal, as some dyes bleach early while others have very long lifetimes, which results in artifacts (different signal distribution in the middle of the $70 \mathrm{~nm}$ image of Figure A.4). 


\section{A. Appendix}

\section{A.3. Single Molecule Bleaching}

Here, more details on the single molecule bleaching measurements are presented. As discussed in the main text, the bleaching data was not sufficiently accurate. Some possible error sources are addressed, yet no single cause for the unreliable data could be found.

The processing of the data is outlined in Figure A.6. The bleaching times can be obtained by either averaging the single molecule's bleaching times, or fitting an exponential decay to the summed signal. As can be seen in Figures A.7a and A.9, the results of both methods agree very well. The method of averaging however seems to have less extremes.

A measurement series was usually acquired during one week, thus the sample needs to be stable enough over this time period. That this is the case is shown in Figure A.7a. However, it becomes clear that the scattering of the bleaching rate in the short term is much larger than expected, although no measurement parameters were changed.

No correlation between the mean fluorescence and the bleaching time was observed. Thus, fluctuations in the laser power are not the cause for the scattering.

A possible error source may be bad positioning of the laser focus. The single-molecule STED measurements were carried out with the same positioning algorithm. As can be seen in Figure A.13, the positioning is quite accurate.

As the distribution of the dye's brightness and depletion efficiency is much broader than expected, the most likely error source is the heterogeneity of the dye behavior itself. 


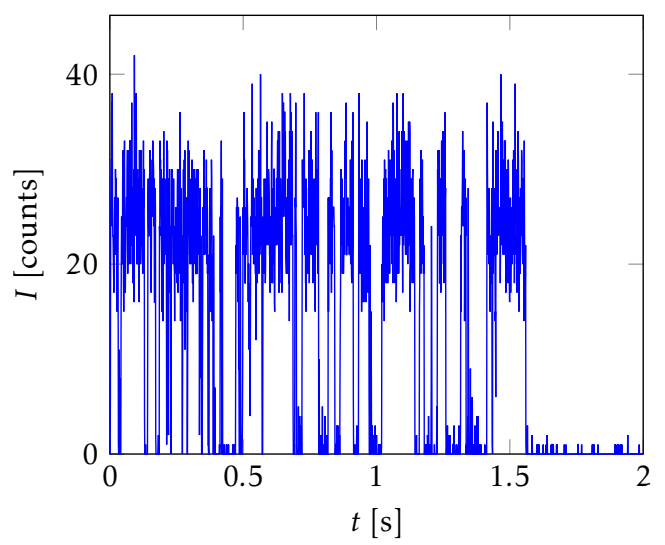

(a)

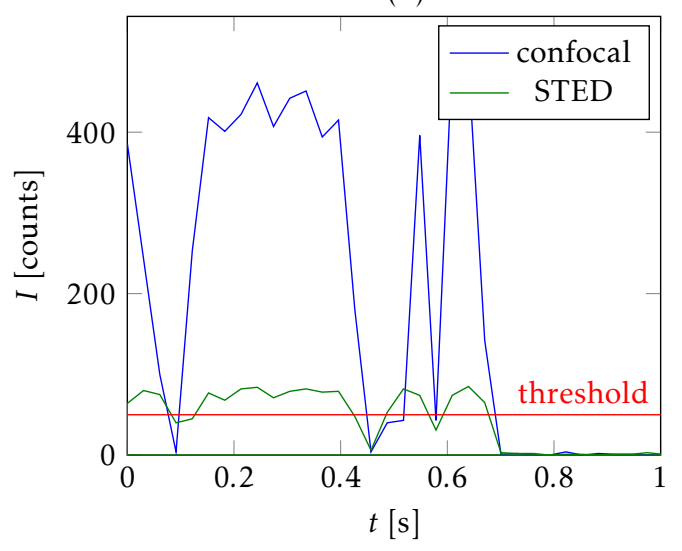

(c)

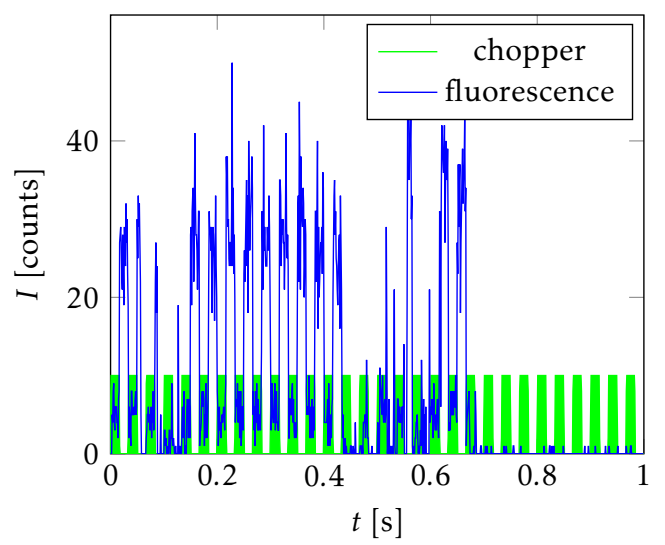

(b)

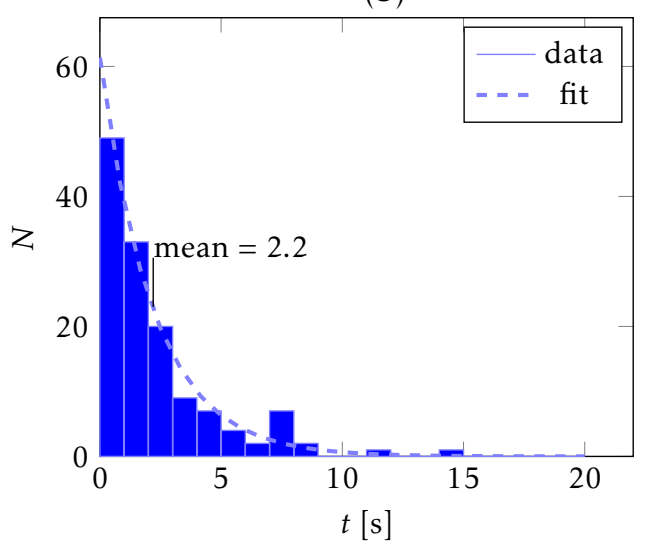

(d)

Figure A.6. (a): Fluorescence time trace of a single Atto647N molecule. (b): Unseparated data of a single molecule STED bleaching measurement. The STED laser is blocked periodically by a chopper to probe the bleaching time with the excitation. (c): Separated data of (b). The data is separated into a STED and confocal part and averaged over the chopper period. The number of confocal signal data points above the threshold are taken as the bleaching time. (d): Histogram of the measured bleaching times (Measurement 0 of Figure A.7) 


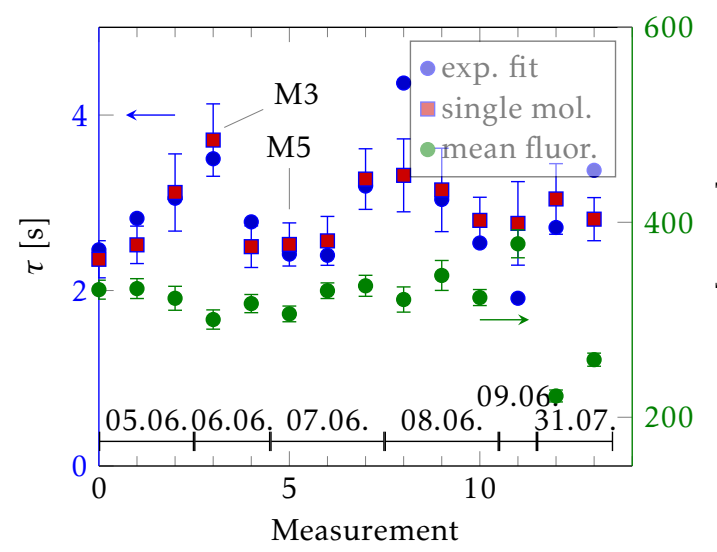

(a)

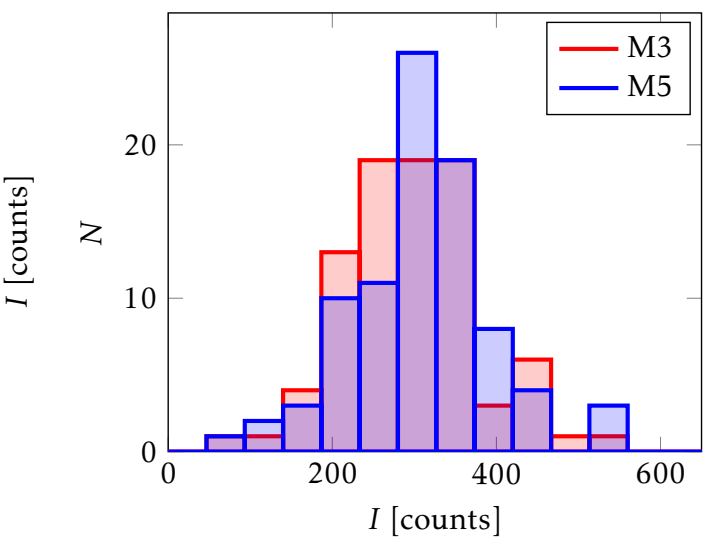

(b)

Figure A.7. (a): Development of the mean bleaching time (blue) during the acquisition of measurement series 1 (Figure 4.7) and mean single molecule fluorescence (green). The blue circles are the bleaching times calculated by fitting the summed fluorescence signal of all fluorophores (see Figure A.8), The red squares are the mean single molecule bleaching times. (b): Histogram of the mean fluorescence of single molecules, taken from measurement 3 (M3) and measurement 5 (M5). No correlation between mean fluorescence and bleaching rate was found.

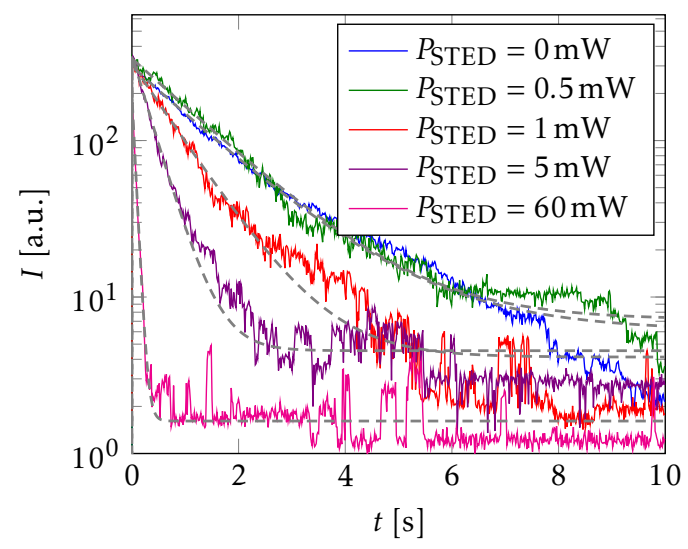

(a)

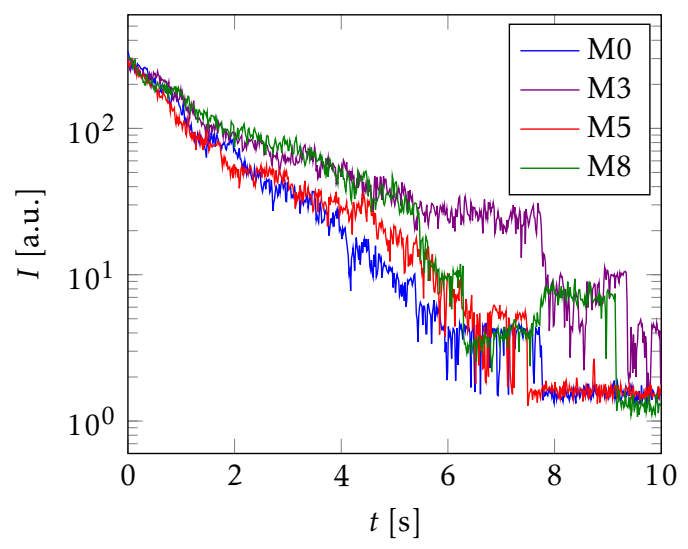

(b)

Figure A.8. (a): Summed single molecule fluorescence time traces and exponential fits (dashed lines) of measurement series 1 for selected STED powers. (b): Fluorescence decay of selected confocal measurements. The measurement indices corresponds to Figure A.7a. 


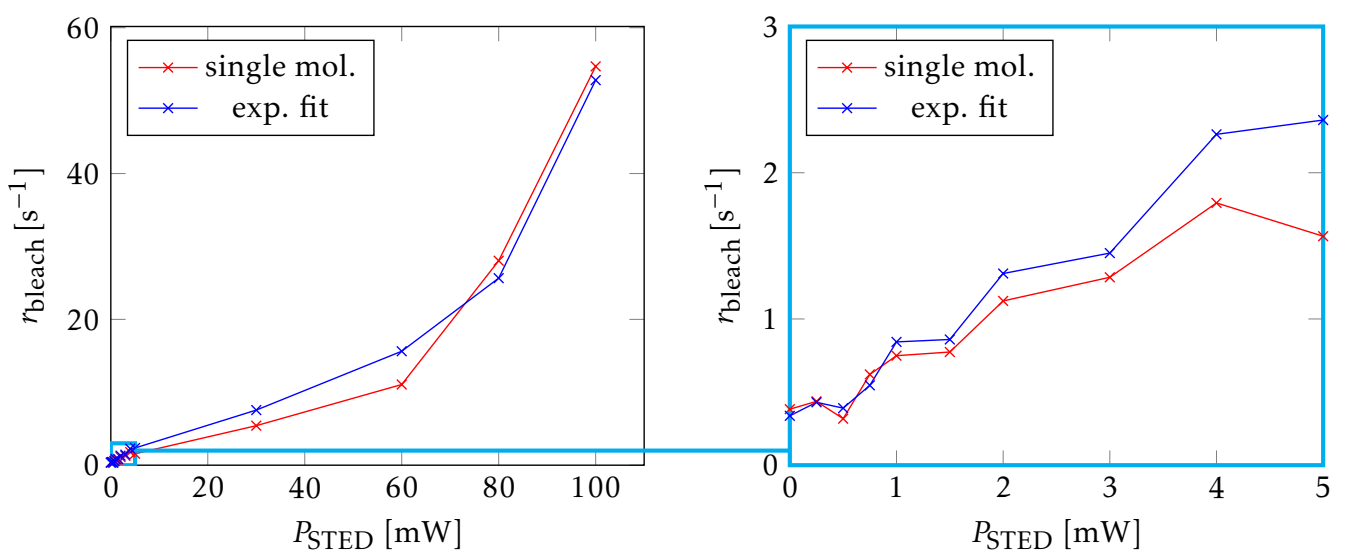

Figure A.9. Comparison between the two approaches to extract the bleaching time from the data. Taking the mean of the number of data points with fluorescence signal above the threshold (red) and fitting the summed data with an exponential (blue) yields very similar results.

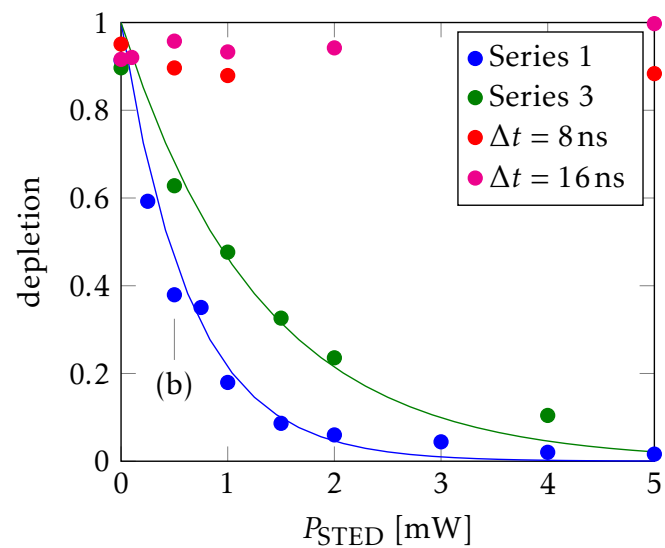

(a)

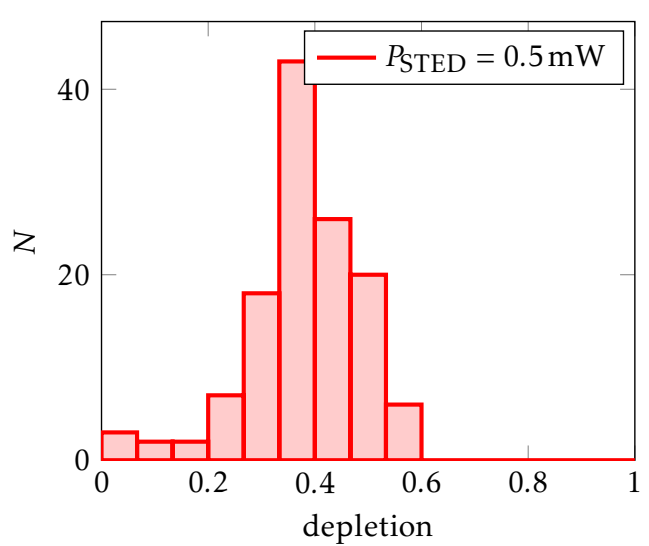

(b)

Figure A.10. Depletion by the STED laser. (a): Power dependency for various measurement series (see Figure 4.7). The solid lines are exponential decays for a saturation intensity of $2.5 \cdot 10^{5} \mathrm{~W} \mathrm{~cm}^{-2}$ (blue, $P_{\text {sat }}=0.45 \mathrm{~mW}$ ) and $5 \cdot 10^{5} \mathrm{~W} \mathrm{~cm}^{-2}$ (green, $P_{\text {sat }}=0.9 \mathrm{~mW}$ ). Please note that the intensity values are corrected for the laser power losses in the objective. (b): Histogram of the depletions of single molecules for the indicated data point. The broad distribution is due to abnormally behaving molecules (strong blinking: low depletion values, various states: high depletion values, see Figure A.11a.) 


\section{A. Appendix}

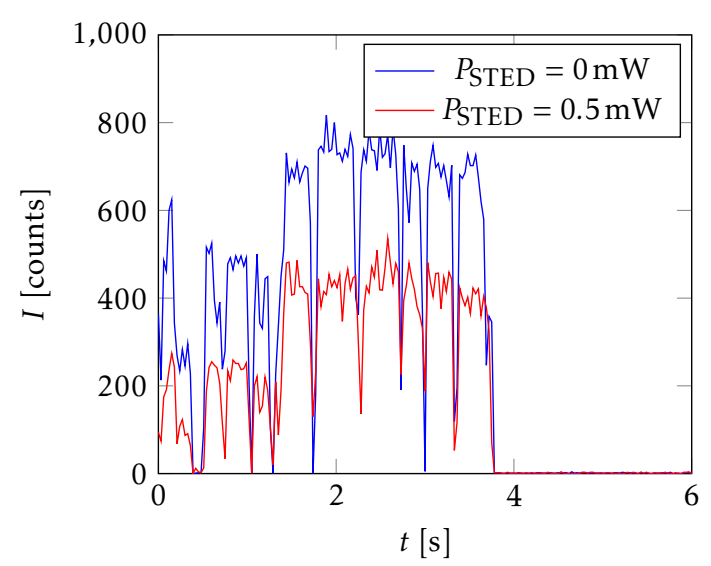

(a)

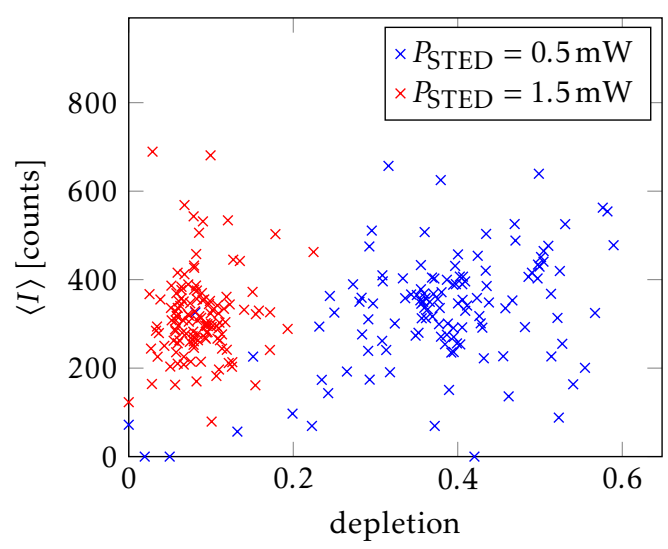

(b)

Figure A.11. Examples of varying single molecule behavior. (a): Confocal and STED signal of one fluorophore with inconsistent depletion behavior. (b): Mean fluorescence against depletion by the STED laser. No correlation is observable, thus the broad scattering of the depletion and brightness values are not due to varying laser power.

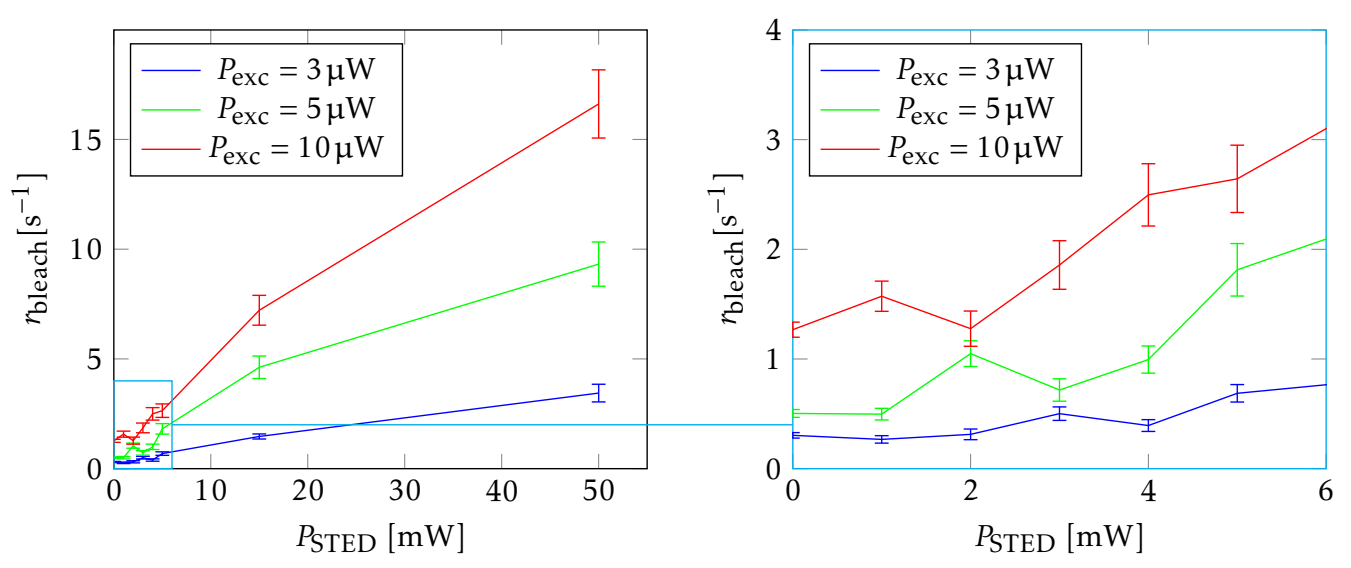

Figure A.12. For all STED powers the bleaching rate depends strongly on the excitation power. This indicates that the excitation to the $S_{1}$ state by the excitation laser is a necessary step in the bleaching pathway. 


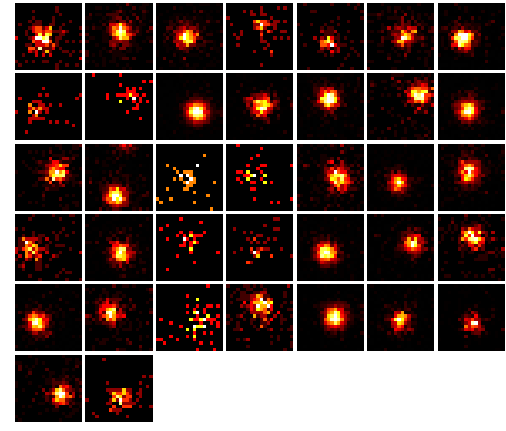

(a)

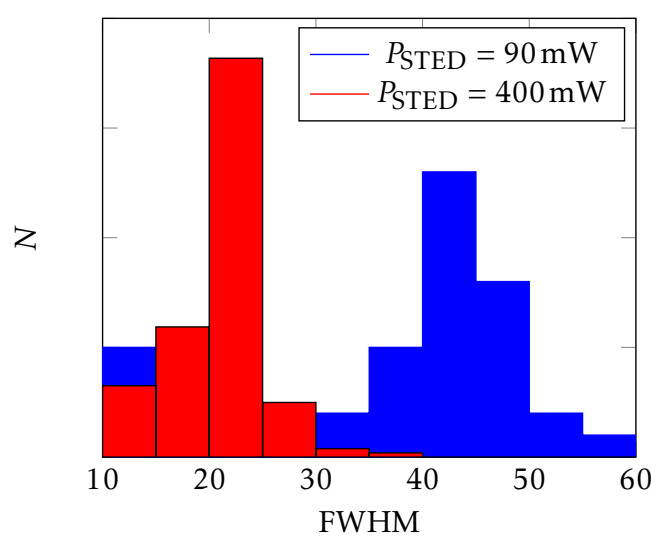

(c)

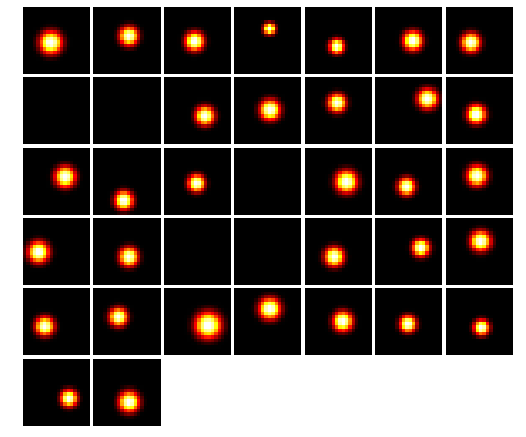

(b)

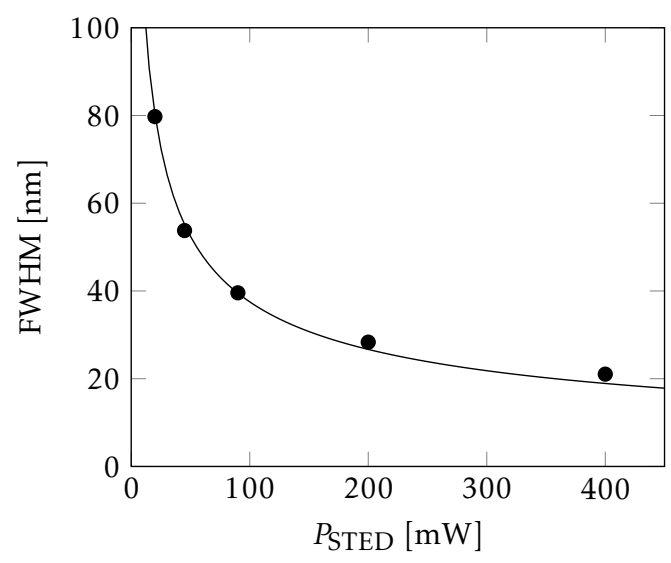

(d)

Figure A.13. Resolution measured on single molecules. (a) shows the normalized images of single molecules, measured at $P_{\text {STED }}=90 \mathrm{~mW}$ (image size: $200 \times 200 \mathrm{~nm}^{2}$ ). The data is fitted with a 2D-Gaussian (b). The fit values for the FWHM are shown in (c). The mean FWHM (after removing failed fits) for different scan STED powers is shown in (d). Straight line: Fit with equation (1.5) 


\section{A.4. Nanobodies}

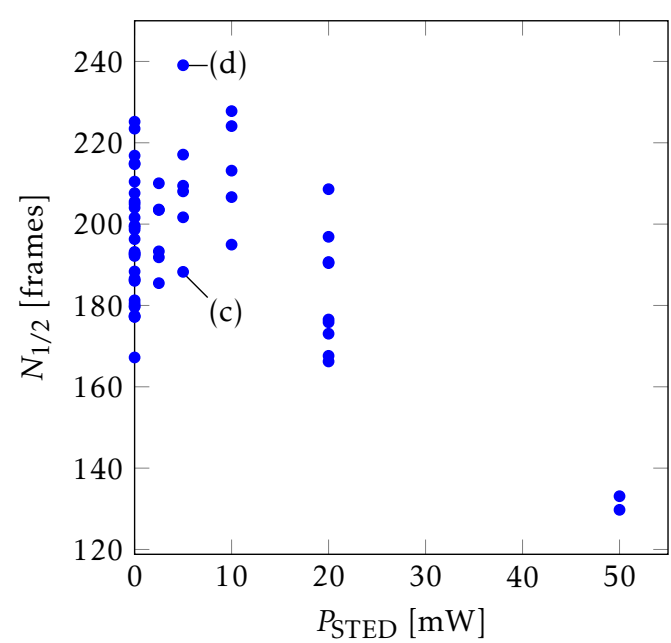

(a)

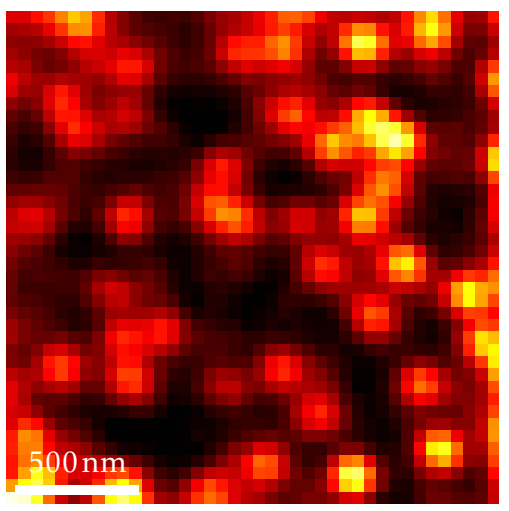

(c) $N_{1 / 2}=239$

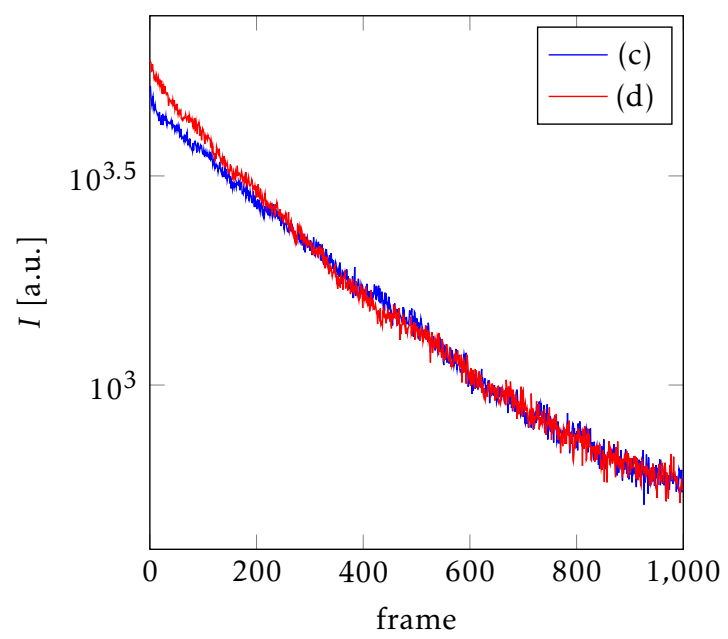

(b)

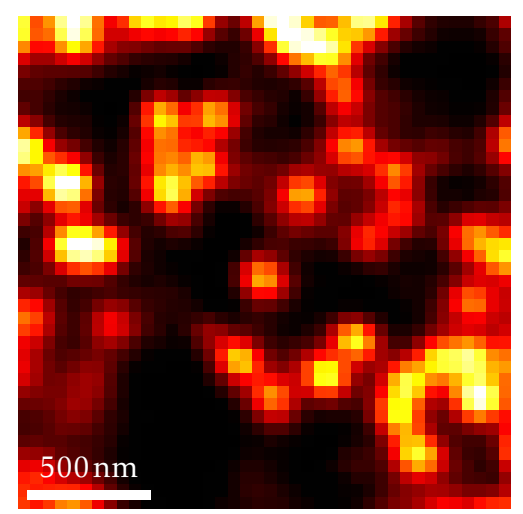

(d) $N_{1 / 2}=188$

Figure A.14. Imaging with low STED power can reduce the bleaching rate. Here, the bleaching times of the individual measurement positions are plotted (a). In contrast, the graph in Figure 4.13 shows the bleaching times acquired by summing the signal of all measurements and exponential fit. Although all measurement parameters are the same, the bleaching rate scatters strongly. This is caused by slightly different focus positions or the heterogeneity of the sample. (b) shows the fluorescence decay of the indicated data points, (c) and (d) are the respective images. To avoid a systematic influence of the bleaching data through defocus, it was alternated between STED and confocal measurements. 


\section{A.5. Published Articles}

\section{First author:}

Göttfert F, Wurm CA, Mueller V, Berning S, Cordes VC, Honigmann A, Hell SW. Coaligned dual-channel STED nanoscopy and molecular diffusion analysis at $20 \mathrm{~nm}$ resolution. Biophysical Journal. 2013;105(1):L01-L03.

\section{Collaborations:}

Chapochnikov NM, Takago H, Huang CH, Pangršič T, Khimich D, Neef J, Auge E, Göttfert F, Hell SW, Wichmann C, Wolf F, Moser T. Uniquantal release through a dynamic fusion pore is a candidate mechanism of hair cell exocytosis. Neuron. 2014;83(6):1389-1403.

D'Este E, Kamin D, Göttfert F, El-Hady A, Hell SW. STED nanoscopy reveals the ubiquity of subcortical cytoskeleton periodicity in living neurons. Cell Reports. 2015;10(8):1246-1251.

Jung S, Oshima-Takago T, Chakrabarti R, Wong AB, Jing Z, Yamanbaeva G, Picher MM, Wojcik SM, Göttfert F, Predoehl F, Micheli K, Hell SW, Schoch S, Strenzke N, Wichmann $\mathrm{C}$, Moser T. Rab3-interacting molecules $2 \alpha$ and $2 \beta$ promote the abundance of voltage-gated $\mathrm{Ca}_{V} 1.3 \mathrm{Ca}^{2+}$ channels at hair cell active zones. Proceedings of the National Academy of Sciences of the United States of America. 2015;112(24):E3141-E3149.

Kolmakov K, Wurm CA, Meineke DN, Göttfert F, Boyarskiy VP, Belov VN, Hell SW. Polar red-emitting rhodamine dyes with reactive groups: synthesis, photophysical properties, and twocolor STED nanoscopy applications. Chemistry A European Journal. 2014;20(1):146-157.

Lukinavičius G, Reymond L, D’Este E, Masharina A, Göttfert F, Ta H, Güther A, Fournier M, Rizzo S, Waldmann H, Blaukopf C, Sommer C, Gerlich DW, Arndt HD, Hell SW, Johnsson K. Fluorogenic probes for live-cell imaging of the cytoskeleton. Nature Methods. 2014;11(7):731-733.

Milovanovic D, Honigmann A, Koike S, Göttfert F, Pähler G, Junius M, Müllar S, Diederichsen U, Janshoff A, Grubmüller H, Risselada HJ, Eggeling C, Hell SW, van den Bogaart G, Jahn R. Hydrophobic mismatch sorts SNARE proteins into distinct membrane domains. Nature Communications. 2015;6:5984.

Siebert M, Böhme MA, Driller JH, Babikir H, Mampell MM, Rey U, Ramesh N, Matkovic T, Holton N, Reddy-Alla S, Göttfert F, Kamin D, Quentin C, Klinedinst S, Andlauer TF, Hell SW, Collins CA, Wahl MC, Loll B, Sigrist SJ. A high affinity RIM-binding protein/Aplip1 interaction prevents the formation of ectopic axonal active zones. eLife. 2015;4:e06935.

Wong AB, Rutherford MA, Gabrielaitis M, Pangršič T, Göttfert F, Frank T, Michanski S, Hell S, Wolf F, Wichmann C, Moser T. Developmental refinement of hair cell synapses tightens the coupling of $\mathrm{Ca}^{2+}$ influx to exocytosis. The EMBO Journal. 2014;33(3):247-264.

Wurm CA, Kolmakov K, Göttfert F, Ta H, Bossi M, Schill H, Berning S, Jakobs S, Donnert G, Belov VN, Hell SW. Novel red fluorophores with superior performance in STED microscopy. Optical Nanoscopy. 2012;1(1):1-7.

Zuidscherwoude M, Göttfert F, Dunlock VME, Figdor CG, van den Bogaart G, van Spriel AB. The tetraspanin web revisited by super-resolution microscopy. Scientific Reports. 2015;5:12201. 



\section{Curriculum Vitae}

\section{Personal Information}

Name: $\quad$ Fabian Göttfert

Date of Birth:

14.12.1986 in Zürich

\section{Acacemic Career}

Oct. 2006 - Nov. 2011

Study of physics at the Technische Universität Dresden, Germany. Finished with degree "Diplom-Physiker".

Feb. 2010 - Jun. 2010 ERASMUS student at the University of Sheffield, England.

Oct. 2010 - Nov. 2011 Diploma thesis at the Helmholtz-Zentrum DresdenRossendorf on the charge carrier relaxation dynamics in graphene.

Mar. 2012 - Dec. 2015 PhD student at the Max Planck Institute for Biophysical Chemistry, Göttingen, Germany. 



\section{Acknowledgments}

At this point, I would like to thank everybody who has made my time as $\mathrm{PhD}$ student such a successful and enjoyable period.

First and foremost Stefan Hell, for his brilliant ideas, helpful discussions, motivation and the possibility to attain my $\mathrm{PhD}$ in such a great environment during this interesting time. I would like to thank Erwin Neher and Sarah Köster for being members in my thesis committee and their help and advice during and beyond the meetings.

I owe a big Thank You to all the proof readers, it is their doing that the word count outnumbers the mistakes in this thesis. Namely to Jaydev Jethwa, whose help is invaluable also when encountering problems with anything from $50 \Omega$ resistors to laser cavities; to Jan Keller-Findeisen, who is always available for a good discussion when it includes at least one formula; and to my father, for his tireless re-reading and advice.

I would like to thank all my collaborators. It was their hard work that made the two-color setup such a success. I have learned not only a lot about biology, but also 101 ways to improve the usability of a STED microscope. First of all Christian Wurm. He introduced me to the practical aspects of fluorescence imaging, always knew what went wrong in the sample preparations, and his dedication to the project was a main drive on the way to publish the two-color setup. Then Elisa D'Este, who is extremely good at preparing and imaging neurons, organizing, lifting the office spirit and being a good friend. Volker Cordes and Tino Pleiner, who are responsible for the amazing nuclear pore samples (way more samples than shown here). I would like to point out the people in the listed publications who have directly worked on the setup, namely Alf Honigmann, Christian Wurm, Dragomir Milovanovic, Elisa D’Este, Gražvydas Lukinavičius, Jakob Neef, Malou Zuidscherwoude, Mark Rutherford, Mathias Böhme, SangYong Jung and Veronika Müller.

I am very grateful to Sebastian Berning, he did an excellent job in teaching me how to build a STED microscope. Most of the design decisions in the two-color setup are based on his work and advice. To Volker Westphal, for his great technical advice and helpful discussions. To Marco Roose and Björn Thiel, who have helped a lot with tackling computer and programming related issues. Due to Marco's administration, I never had to fear data loss or long-lasting network failures. Without Björn's help and advice, my graphical user interface would probably not have happened.

I thank all the people who kept things running smoothly. Our TAs, especially Ellen Rothermel, for preparing great samples on short notice and ensuring a steady supply of labeled antibodies. Rainer Pick and the optics, electronics and mechanical workshops for their reliable and prompt help and service. Our secretaries and the GGNB/PBCS office, for always helping out with all kinds of cryptic forms and applications.

Last but not least, I thank my family for their continuous support and motivation. 